H. Umemura

Nagoya Math. J.

Vol. 144 (1996), 59-135

\title{
DIFFERENTIAL GALOIS THEORY OF INFINITE DIMENSION
}

\author{
HIROSHI UMEMURA
}

\section{CONTENTS}

Introduction $\quad 59$

§1. Lie-Ritt functor $\quad 62$

$\S 2 . \quad$ Lie algebra of a Lie-Ritt functor $\quad 78$

$\S 3$. Construction of $\mathscr{K}$ and $\mathscr{L} \quad 92$

§4. Functors $\mathscr{F}_{\mathscr{L} / \mathcal{K}}$ and $\mathfrak{F}_{\mathscr{L} / \mathcal{K}} \quad 105$

$\S 5$. Infinitesimal Galois group $\quad 120$

References 134

\section{Introduction}

This paper is the second part of our work on differential Galois theory as we promised in [U3]. Differential Galois theory has a long history since Lie tried to apply the idea of Abel and Galois to differential equations in the 19th century (cf. [U3], Introduction). When we consider Galois theory of differential equation, we have to separate the finite dimensional theory from the infinite dimensional theory. As Kolchin theory shows, the first is constructed on a rigorous foundation. The latter, however, seems inachieved despite of several important contributions of Drach, Vessiot,.... We propose in this paper a differential Galois theory of infinite dimension in a rigorous and transparent framework. We explain the idea of the classical authors by one of the simplest examples and point out the problems. Let us consider an ordinary differential equation

$$
F\left(y, y^{\prime}, \ldots, y^{(n-1)}\right)=y^{(n)},
$$

where $F$ is a polynomial in $y, y^{\prime}, \ldots, y^{(n-1)}$ and its coefficients are meromorphic on a domain of $\mathbf{C}$. The derivation is taken with respect to the variable $x$ on $\mathbf{C}$. There are two procedures.

Received September 12, 1994. 
(i) Linearization process. They replace the differential (1) by the partial linear differential equation

(2) $\left(\partial / \partial x+y^{\prime} \partial / \partial y+y^{(2)} \partial / \partial y^{\prime}+\cdots+F\left(y, y^{\prime}, \ldots, y^{(n-1)}\right) \partial / \partial^{(n-1)}\right) Y=0$

satisfied by a first integral $Y$ of (1).

(ii) Galois theory of a partial linear differential equation. They seek Galois theory of a set of independent solutions $Y_{1}, Y_{2}, \ldots, Y_{n}$ of (2).

There are problems in each procedure. Let us try to formulate the idea in a more precise language. First of all, as in Galois theory of an algebraic equation, the Galois group should be attached not to a differential equation but to a field extension that a particular solution of the differential equation defines. So we must clarify the differential base field and the particular solution. Let $K$ be a diffrential field of meromorphic functions over a domain of $\mathbf{C}$ such that the coefficients of the polynomial $F\left(y, y^{\prime}, \ldots, y^{(n-1)}\right)$ are in $K$. Namely $K$ is the base field of the differential equation (1). We choose a solution $y$ of (1) once for all and study a differential field extension $K\left(y, y^{\prime}, \ldots, y^{(n-1)}\right)$ of $K$.

PROBlem 1. Starting from the ordinary base field $K$ and the solution $y$ of (1), if we try to pass from ordinary to partial, it is not clear at all how to choose a base field for the partial linear differential equation (2).

Leaving the Problem 1 aside, we take a set of independent first integrals (= solutions of (2)) $Y_{1}, Y_{2}, \ldots, Y_{n}$ in the linearlization process.

PROBlem 2. There is no canonical choice of the set of independent first integrals $Y_{1}, Y_{2}, \ldots, Y_{n}$

Even if the Problem 1 is settled so that we have a canonical base field $\mathscr{K}$ for the partial linear differential equation (2), the differential field extension $\mathscr{K}<Y_{1}$, $\left.Y_{2}, \ldots, Y_{n}\right\rangle$ depends on the choice of the set of first integrals $Y_{1}, Y_{2}, \ldots, Y_{n}$, i.e. a different choice gives a completely different partial differential field extension of the base field $\mathscr{K}$. Here we denote by $\mathscr{K}\left\langle Y_{1}, Y_{2}, \ldots, Y_{n}\right\rangle$ the partial differential field generated by $Y_{1}, Y_{2}, \ldots, Y_{n}$ over $\mathscr{K}$.

In most of works, the authors simply say that Galois theory of an ordinary differential equation is equivalent to Galois theory of a partial linear differential equation and they limit themselves to the second procedure (e.g. [D], [V1] and [P]). 
PROBlem 3. In the second procedure of developing Galois theory of partial linear differential equation, there are obscurities due to the lack of language.

For example Vessiot [V1] introduced an automorphic system, which is, roughly speaking, a principal homogeneous space of a Lie pseudogroup. Since a Lie pseudogroup is not a group, it requires a precision.

Inspired by a work of Vessiot [V2] in 1946, we overcome the Problems 1, 2 in the linearization process. Our setting is general. We consider an ordinary differential field extension $L / K$ of characteristic 0 . We canonically construct a partial differential field extention $\mathscr{L} / \mathscr{K}$ (cf. $\S 3$ ). To avoid the Problem 3 , we apply the method of [U3] to the partial differential field extension $\mathscr{L} / \mathscr{K}$ in $\S 4$ and 5 . We interpret a Lie pseudogroup as a formal group of infinite dimension or as a group functor on a category of rings (See $\S 1$ ). The idea of understanding a Lie pseudogroup as a formal group seems to go back to Ritt [R] of 1950 .

Let $L / K$ be an ordinary differential field of characteristic 0 such that $L$ is finitely generated over $K$ as an abstract field. We attach to $L / K$ the Galois group

$$
\text { Inf-gal }(L / K) \text {, }
$$

which is a formal group with coefficients in $L$ (Lie-Ritt functor on the category of $L$-algebras), in general of infinite dimension, where we regard the differential field $L$ as an abstract field.

Here are the main properties of the Galois group.

(i) If $M$ is a differential intermediate field of $L / K$, there exists a canonical surjective morphism Inf-gal $(L / K) \rightarrow$ Inf-gal $(M / K)$ (Theorem 5.14).

(ii) If $L / K$ is a strongly normal extension with Galois group $G$, then the differential Galois group $\operatorname{Inf}-\operatorname{gal}(L / K)$ is the formal completion $\hat{G}$ of $G$ (Theorem (5.15)).

(iii) If $L$ is finite algebraic over $K$, then $\operatorname{Inf-gal}(L / K)=0$ (Corollary (5.22)).

(iv) If $L$ is generated by constants over $K$, then $\mathbf{I n f}-\operatorname{gal}(L / K)=0$ (Corollary (5.22)).

\section{Index of notations}

$N(A)$

$\Gamma_{n}(A)$
The set of all nilpotent elements of a ring $A$

The group of all infinitesimal coordinate transformations of $n$-variables with coefficients in $A$ (Definition (1.1)) 
$\Gamma_{n R}$

$(\operatorname{Alg} / R)$

(Grp)

$A\{S\}$

$K\langle S\rangle$

$(\mathrm{Vec} / k)$

$(F \bmod / R)$

$\Theta_{n k}$

$L^{\#}$

$\mathscr{K}_{L / K}=\mathscr{K}$

$\mathscr{L}_{L / K}=\mathscr{L}$

$\mathscr{F}_{\mathscr{L} / \mathcal{H}}(A)$

$\mathfrak{F}_{\mathscr{L} / \mathcal{K}}(A)$

The Lie-Ritt functor of all infinitesimal coordinate transformations of $n$-variables on the category (Alg/R) (Definition (1.8))

The category of $R$-algebras

The category of groups

Differential algebra generated by a set $S$ over a differential ring $R$

Differential field generated by a set $S$ over a differential field $K$

The category of $k$-vector spaces

The category of free $R$-modules

The $k$-vector space of formal vector fields (Lemma (2.3))

Differential field $\left(L^{\natural},\left\{\partial / \partial u_{1}, \partial / \partial u_{2}, \ldots, \partial / \partial u_{n}\right\}\right)(\S 3)$

Differential subfield of $L^{\#}[[t]]\left[t^{-1}\right]$ generated by $L^{\#}$ and $i(K)(\S 3)$

Differential subfield of $L^{\#}[[t]]\left[t^{-1}\right]$ generated by $i(K)$ and $\mathscr{K}_{L / K}(\S 3)$

The set of all infinitesimal deformations with coefficients in an $L^{\natural}$-algebra $A$ of the canonical morphism $\mathscr{L} \rightarrow L^{\natural}[[w$, $t]]\left[t^{-1}\right](\S 4)$

The set of all infinitesimal deformations with coefficients in an $\mathscr{L}^{\natural}$-algebra $A$ of the universal Taylor morphism $\mathscr{L} \rightarrow$ $\mathscr{L}^{\natural}[[w, T]](\S 4)$

Inf-diff- $\operatorname{bir}_{K} L(A)$ The group of all continuous differential $\mathscr{K} \hat{\otimes}_{L^{\sharp}} A[[w]]$ automorphisms of $\mathscr{L} \widehat{\otimes}_{L^{\#}} A[[w]]$ that are congruent to the identity modulo $\mathscr{L} \bar{\bigotimes}_{L^{\sharp}} N[[w]](\S 5)$

Inf-gal $(L / K) \quad$ Infinitesimal differential Galois group (Definition (5.13))

\section{\$1. Lie-Ritt functor}

In this paper all the algebras, except for Lie algebras and Hopf algebras, are commutative $\mathbf{Q}$-algebras. For a commutative algebra $A$, we denote by $N(A)$ the set of all nilpotent elements of $A$ :

$$
N(A)=\left\{x \in A \mid x^{n}=0 \text { for some positive integer } n \text { depending on } x\right\} .
$$

So $N(A)$ is an ideal of $A$. 
Definition (1.1). For an algebra $A$, we set

$$
\begin{gathered}
\boldsymbol{\Gamma}_{n}(A)=\left\{\Phi=\left(\varphi_{1}, \varphi_{2}, \ldots, \varphi_{n}\right) \mid \varphi_{i}=\varphi_{i}(x) \in A\left[\left[x_{1}, x_{2}, \ldots, x_{n}\right]\right] \text { for } 1 \leq i \leq n\right. \\
\text { such that } \left.\Phi \equiv\left(x_{1}, x_{2}, \ldots, x_{n}\right) \bmod N(A)\right\}
\end{gathered}
$$

Here we mean by $\Phi \equiv\left(x_{1}, x_{2}, \ldots, x_{n}\right) \bmod N(A)$ that

$$
x_{\imath}-\varphi_{i}\left(x_{1}, x_{2}, \ldots, x_{n}\right) \in N(A)\left[\left[x_{1}, x_{2}, \ldots, x_{n}\right]\right]
$$

for $1 \leq i \leq n$. So geometrically $\Phi=\left(\varphi_{1}, \varphi_{2}, \ldots, \varphi_{n}\right)$ is a formal coordinate transformation of $n$-variables with coefficients in $A$ that is congruent to the identity modulo nilpotent elements. In other words $\Phi=\left(\varphi_{1}, \varphi_{2}, \ldots, \varphi_{n}\right)$ is an infinitesimal formal coordinate transformation of $n$-variables with coefficients in $A$.

Lemma (1.2). Let $\Psi=\left(\phi_{1}, \phi_{2}, \ldots, \phi_{n}\right)$ be another element of $\boldsymbol{\Gamma}_{n}(A)$. Then the composite

$$
\Psi \circ \Phi=\left(\phi_{1}\left(\varphi_{1}, \varphi_{2}, \ldots, \varphi_{n}\right), \phi_{2}\left(\varphi_{1}, \varphi_{2}, \ldots, \varphi_{n}\right), \ldots, \phi_{n}\left(\varphi_{1}, \varphi_{2}, \ldots, \varphi_{n}\right)\right)
$$

is well-determined in $\boldsymbol{\Gamma}_{n}(A)$.

Proof. We may assume for simplicity $n=1$. Let $\Phi=(\varphi(x))$ with

$$
\varphi(x)=a_{0}+a_{1} x+a_{2} x^{2}+\cdots
$$

and $\Psi=(\phi(x))$

$$
\psi(x)=b_{0}+b_{1} x+b_{2} x^{2}+\cdots
$$

Then

$$
\begin{aligned}
\varphi \circ \varphi=b_{0} & +b_{1}\left(a_{0}+a_{1} x+a_{2} x^{2}+\cdots\right) \\
& +b_{2}\left(a_{0}+a_{1} x+a_{2} x^{2}+\cdots\right)^{2} \\
& +\cdots \\
=b_{0} & +b_{1} a_{0}+b_{2} a_{0}^{2}+\cdots \\
& +\left(b_{1} a_{1}+2 b_{2} a_{0} a_{1}+3 b_{3} a_{0}^{2} a_{1}+4 b_{4} a_{0}^{3} a_{1}+\cdots\right) x+\cdots
\end{aligned}
$$

which is a formal power series in $\boldsymbol{\Gamma}_{1}(A)$ since $a_{0}$ is nilpotent.

Lemma (1.3). If we set $\operatorname{Id}=\left(x_{1}, x_{2}, \ldots, x_{n}\right)$, then $\operatorname{Id} \circ \Phi=\Phi \circ \mathrm{Id}=\Phi$.

Proof. The assertion follows the definition. 
Lemma (1.4). For $\Phi, \Psi, \Theta \in \Gamma_{n}(A)$, we have

$$
(\Phi \circ \Psi) \circ \Theta=\Phi \circ(\Psi \circ \Theta) \text {. }
$$

Namely the composition law ${ }^{\circ}$ is associative.

Proof. The element $\Phi \in \boldsymbol{\Gamma}_{n}(A)$ defines an $A$-algebra endomorphism

$$
\Phi^{*}: A\left[\left[x_{1}, x_{2}, \ldots, x_{n}\right]\right] \rightarrow A\left[\left[x_{1}, x_{2}, \ldots, x_{n}\right]\right] \quad\left(x_{i} \mapsto \varphi\left(x_{i}\right)\right)
$$

continuous with respect to the $\left(x_{1}, x_{2}, \ldots, x_{n}\right)$-adic topology. We can recover $\Phi$ from $\Phi^{*}$. Similarly the elements $\Psi, \Theta$, defines an $A$-algebra endomorphisms

$$
\Psi^{*}, \Theta^{*}: A\left[\left[x_{1}, x_{2}, \ldots, x_{n}\right]\right] \rightarrow A\left[\left[x_{1}, x_{2}, \ldots, x_{n}\right]\right] .
$$

The lemma follows from the identity

$$
\Theta^{*}\left(\circ \Psi^{*} \circ \Phi^{*}\right)=\left(\Theta^{*} \circ \Psi^{*}\right) \circ \Phi^{*},
$$

which is the associativity law for composition of maps between sets.

Lemma (1.5). For $\Phi \in \boldsymbol{\Gamma}_{n}(A)$, there exists $\Psi \in \boldsymbol{\Gamma}_{n}(A)$ such that

$$
\Psi \circ \Phi=\mathrm{Id} .
$$

Namely every element of $\boldsymbol{\Gamma}_{n}(A)$ has a left inverse.

Proof. We may assume that $n=1$ for simplicity. Let $\Phi=(\varphi(x))$ with

$$
\varphi(x)=a_{0}+a_{1} x+a_{2} x^{2}+\cdots \in \Gamma_{n}(A) .
$$

Since the composition law $\circ$ is associative by Lemma (1.4), it is sufficient to show that

$$
\left(x-a_{0}\right) \circ \varphi(x)=a_{1} x+a_{2} x^{2}+\cdots
$$

has a left inverse. So we may assume that $a_{0}$ is equal to 0 . Now as is well-known, since $a_{0}=0$ and $a_{1}$ is invertible in $A$, the series $\varphi(x)$ has the (left) inverse $\phi(x)$ in $A[[x]]$. We denote the classes of $\varphi(x), \phi(x) \bmod N(A)[[x]]$ respectively by $\bar{\varphi}(x), \bar{\psi}(x)$. Since the quotient ring

$$
A[[x]] / N(A)[[x]]
$$

is isomorphic to

$$
(A / N(A))[[x]]
$$


we can regard

$$
\bar{\varphi}(x), \bar{\phi}(x) \in(A / N(A))[[x]] .
$$

Then it follows from

$$
\psi^{\circ} \varphi(x)=\varphi^{\circ} \psi(x)=x \text { and } \bar{\varphi}(x) \equiv x \text { in }(A / N(A))[[x]]
$$

that $\bar{\phi} \circ \bar{\varphi}(x)=\bar{\varphi} \circ \bar{\phi}(x)=x$ in $(A / N(A))[[x]]$ and hence $\bar{\phi}(x)=x$ in $(A / N(A))[[x]]$. Namely $\phi(x) \equiv x \bmod N(A)[[x]]$. Hence $\phi(x)$ is in $\boldsymbol{\Gamma}_{1}(A)$ so that $\varphi(x)$ has a left inverse $\phi(x)$.

Proposition (1.6). The set $\boldsymbol{\Gamma}_{n}(A)$ forms a group with respect to the composition law ${ }^{\circ}$.

Proof. If a composition law satisfies the following conditions, then it is a group law:

(i) It is associative;

(ii) There exists a left identity;

(iii) Every element has a left inverse.

This fact is well known (cf. Chap. I, $\$ 2$, p. 9 of [L] for example). Proposition now follows from Lemmas (1.3), (1.4) and (1.5).

COROLlary (1.6.1). We have a canonical anti-isomorphism of groups:

$\Gamma_{n}(A) \stackrel{\sim}{\rightarrow}\left\{\Phi \in \operatorname{Aut}_{A} A\left[\left[x_{1}, x_{2}, \ldots, x_{n}\right]\right] \mid \Phi\right.$ is continuous with respest to the $\left(x_{1}, x_{2}, \ldots, x_{n}\right)$-adic topology and the reduction of

$\Phi$ modulo $N(A)$ is the identity map of $\left.A / N(A)\left[\left[x_{1}, x_{2}, \ldots, x_{n}\right]\right]\right\}$.

Proof. We use the notation in the proof of Lemma (1.4). For $\Phi \in \boldsymbol{\Gamma}_{n}(A)$, we can find by Proposition (1.6) $\Lambda \in \boldsymbol{\Gamma}_{n}(A)$ such that we have

$$
\Lambda \circ \Phi=\Phi \circ \Lambda=\mathrm{Id}
$$

so that we have

$$
\Phi^{*} \circ \Lambda^{*}=\Lambda^{*} \circ \Phi^{*}=\mathrm{Id}
$$

Hence

$$
\Phi^{*}: A\left[\left[x_{1}, x_{2}, \ldots, x_{n}\right]\right] \rightarrow A\left[\left[x_{1}, x_{2}, \ldots, x_{n}\right]\right]
$$

is an $A$-automorphism of $A\left[\left[x_{1}, x_{2}, \ldots, x_{n}\right]\right]$. Since ${ }^{*}$ is contravariant, the map- 
ping $\Phi \mapsto \Phi^{*}$ gives the canonical anti-isomorphism of the groups.

Corollary (1.6.1) gives a coordinate free description of the group $\boldsymbol{\Gamma}_{n}(A)$. Let $R$ be a $\mathbf{Q}$-algebra. The category of $R$-algebra will be denoted by $(A l g / R)$. So we can regard $A \mapsto \Gamma_{n}(A)$ as a group functor. To make the reference algebra $R$ clear, we add a subscript $R$ :

$$
\Gamma_{n R}:(A l g / R) \rightarrow(G r p), \quad A \mapsto \Gamma_{n}(A)
$$

for an $R$-algebra $A$.

Definition (1.7). We call the group functor $\boldsymbol{\Gamma}_{n R}$ the Lie-Ritt functor of all infinitesimal coordinate transformations of $n$-variables defined over $R$.

For an $R$-algebra $A$ the formal power series ring

$$
A\left[\left[x_{1}, x_{2}, \ldots, x_{n}\right]\right]
$$

is a differential ring with respect to the derivations

$$
\left\{\partial / \partial x_{1}, \partial / \partial x_{2}, \ldots, \partial / \partial x_{n}\right\}
$$

We consider the formal jet space. Let

$$
A\left[\left[x_{1}, x_{2}, \ldots, x_{n}\right]\right]\left\{y_{1}, y_{2}, \ldots, y_{n}\right\}
$$

be a differential polynomial ring with coefficients in the differential algebra

$$
A\left[\left[x_{1}, x_{2}, \ldots, x_{n}\right]\right] .
$$

So as an abstract ring

$$
A\left[\left[x_{1}, x_{2}, \ldots, x_{n}\right]\right]\left\{y_{1}, y_{2}, \ldots, y_{n}\right\}=A\left[\left[x_{1}, x_{2}, \ldots, x_{n}\right]\right]\left[y_{i}^{\left(j_{1}, j_{2}, \ldots, j_{n}\right)}\right] \underset{\substack{1 \leq i \leq n \\\left(j_{1}, j_{2}, \ldots, j_{n}\right) \in \mathbf{N}^{n}}}{ }
$$

where the $y_{i}^{\left(j_{1}, j_{2}, \ldots, j_{n}\right)}$ are indeterminates over the ring $A\left[\left[x_{1}, x_{2}, \ldots, x_{n}\right]\right]$. The derivations

$$
\left\{\partial / \partial x_{1}, \partial / \partial x_{2}, \ldots, \partial / \partial x_{n}\right\}
$$

of $A\left[\left[x_{1}, x_{2}, \ldots, x_{n}\right]\right]$ are extended to

$$
A\left[\left[x_{1}, x_{2}, \ldots, x_{n}\right]\right]\left\{y_{1}, y_{2}, \ldots, y_{n}\right\}
$$

by 


$$
\frac{\partial}{\partial x_{l}} y_{i}^{\left(j_{1}, j_{2}, \ldots, j_{n}\right)}=y_{l}^{\left(j_{1}, j_{2}, \ldots, j_{l-1}, j_{l}+1, j_{l+1}, \ldots, j_{n}\right)}
$$

for

$$
1 \leq i, l \leq n,\left(j_{1}, j_{2}, \ldots, j_{n}\right) \in \mathbf{N}^{n} .
$$

We denote the formal complection

$$
A\left[\left[x_{1}, x_{2}, \ldots, x_{n}\right]\right]\left[\left[y_{\imath}^{\left(j_{1}, j_{2}, \ldots, j_{n}\right)}\right]\right]_{1 \leq \imath \leq n}\left(j_{1}, j_{2}, \ldots, j_{n}\right) \in \mathbf{N}^{n}
$$

by

$$
A\left[\left[x_{1}, x_{2}, \ldots, x_{n}\right]\right]\left\{\left\{y_{1}, y_{2}, \ldots, y_{n}\right\}\right\}
$$

which is a differential algebra with derivations

$$
\left\{\partial / \partial x_{1}, \partial / \partial x_{2}, \ldots, \partial / \partial x_{n}\right\}
$$

Let $I$ be a differential ideal of the formal power series ring

$$
R\left[\left[x_{1}, x_{2}, \ldots, x_{n}\right]\right]\left\{\left\{y_{1}, y_{2}, \ldots, y_{n}\right\}\right\} .
$$

We denote by $I_{A}$ a differential ideal [I] of

$$
A\left[\left[x_{1}, x_{2}, \ldots, x_{n}\right]\right]\left\{\left\{y_{1}, y_{2}, \ldots, y_{n}\right\}\right\}
$$

generated by $I$ for an $R$-algebra $A$.

Definition (1.8). A Lie-Ritt functor defined over a $\mathbf{Q}$-algebra $R$ is a group functor on the category $(\mathrm{Alg} / \mathrm{R})$ isomorphic to a group subfunctor $G$ of the group functor $\boldsymbol{\Gamma}_{n R}$ defined by a differential ideal of

$$
R\left[\left[x_{1}, x_{2}, \ldots, x_{n}\right]\right]\left\{\left\{y_{1}, y_{2}, \ldots, y_{n}\right\}\right\} .
$$

Namely there exists a differential ideal $I$ of

$$
R\left[\left[x_{1}, x_{2}, \ldots, x_{n}\right]\right]\left\{\left\{y_{1}, y_{2}, \ldots, y_{n}\right\}\right\}
$$

such that we have

$$
G(A)=\left\{\Phi \in \Gamma_{n}(A) \mid F(\Phi)=0 \text { for every } F \in I_{A}\right\} \text { for each } R \text {-algebra } A .
$$

Here in the equation $F(\Phi)=0$ we substitute

$$
\frac{\partial^{j} \varphi_{l}}{\partial x_{1}^{j_{1}} \partial x_{2}^{j_{2}} \cdots \partial x_{n}^{j_{n}}} \quad\left(j=\sum_{l=1}^{n} j_{l}\right)
$$


for

$$
y_{i}^{\left(j_{1}, j_{2}, \ldots, j_{n}\right)}
$$

Let us see some examples.

Example (1.9). In the following examples (i), (ii), (iii), $n=1$ and $R=\mathbf{Q}$.

(i) Let us denote the additive group $N(A)$ by $\hat{\mathbf{G}}_{a}(A)$ for a $\mathbf{Q}$-algebra $A$ so that

$$
\tilde{\mathbf{G}}_{a \mathbf{Q}}(A l g / \mathbf{Q}) \rightarrow(G r p), A \mapsto \hat{\mathbf{G}}_{a}(A)
$$

for a $\mathbf{Q}$-algebra $A$, is a group functor. We consider a subset

$$
G_{+}(A)=\left\{a_{0}+x \mid a_{0} \in N(A)\right\}
$$

of $\boldsymbol{\Gamma}_{1}(A)$ is a subgroup for a $\mathbf{Q}$-algebra $A$. Hence

$$
A \mapsto G_{+}(A)
$$

is a group subfunctor of the group functor $\Gamma_{1 \mathbf{Q}}$. We have a functorial isomorphism

$$
\hat{\mathbf{G}}_{a}(A) \rightarrow G_{+}(A)
$$

that sends $a_{0}$ to $a_{0}+x$ for $a_{0} \in \hat{\mathbf{G}}_{a}(A)$. Thus the group functor $\hat{\mathbf{G}}_{a \mathbf{Q}}$ is isomorphic to the group functor $G_{+}$. Now we choose a differential ideal $I=\left[y^{\prime}-1\right]$ of $\mathbf{Q}[[x]]\{\{y\}\}$. Then the set

$$
\left\{\varphi(x) \in \Gamma_{1}(A) \mid \varphi^{\prime}(x)-1=0\right\}
$$

coincides with the subgroup $G_{+}(A)$ for a $\mathbf{Q}$-algebra $A$ so that the group functor $\overline{\mathbf{G}}_{a \mathbf{Q}}(A)$ is a Lie-Ritt functor. For any $\mathbf{Q}$-algebra $R$ the restriction of the Lie-Ritt functor $\overline{\mathbf{G}}_{a \mathbf{Q}}(A)$ to the subcategory $(A l g / R)$ will be denoted by $\overline{\mathbf{G}}_{a R}$.

(ii) Let us set

$$
\hat{\mathbf{G}}_{m \mathbf{Q}}(A)=\{a \in A \mid a-1 \in N(A)\}
$$

so that $\hat{\mathbf{G}}_{m \mathbf{Q}}(A)$ is a group by the multiplication in the ring $A$. We get a group functor

$$
\hat{\mathbf{G}}_{m \mathbf{Q}}:(A l g / \mathbf{Q}) \rightarrow(G r p), A \mapsto \hat{\mathbf{G}}_{m \mathbf{Q}}(A)
$$

for a $\mathbf{Q}$-algebra $A$. Let us consider a subset

$$
\left\{a_{1} x \in \Gamma_{1}(A)\right\}
$$

of $\boldsymbol{\Gamma}_{1}(A)$ for a $\mathbf{Q}$-algebra $A$. Then the subset is in fact a subgroup of $\boldsymbol{\Gamma}_{1}(A)$. 
Hence we get a group subfunctor $G_{\times}$of the group functor $\boldsymbol{\Gamma}_{1}$ :

$$
G_{\times}:(A \lg / \mathbf{Q}) \rightarrow(G r p), \quad A \mapsto\left\{a_{1} x \in \Gamma_{1}(A)\right\}
$$

for a $\mathbf{Q}$-algebra $A$. Moreover there exists a functorial isomorphism of groups

$$
\hat{\mathbf{G}}_{m \mathbf{Q}}(A) \rightarrow G_{\times}(A) \quad a \mapsto a x .
$$

So the group functor $\hat{\mathbf{G}}_{m \mathbf{Q}}$ is isomorphic to $G_{\times}$. We consider now a differential ideal $\left[x y^{\prime}-y\right]$ of $\mathbf{Q}[[x]]\{\{y\}\}$. Then the subset $\left\{\varphi(x) \in \boldsymbol{\Gamma}_{1}(A) \mid x \varphi^{\prime}(x)-\right.$ $\varphi(x)=0\}$ coincides with the subgroup $G_{\times}(A)$. Hence $\overline{\mathbf{G}}_{m \mathbf{Q}}$ is a Lie-Ritt functor defined over $\mathbf{Q}$. Similarly as for the group functor $\hat{\mathbf{G}}_{a \mathbf{Q}}$, the restriction of the group functor $\widehat{\mathbf{G}}_{m \mathbf{Q}}$ to the subcategory $(\mathrm{Alg} / R)$ for a $\mathbf{Q}$-algebra $R$ will be denoted by $\overline{\mathbf{G}}_{m R}$.

(iii) We consider a subgroup

$$
G(A)=\left\{\frac{a x+b}{c x+b} \mid\left(\begin{array}{ll}
a & b \\
c & d
\end{array}\right)-I_{2} \in M_{2}(N(A)), a d-b c=1\right\} \subset \Gamma_{1}(A)
$$

for a $\mathbf{Q}$-algebra $A$. We notice here that $d$ is invertible in $A$ and hence

$$
\frac{a x+b}{c x+d}=d^{-1}(a x+b)\left(1-d^{-1} c x+\left(d^{-1} c x\right)^{2}-\cdots\right)
$$

is a formal power series in $x$. As is well-known a transformation

$$
x \mapsto \frac{a x+b}{c x+d}
$$

is characterized by the Schwarzian derivative $\{y ; x\}$ (Let us recall

$$
\left.\{y ; x\}=\left(\frac{d^{3} y}{d x^{3}}\right) /\left(\frac{d y}{d x}\right)-\frac{3}{2}\left[\left(\frac{d^{2} y}{d x^{2}}\right) /\left(\frac{d y}{d x}\right)\right]^{2}\right) \text {. }
$$

Namely $G(A)=\left\{\varphi(x) \in \Gamma_{1}(A) \mid\{\varphi(x) ; x\}=0\right\}$. So $G$ is a Lie-Ritt functor.

(iv) Let us take now $R=\mathbf{Q}$ as above but $n$ arbitrary. We set

$$
G(A)=\left\{\Phi=\left(\varphi_{1}, \varphi_{2}, \ldots, \varphi_{n}\right) \in \Gamma_{n}(A) \mid \text { The Jacobian } \frac{D\left(\varphi_{1}, \varphi_{2}, \ldots, \varphi_{n}\right)}{D\left(x_{1}, x_{2}, \ldots, x_{n}\right)}=1\right\}
$$

for a $\mathbf{Q}$-algebra $A$. Then $G$ is a group subfunctor of $\boldsymbol{\Gamma}_{n}$ defined by the differential equation

$$
\frac{D\left(\varphi_{1}, \varphi_{2}, \ldots, \varphi_{n}\right)}{D\left(x_{1}, x_{2}, \ldots, x_{n}\right)}=1
$$


So $G$ is a Lie-Ritt functor.

The Examples (1.9.i), (1.9.ii), (1.9.iii) are finite dimensional. Namely the dimension of the Lie algebra of the each group functor, which will be defined in $\S 2$, is finite dimensional. The Examples (i) and (ii) are of dimension 1. The Example (iii) depends on 3 parameters. In Example (iv), however the solution of the differential equation

$$
\frac{D\left(\varphi_{1}, \varphi_{2}, \ldots, \varphi_{n}\right)}{D\left(x_{1}, x_{2}, \ldots, x_{n}\right)}=1
$$

depends on infinitely many parameters if $n \geq 2$.

Definition 1.10. We define a morphism of Lie-Ritt functors over a ring as a morphism of group functors.

For example, we have for a $\mathbf{Q}$-algebra $A$ a functorial morphism

$$
\overline{\mathbf{G}}_{a \mathbf{Q}}(A) \rightarrow \overline{\mathbf{G}}_{m \mathbf{Q}}(A), \quad a \mapsto \exp a
$$

of groups. So there exists a morphism

$$
\exp : \overline{\mathbf{G}}_{a \mathbf{Q}} \rightarrow \hat{\mathbf{G}}_{m \mathbf{Q}}
$$

of Lie-Ritt functors. Similarly for a $\mathbf{Q}$-algebra $A$, we have a functorial morphism

$$
\hat{\mathbf{G}}_{m \mathbf{Q}}(A) \rightarrow \hat{\mathbf{G}}_{a \mathbf{Q}}(A), \quad a \mapsto \log a
$$

of groups, which gives us a morphism

$$
\log : \overline{\mathbf{G}}_{m \mathbf{Q}} \rightarrow \hat{\mathbf{G}}_{a \mathbf{Q}}
$$

of group functors. Since $\exp { }^{\circ} \log =\mathrm{Id}, \log { }^{\circ} \exp =\mathrm{Id}$, the Lie-Ritt functors $\widehat{\mathbf{G}}_{a \mathbf{Q}}$ and $\hat{\mathbf{G}}_{m \mathbf{Q}}$ are mutually isomorphic. Customarily the equivalence of Lie pseudogroup is defined by using prolongations. Our definition seems new and quite natural.

A formal group yields an example of a Lie-Ritt functor. Let us recall the definition of a formal group.

Definition (1.11). A formal group over a ring $R$ is an $n$-tuple $F=\left(f_{i}\right)$ of formal power series

$$
f_{i}(u, v) \in R\left[\left[u_{1}, u_{2}, \ldots, u_{n}, v_{1}, v_{2}, \ldots, v_{n}\right]\right]=R[[u, v]]
$$


such that

(1) $F(u, 0)=u$ and $F(0, v)=v$,

(2) $F(u, F(v, w))=F(F(u, v), w)$.

We know that there exists

$$
\Theta(u)=\left(\theta_{1}(u), \theta_{2}(u), \ldots, \theta_{n}(u)\right) \in R\left[\left[u_{1}, u_{2}, \ldots, u_{2}\right]\right]^{n}
$$

such that $\Theta(0)=0$ and such that

$$
F(u, \Theta(u))=F(\Theta(u), u)=0
$$

(cf. Serre [S] LG Chap. $4, \S 6$ ). Let $A$ be an $R$-algebra. For

$$
a=\left(a_{1}, a_{2}, \ldots, a_{n}\right), b=\left(b_{1}, b_{2}, \ldots, b_{n}\right) \in N(A)^{\mathrm{n}}
$$

we define the product of $a$ and $b$ by

$$
a b=F(a, b) .
$$

Then $N(A)^{n}$ is a group by this group law. The unit element is given by 0 and $\Theta(a)$ is the inverse $a^{-1}$ of $a$. We denote this group by $N(A)_{F}^{n}$. Hence the formal group $F$ defines a group functor

$$
\mathbf{F}:(A l g / R) \rightarrow(G r p), \quad A \mapsto N(A)_{F}^{n} .
$$

The group functor $\mathbf{F}$ is a Lie-Ritt functor. In fact let $I$ be the differential ideal in $R[[x]]\{\{y\}\}$ generated by

$$
\frac{\partial F(y, \Theta(x))}{\partial x_{i}}=\left.\left(\sum_{i=1}^{n} \frac{\partial F(u, v)}{\partial u_{i}} \frac{\partial y_{l}}{\partial x_{i}}+\sum_{i=1}^{n} \frac{\partial F(u, v)}{\partial v_{i}} \frac{\partial \theta_{l}}{\partial x_{i}}\right)\right|_{(u, v)=(y, \Theta(x))}, \quad 1 \leq i \leq n .
$$

We show that the group functor $\mathbf{F}$ is isomorphic to the Lie-Ritt functor defined by the ideal $I$. Let $A$ be an $R$-algebra and

$$
\Phi(x)=\left(\varphi_{1}, \varphi_{2}, \ldots, \varphi_{n}\right) \in \Gamma_{n R}(A) .
$$

Assume that

$$
\frac{\partial F(y, \Theta(x))}{\partial x_{i}}=\left.\left(\sum_{i=1}^{n} \frac{\partial F(u, v)}{\partial u_{i}} \frac{\partial y_{l}}{\partial x_{i}}+\sum_{i=1}^{n} \frac{\partial F(u, v)}{\partial v_{i}} \frac{\partial \theta_{l}}{\partial x_{i}}\right)\right|_{(u, v)=(\Phi(x), \boldsymbol{\theta}(x))}=0
$$

for $1 \leq i \leq n$. The system (1.12) of equations is equivalent to

$$
\partial M / \partial x_{i}=0 \text { for } 1 \leq i \leq n,
$$

where we set $M=F(\Phi(x), \Theta(x))$. So the system (1.12) of the differential equa- 
tions is satisfied if and only if $M=F(\Phi(x), \Theta(x)) \in A[[x]]^{n}$ is constant, or

$$
F(\Phi(x), \Theta(x))=\left(a_{1}, a_{2}, \ldots, a_{n}\right)
$$

with $a_{\imath} \in A$ for $1 \leq i \leq n$. If we pass to $A / N(A)$, then $\Phi(x)$ is equal to

$$
0 \in(A / N(A))[[x]]^{n}
$$

and hence by (1.13) $\left(a_{1}, a_{2}, \ldots, a_{n}\right)$ is congruent to 0 modulo $N(A)$. Namely the $a_{\imath} \in A$ are in fact in $N(A)$. We have thus shown that the following conditions are equivalent:

(1) $H(\Phi(x))=0$ for every $H \in I$;

(2) $\partial M / \partial x_{i}=0$ for $1 \leq i \leq n$;

(3) $F(\Phi(x), \Theta(x))=a$ with $a \in N(A)^{n}$.

Now if $F(\Phi(x), \Theta(x))=a$, then

$$
\Phi(x)=F(a, x) .
$$

Therefore we proved that if we set

$$
G(A)=\left\{\Phi \in \Gamma_{n A} \mid H(\Phi(x))=0 \text { for every } H \in I\right\},
$$

then the mapping

$$
\pi: G(A) \rightarrow N(A)^{n}=N(A)_{F}^{n}, \quad \Phi(x) \mapsto F(\Phi(x), \Theta(x))=a
$$

is bijective. This mapping $\pi$ is an isomorphism of groups. In fact, let now

$$
\Phi(x), \Psi(x) \in G(A)
$$

so that we can find by (1.14)

$$
a, b \in N(A)^{n}
$$

such that

$$
\Phi(x)=F(a, x), \Psi(x)=F(b, x) .
$$

So since $F$ is a formal group, we have

$$
\Phi \circ \Psi(x)=F(a, F(b, x))=F(F(a, b), x) .
$$

Therefore

$$
\pi(\Phi \circ \Psi)=F(a, b)
$$

by the above equivalence of conditions and $\pi$ is a group homomorphism. Since the group isomorphism $\pi$ is functorial in $A$, the group functor $G$ is isomorphic to the 
group functor $\mathbf{F}$ associated with the formal group $F$.

Let

$$
\begin{aligned}
& F(u, v) \in R\left[\left[u_{1}, u_{2}, \ldots, u_{m}, v_{1}, v_{2}, \ldots, v_{m}\right]\right]^{m}, \\
& G(y, z) \in R\left[\left[y_{1}, y_{2}, \ldots, y_{n}, z_{1}, z_{2}, \ldots, z_{n}\right]\right]^{n}
\end{aligned}
$$

be formal groups. A morphism $f: F \rightarrow G$ of formal groups is an $n$-tuple

$$
f=\left(f_{1}, f_{2}, \ldots, f_{n}\right) \in R\left[\left[u_{1}, u_{2}, \ldots, u_{m}\right]\right]^{n}
$$

such that $f_{\imath}(0)=0$ for $1 \leq i \leq n$ and such that

$$
f(F(u, v))=G(f(u), f(v)) .
$$

The morphism of formal groups

$$
f: F \rightarrow G
$$

induces a morphism of group functors

$$
\mathbf{f}: \mathbf{F} \rightarrow \mathbf{G} \text {. }
$$

Therefore we get a functor of the category of formal groups over $R$ to the categpry of Lie-Ritt functors over $R$.

Lemma (1.15). The functor $F \mapsto \mathbf{F}$ is fully faithful. Namely we have

$$
\operatorname{Hom}_{\text {formal group }}(F, G) \simeq \operatorname{Hom}_{\text {Lie-Ritt functor }}(\mathbf{F}, \mathbf{G})
$$

for any two formal groups over $R$.

Proof. We denote by 0 the subfunctor of $N^{d}$ attaching the point $((0,0, \ldots$, 0 ) of $N(A)$ to every $R$-algebra $A$. It is sufficient to show that a morphism of functors

$$
f: N^{m} \rightarrow N^{n}
$$

that maps $0 \in N^{m}$ to $0 \in N^{n}$ is defined by an $n$-tuple

$$
\left(f_{1}, f_{2}, \ldots, f_{n}\right) \in R\left[\left[u_{1}, u_{2}, \ldots, u_{m}\right]\right]^{n}
$$

such that $f_{i}(0)=0$ for $1 \leq i \leq n$ and such that we have

$$
f(a)=\left(f_{1}(a), f_{2}(a), \ldots, f_{n}(a)\right)
$$

for

$$
a=\left(a_{1}, a_{2}, \ldots, a_{m}\right) \in N(A)^{m}
$$


Let us prove this under the assumption $m=n=1$, the general case being treated in the similar way. Let us set

$$
A_{r}=R[x] / x^{r} \text { for } r \in \mathbf{N}
$$

Then the map

$$
f_{r}=f\left(A_{r}\right): N\left(A_{r}\right) \rightarrow N\left(A_{r}\right)
$$

gives

$$
a_{0}^{(r)}, a_{1}^{(r)}, \ldots, a_{r-1}^{(r)}, \in R
$$

such that

$$
f_{r}(\bar{x})=\sum_{i=1}^{n} a_{i}^{(r)} \bar{x}^{2}
$$

$\bar{x}$ being the class of $x$ in $A_{r}$. Since the diagram

$$
\begin{aligned}
& N\left(A_{r+1}\right) \stackrel{f_{r+1}}{\longrightarrow} N\left(A_{r+1}\right) \\
& N\left(A_{r}\right) \quad \underset{f_{r}}{\longrightarrow} N\left(A_{r}\right)
\end{aligned}
$$

is commutative, the vertical arrows being the canonical morphism, we have

$$
a_{i}^{(r)}=a_{i}^{(r+1)} \text { for } 0 \leq i \leq r-1 .
$$

So $a_{i}^{(r)} \in R$ is independent of $r$ and hence we denote it by $a_{i} \in R$ for $i \in \mathbf{N}$. We find the $a_{\imath} \in R$ for $i \in \mathbf{N}$ such that we have in $A_{r}$

$$
f_{r}(\bar{x})=\sum_{i=0}^{r-1} a_{i} \bar{x}^{i} \quad \text { for every } r \in \mathbf{N} .
$$

Let now $A$ be an $R$-algebra and $a \in N(A)$ so that we have $a^{r}=0$ for an integer $r \geq 0$. So there exists an $R$-morphism

$$
\varphi: R[x] / x^{r} \rightarrow A, \quad x \mapsto a .
$$

Since the diagram

is commutative, we have

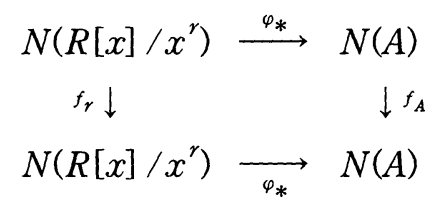

$$
f_{A}(a)=\sum_{i=0}^{\infty} a_{i} a^{i}
$$


So the morphism

$$
f: N \rightarrow N
$$

of functors is defined by the power series

$$
\sum_{i=0}^{\infty} a_{i} x^{i}
$$

Since $f(0)=0$, we have $a_{0}=0$ and the lemma is proved.

We can regard a Lie-Ritt functor as a formal group of infinite variables. Let us take for example $\boldsymbol{\Gamma}_{n R}$. We assume for simplicity $n=1$. Let $A$ be an $R$-algebra and let

$$
\phi=a_{0}+\left(1+a_{1}\right) x+a_{2} x^{2}+\cdots
$$

and

$$
\phi=b_{0}+\left(1+b_{1}\right) x+b_{2} x^{2}+\cdots
$$

be power series in $\boldsymbol{\Gamma}_{1 R}(A)$ so that $a_{\imath}, b_{i}$ are in $N(A)$. Then

$$
\begin{aligned}
\psi^{\circ} \phi= & b_{0}+\left(1+b_{1}\right) a_{0}+b_{2} x_{0}^{2}+\cdots \\
& +\left(1+b_{1}+a_{1}+b_{1} a_{1}+2 b_{2} a_{0}\left(1+a_{1}\right)+3 b_{3} a_{0}^{2}\left(1+a_{1}\right)+\cdots\right) x \\
& +\cdots .
\end{aligned}
$$

So we can find formal power series

$$
f_{\imath}(u, v) \in R[[u, v]]=R\left[\left[u_{0}, u_{1}, u_{2}, \ldots, v_{0}, v_{1}, v_{2}, \ldots\right]\right]
$$

for $i \in \mathbf{N}$ such that

$$
\phi \circ \phi=f_{0}(b, a)+\left(1+f_{1}(b, a)\right) x+f_{2}(b, a) x^{2}+\cdots
$$

with

$$
a=\left(a_{0}, a_{1}, a_{2}, \ldots\right), \quad b=\left(b_{0}, b_{1}, b_{2}, \ldots\right)
$$

Then

$$
F(u, v)=\left(f_{0}(u, v), f_{1}(u, v), f_{2}(u, v), \ldots\right)
$$

is a formal group of infinite variables $u_{0}, u_{1}, u_{2}, \ldots$ Namely the set $F(u, v)$ of the power series satisfies the conditions (1), (2) of Definition (1.11). Now we consider a group subfunctor $G$ of $\Gamma_{1 R}$ and a differential ideal $I$ of $A[[x]]\{y\}$ in Definition (1.8) with $n=1$. Let

$$
\phi=u_{0}+\left(1+u_{1}\right) x+u_{2} x^{2}+\cdots
$$


be an element of $R\left[\left[u_{0}, u_{1}, u_{2}, \ldots\right]\right][[x]]$. Setting the coefficient of the power series in $x$ equal to 0 , we can show that the condition $F(\phi(x))=0$ for an element

$$
F\left(y, y^{(1)}, y^{(2)}, \ldots\right) \in I \subset R[[x]]\{y\}
$$

is equivalent to a set of power series relations among $u_{0}, u_{1}, u_{2}, \ldots$ with coefficients in $R$. So there exists a family of power series $h_{\alpha}\left(u_{0}, u_{1}, u_{2}, \ldots\right) \in R\left[\left[u_{0}\right.\right.$, $\left.u_{1}, u_{2}, \ldots\right]$ indexed by an appropriate set $J$ satisfying the following condition. For every $R$-algebra $A$ and $\phi(x) \in A[[x]]$ with $\phi(x) \equiv x \bmod N(A)$, the following conditions are equivalent:

(1) $h_{\alpha}\left(u_{0}, u_{1}, u_{2}, \ldots\right)=0$ for every $\alpha \in J$;

(2) $\phi(x) \in G(A)$.

In other words, the ideal $\left(h_{\alpha}(u)\right)_{\alpha \in J} \subset R[[u]]=R\left[\left[u_{0}, u_{1}, u_{2}, \ldots\right]\right]$ defines a formal subgroup of the formal group $F(u, v)$. This argument works also for $n \geq 2$. So we have proved

PROPOSITION (1.17). Every Lie-Ritt functor over a ring $R$ is represented by a formal group scheme over $R$.

A formal group is equivalent to a complete commutative Hopf algebra. For example, the set (1.16) of power series defines on the power series ring

$$
R[[u]]=R\left[\left[u_{0}, u_{1}, u_{2}, \ldots\right]\right]
$$

a continuous comultiplication

$$
R[[u]] \rightarrow R[[u]] \widehat{\otimes}_{R} R[[u]]
$$

by sending $u_{i}$ to $f_{i}((u) \otimes 1,1 \otimes(u))$ so that $R[[u]]$ is a continuous commutative Hopf algebra over $R$ and the formal spectrum $\operatorname{Spf} \mathrm{R}[[u]]$ is the formal group $\boldsymbol{\Gamma}_{1 R}$. Here the power series ring $R[[u]]$ is a topological ring by $(u)$-adic topology and we take the completion of the tensor product

$$
R[[u]] \bigotimes_{R} R[[u]]
$$

with respect to the $((u) \otimes 1+1 \otimes(u))$-adic topology.

This point of view is close to the formulation of Ritt [R], which Weifeiler [W] and Nichols $[\mathrm{N}]$ later developed by introducing differential Hopf algebra. It seems that Ritt's trial of defining formal group originated from the infinite dimensional differential Galois theory. We explain now their idea for the simplest case of $\boldsymbol{\Gamma}_{1 \mathbf{C}}$ and compare with ours. Ritt considers general functions 


$$
\bar{\phi}(x)=x+\phi(x), \quad \bar{\phi}(x)=x+\phi(x)
$$

and their composite

$$
\bar{\phi}(\bar{\phi}(x))=x+\phi(x)+\phi(x+\phi(x))=x+\phi(x)+\phi(x)+\sum_{n=1}^{\infty} \frac{\phi^{(n)}(x)}{n !} \phi(x)^{n} .
$$

If we introduce a differential indeterminate $\phi$ over the differential ring $(\mathbf{C}[[x]]$, $d / d x)$ and if we define on the completion

$$
\mathbf{C}[[x]]\{\{\phi\}\}=\mathbf{C}[[x]]\left[\left[\phi, \phi^{(1)}, \phi^{(2)}, \ldots\right]\right]
$$

of the differential polynomial ring

$$
\mathbf{C}[[x]]\{\phi\}=\mathbf{C}[[x]]\left[\phi, \phi^{(1)}, \phi^{(2)}, \ldots\right]
$$

a continuous comultiplication

$$
\mathbf{C}[[x]]\{\{\phi\}\} \rightarrow \mathbf{C}[[x]]\{\{\phi\}\} \hat{\otimes}_{\mathbf{C}[x]]} \mathbf{C}[[x]]\{\{\phi\}\},
$$

which is a differential $\mathbf{C}[[x]]$-algebra homomorphism, by

$$
\phi \mapsto \phi \otimes 1+1 \otimes \phi+\sum_{n=1}^{\infty} \frac{1 \otimes \phi^{(n)}}{n !} \phi^{n} \otimes 1
$$

(so that in general

$$
\phi^{(n)} \mapsto\left(\phi \otimes 1+1 \otimes \phi+\sum_{n=1}^{\infty} \frac{1 \otimes \phi^{(n)}}{n !} \phi^{n} \otimes 1\right)^{(n)},
$$

for $n \in \mathbf{N}$, e.g.

$$
\begin{gathered}
\phi^{(1)} \mapsto\left(\phi \otimes 1+1 \otimes \phi+\sum_{n=1}^{\infty} \frac{1}{n !}\left\{\left(1 \otimes \phi^{(n+1)}\right)\left(\phi^{n} \otimes 1\right)\right.\right. \\
\left.\left.+\left(1 \otimes \phi^{(n)}\right)\left(n \phi^{n-1} \phi^{(1)} \otimes 1\right)\right\}\right),
\end{gathered}
$$

then $\mathbf{C}[[x]]\{\{\phi\}\}$ is a continuous commutative Hopf algebra, a formal $\mathbf{C}[[x]]\left[\frac{d}{d x}\right]$-group with $K=\mathbf{C}[[x]]$ and $k=\mathbf{C}$ in the terminology of Weisfeiler and Nichols (cf. [N] p. 971). Here the completion of the tensor product is taken with respect to the $((\phi) \otimes 1+1 \otimes(\phi))$-adic topology. In [N] $K$ is a field but in this example $K$ is a ring and the definition is easily extended to the ring case.

What is the relation between these two continuous Hopf algebras

$$
\mathbf{C}\left[\left[u_{0}, u_{1}, u_{2}, \ldots\right]\right] \text { and } \mathbf{C}[[x]]\left[\left[\phi, \phi^{(1)}, \phi^{(2)}, \ldots\right]\right] \text { ? }
$$


For every $\mathbf{C}$-algebra $A$ both

$$
\left\{f \in \operatorname{Hom}_{\mathbf{C}}(\mathbf{C}[[u]], A) \mid f\left(u_{\imath}\right) \in N(A) \text { for every } i \in \mathbf{N}\right\}
$$

and

$$
\begin{gathered}
\left\{f \in \text { Diff-hom }_{\mathbf{C}[\{x]\}}\left(\mathbf{C}[[x]]\left[\left[\phi, \phi^{(0)}, \phi^{(2)}\right]\right], A[[x]]\right) \mid f(\phi)=a_{0}+a_{1} x+a_{2} x^{2}\right. \\
\left.+\cdots \text { with } a_{i} \in N(A) \text { for all } i \in \mathbf{N}\right\}
\end{gathered}
$$

give the group $\boldsymbol{\Gamma}_{1 \mathbf{C}}(A)$ by definition. We can define a continuous $R$-derivation $\delta$ on $R[[u]]$ by

$$
\delta u_{i}=(i+1) u_{i+1} \quad \text { for } \quad i \in \mathbf{N} .
$$

So $\mathbf{C}[[u]]$ is a formal $\mathbf{C}\left[\frac{d}{d x}\right]$-group with $K=k=\mathbf{C}$ (loc. cit.) and there exists a continuous $\mathbf{C}[[x]]$-isomorphism

$$
\mathbf{C}[[x]] \widehat{\otimes}_{\mathbf{C}} \mathbf{C}\left[\left[u_{0}, u_{1}, u_{2}, \ldots\right]\right] \rightarrow \mathbf{C}[[x]]\left[\left[\phi, \phi^{(1)}, \phi^{(2)}, \ldots\right]\right]
$$

sending $u_{i}$ to $\frac{1}{i !} \phi^{(i)}$ of Hopf algebras with derivation. Here the tensor product is that of differential algebras (i.e. we define $\left.(a \otimes b)^{\prime}=a^{\prime} \otimes 1+1 \otimes b^{\prime}\right)$ and the completion is taken with respect to the $1 \otimes(u)$-adic topology. We believe that the continuous Hopf algebra $R[[u]]$ is more natural than $\mathbf{C}[[x]]\left[\left[\phi, \phi^{(1)}, \phi^{(2)}, \ldots\right]\right]$ for the following reasons.

(1) The Hopf algebra $R[[u]]$ is defined over $\mathbf{Z}$. However the Hopf algebra

$$
\mathbf{C}[[x]]\left[\left[\phi, \phi^{(1)}, \phi^{(2)}, \ldots\right]\right]
$$

is defined only over $\mathbf{Q}$.

(2) All the Lie-Ritt functors are usual formal groups without derivation (in general of infinite dimension).

(3) What we need in the general differential Galois theory is a formal group without derivation.

\section{\$2. Lie algebra of a Lie-Ritt functor}

For a reasonable group functor on a category of schemes, we can define its Lie algebra by a general theory developed in Exposé II of [S.G.A.D]. In particular a Lie-Ritt functor has its Lie algebra. It is convenient, however, to review how we define the Lie algebra of a Lie-Ritt functor in the frame work of [S.G.A.D.].

For a $k$-vector space $V$, the direct sum $k \oplus V$ has a structure of $k$-algebra if 
we define the product of elements of $V$ to be 0 . Namely we define the product of two elements of $k \oplus V$ by

$$
(a, u)(b, v)=(a b, a v+b u) \quad \text { for }(a, u),(b, v) \in k \oplus V .
$$

We denote the algebra $k \oplus V$ by $D(V)$. So we have a projection

$$
p: D(V)=k \oplus V \rightarrow k
$$

which is a $k$-algebra homomorphism. Let $G$ be a group functor on the category of $k$-algebras. Hence we have a homomorphism

$$
p_{*}: G(D(V)) \rightarrow G(k)
$$

of groups induced by the morphism $p$ of $k$-algebras. We denote the kernel of $p_{*}$ by $L_{G}(V)$. Therefore we get a functor

$$
L_{G}:(V e c / k) \rightarrow(S e t), \quad V \mapsto L_{G}(V),
$$

where $(\mathrm{Vec} / \mathrm{k})$ denotes the category of $k$-vector spaces. We set

$$
\text { Lie } G=L_{G}\left(V_{1}\right)
$$

for a 1 dimensional $k$-vector space $V_{1}$ so that we have

$$
\text { Lie } G=\operatorname{Ker} p_{*} \text {. }
$$

Here $p_{*}$ denotes the homomorphism of groups

$$
p_{*}: G\left(D\left(V_{1}\right)\right) \rightarrow G(k)
$$

induced by the projection $p: D\left(V_{1}\right) \rightarrow k$. We show that if $G$ satisfies a reasonable condition, Lie $G$ has a natural structure of Lie algebra. Usually the following notation is employed. Let $k[\varepsilon]\left(\varepsilon^{2}=0\right)$ be the ring of dual numbers over $k$ so that

$$
k[\varepsilon] \simeq k[x] /\left(x^{2}\right)
$$

and the ring $k[\varepsilon]$ is $k$-isomorphic to $D\left(V_{1}\right)$. Under this isomorphism the projection $p$ coincides with the $k$-homomorphism

$$
q: k[\varepsilon] \rightarrow k, \quad \varepsilon \mapsto 0
$$

of algebras. Hence

$$
\text { Lie } G=\operatorname{Ker} q_{*} \text {, }
$$

where 


$$
q_{*}: G(k[\varepsilon]) \rightarrow G(k)
$$

is the homomorphism of groups induced by $q: k[\varepsilon] \rightarrow k$. We refer the reader for a general argument to [S.G.A.D.] and just illustrate the defintion of the Lie algebra structure on Lie $G$ for a Lie-Ritt functor $G$. For this reason we restrict ourselves to the following group functors on the category $(\mathrm{Alg} / \mathrm{k}$ ) of $k$-algebras.

(1) Lie-Ritt functors. In particular the group functor $\boldsymbol{\Gamma}_{n k}$ of all infinitesimal coordinate transformations of $n$ variables.

(2) The general linear group $\mathbf{G L}(W)$ for a $k$-vector space $W$.

Since $\boldsymbol{\Gamma}_{n}(k)=1$ by definition, the morphism

$$
p_{*}: \boldsymbol{\Gamma}_{n k}(D(V)) \rightarrow \boldsymbol{\Gamma}_{n k}(k)
$$

is trivial so that we have

$$
L_{\boldsymbol{\Gamma}_{n k}}(V)=\operatorname{Ker} p_{*}=\boldsymbol{\Gamma}_{n k}(D(V)) .
$$

In particular we have

$$
\text { Lie } \boldsymbol{\Gamma}_{n k}=\boldsymbol{\Gamma}_{n k}\left(D\left(V_{1}\right)\right) \text {. }
$$

Hence we have

$$
L_{\boldsymbol{\Gamma}_{n k}}:(V e c / k) \rightarrow(S e t), \quad V \mapsto L_{\boldsymbol{\Gamma}_{n k}}(V)=\boldsymbol{\Gamma}_{n k}(D(V))
$$

Lemma (2.1). Let $V, W$ be two $k$-vector spaces. Then we have a canonical isomorphism

$$
L_{\boldsymbol{\Gamma}_{n k}}(V \oplus W) \simeq L_{\boldsymbol{\Gamma}_{n k}}(V) \times L_{\boldsymbol{\Gamma}_{n k}}(W) .
$$

In other words the functor

$$
L_{\boldsymbol{\Gamma}_{n k}}:(\mathrm{Vec} / k) \rightarrow(\mathrm{Set})
$$

transforms a product to a product.

Proof. Lemma follows from the following observation. Let

$$
\Phi=\left(\varphi_{1}, \varphi_{2}, \ldots, \varphi_{n}\right) \in D(V \oplus W)\left[\left[x_{1}, x_{2}, \ldots, x_{n}\right]\right]^{n} .
$$

Then $\Phi \in L_{\boldsymbol{\Gamma}_{n k}}(V \oplus W)$ if and only if every coefficients of $\varphi_{i}-x_{\imath}$ lies in $V \oplus W$ for $1 \leq i \leq n$.

COROllary (2.2). Lie $\boldsymbol{\Gamma}_{n}=L_{\boldsymbol{\Gamma}_{n}}\left(V_{1}\right)$ has a structure of an additive group. 
Proof. Since the functor

$$
L_{\boldsymbol{\Gamma}_{n k}}:(\mathrm{Vec} / k) \rightarrow(\mathrm{Set})
$$

transforms a product to a product by Lemma (2.1), the addition

$$
V_{1} \oplus V_{1} \rightarrow V_{1}
$$

which is a $k$-linear map, induces a map

$$
L_{\boldsymbol{\Gamma}_{n k}}\left(V_{1}\right) \times L_{\boldsymbol{\Gamma}_{n k}}\left(V_{1}\right) \simeq L_{\boldsymbol{\Gamma}_{n k}}\left(V_{1} \oplus V_{1}\right) \rightarrow L_{\boldsymbol{\Gamma}_{n k}}\left(V_{1}\right) .
$$

This map defines an additive group structure on Lie $L_{\boldsymbol{\Gamma}_{n k}}=\boldsymbol{\Gamma}_{n}\left(D\left(V_{1}\right)\right)$.

We have a multiplication

$$
m_{\lambda}: V_{1} \rightarrow V_{1}
$$

for $\lambda \in k$, which induces a map

$$
m_{\lambda_{*}}: L_{\boldsymbol{\Gamma}_{n k}}\left(V_{1}\right) \rightarrow L_{\boldsymbol{\Gamma}_{n k}}\left(V_{1}\right) .
$$

If we set

$$
\lambda . \Phi=m_{\lambda_{*}}(\Phi) \quad \text { for } \Phi \in L_{\boldsymbol{\Gamma}_{n k}}\left(V_{1}\right), \lambda \in k,
$$

then Lie $\boldsymbol{\Gamma}_{n}=L_{\boldsymbol{\Gamma}_{n k}}\left(V_{1}\right)=\boldsymbol{\Gamma}_{n k}\left(D\left(V_{1}\right)\right)$ is a $k$-vector space. Now we define a product on the $k$-vector space Lie $\boldsymbol{\Gamma}_{n}=L_{\boldsymbol{\Gamma}_{n k}}\left(V_{1}\right)$ such that Lie $\boldsymbol{\Gamma}_{n}$ is a $k$-Lie algebra.

Lemma (2.3). Let $\Theta_{n k}$ be the $k$-vector space

$$
\begin{gathered}
k\left[\left[x_{1}, x_{2}, \ldots, x_{n}\right]\right]^{n} \partial / \partial x_{1}+k\left[\left[x_{1}, x_{2}, \ldots, x_{n}\right]\right]^{n} \partial / \partial x_{2} \\
+\cdots+k\left[\left[x_{1}, x_{2}, \ldots, x_{n}\right]\right]^{n} \partial / \partial x_{n}
\end{gathered}
$$

of all formal vector fields. Then we have a canonical isomorphism

$$
D: \text { Lie } \Gamma_{n} \rightarrow \Theta_{n k}
$$

of $k$-vector spaces.

Proof. Let $V_{1}$ be a $k$-vector space of dimension 1 . Then the algebra $D\left(V_{1}\right)$ is $k$-isomorphic to the ring

$$
k[\varepsilon]\left(\varepsilon^{2}=0\right)
$$

of dual numbers. So

Lie $\boldsymbol{\Gamma}_{n k}=\boldsymbol{\Gamma}_{n k}\left(D\left(V_{1}\right)\right) \simeq \boldsymbol{\Gamma}_{n k}(k[\varepsilon])=\left\{\Phi=\left(\varphi_{1}, \varphi_{2}, \ldots, \varphi_{n}\right) \in k[\varepsilon]\left[\left[x_{1}, x_{2}, \ldots, x_{n}\right]\right]^{n}\right.$ 
$\varphi_{\imath}-x_{\imath} \in \varepsilon k\left[\left[x_{1}, x_{2}, \ldots, x_{n}\right]\right]$ for $\left.1 \leq i \leq n\right\}$.

Hence if

$$
\Phi=\left(\varphi_{1}, \varphi_{2}, \ldots, \varphi_{n}\right) \in \Gamma_{n k}(k[\varepsilon])
$$

then there exist

$$
\phi_{i} \in k\left[\left[x_{1}, x_{2}, \ldots, x_{n}\right]\right]
$$

such that

$$
\varphi_{i}=x_{i}+\varepsilon \psi_{i}
$$

for $1 \leq i \leq n$. Therefore if we define a map

$$
D: \boldsymbol{\Gamma}_{n k}(k[\varepsilon]) \rightarrow \Theta_{n k}
$$

by

$$
D(\Phi)=\phi_{1} \partial / \partial x_{1}+\phi_{2} \partial / \partial x_{2}+\cdots+\phi_{n} \partial / \partial x_{n},
$$

then $D$ is bijective. It follows from the definition of the $k$-vector space structure of Lie $\boldsymbol{\Gamma}_{n k}$ that this map $D$ is in fact $k$-linear.

The above argument allows us to prove the following result.

Lemma (2.4). Let

$$
G:(A l g / k) \rightarrow(G r p)
$$

be a group functor. If the functor

$$
L_{G}:(V e c / K) \rightarrow(S e t) \quad V \mapsto \operatorname{Ker} p_{*}
$$

transforms a product to a product, then Lie $G$ is a $k$-vector space.

In particular we can apply this argument to a Lie-Ritt functor to get the following

Corollary (2.5). If $G$ is a Lie-Ritt functor, then Lie $G$ is a k-vector space.

Proof. We may assume that $G$ is a group subfunctor of $\boldsymbol{\Gamma}_{n k}$ so that $G$ is defined by a differential ideal $I$ of

$$
k\left[\left[x_{1}, x_{2}, \ldots, x_{n}\right]\right]\left\{\left\{y_{1}, y_{2}, \ldots, y_{n}\right\}\right\} .
$$


We show that the functor $G$ satisfies the condition of Lemma (2.4). Let $U$ be an arbitrary $k$-vector space which we consider as a variable and

$$
\Phi=\left(\varphi_{1}, \varphi_{2}, \ldots, \varphi_{n}\right) \in \Gamma_{n k}(D(U)) .
$$

So we have

$$
\varphi_{i}=x_{i}+\phi_{\imath} \quad \text { with } \phi_{i}=\phi_{i}(x) \in U\left[\left[x_{1}, x_{2}, \ldots, x_{n}\right]\right]
$$

for $1 \leq i \leq n$. Let

$$
\phi_{i}(x)=\sum_{\left(l_{1}, l_{2}, \ldots, l_{n}\right) \in \mathbb{N}^{n}} a_{i}^{\left(l_{1}, l_{2}, \ldots, l_{n}\right)} x_{1}^{l_{1}} x_{2}^{l_{2}} \cdots x_{n}^{l_{n}} .
$$

with

$$
a_{i}^{\left(l_{1}, l_{2}, \ldots, l_{n}\right)} \in U
$$

Since

$$
U \cdot U=0
$$

in the algebra $D(U)$, the differential equations

$$
F(\Phi)=0
$$

for every $F \in I$ is translated to a system of $k$-linear equations among the coefficients

$$
a_{i}^{\left(l_{1}, l_{2}, \ldots, l_{n}\right)} \in U
$$

of $\phi_{i}$. Namely there exists a system of $k$-linear forms

$$
H_{\alpha}\left(\ldots, a_{i}^{\left(l_{1}, l_{2}, \ldots, l_{n}\right)}, \ldots\right)(\alpha \in J)
$$

in

$$
a_{i}^{\left(l_{1}, l_{2}, \ldots, l_{n}\right)} \in U
$$

indexed by an appropriate set $J$ such that the following conditions are equivalent:

(1) $\Phi \in L_{G}(U)=G(D(U)$;

(2) $F(\Phi)=0$ for every $F \in I$;

(3) $H_{\alpha}\left(\ldots, a_{i}^{\left(l_{1}, l_{2}, \ldots, l_{n}\right)}, \ldots\right)=0$ for every $\alpha \in J$.

Here of course in the linear forms

$$
H_{\alpha}\left(\ldots, a_{i}^{\left(l_{1}, l_{2}, \ldots, l_{n}\right)}, \ldots\right)(\alpha \in J)
$$


only a finite number of the coefficients

$$
a_{i}^{\left(l_{1}, l_{2}, \ldots, l_{n}\right)}
$$

are involved. Furthermore since we choose the vector space $U$ as a variable, the system (2.5.1) is independent of the choice of a vector space $U$. More precisely let $U^{\prime}$ be another $k$-vector space and

$$
\Phi^{\prime}=\left(\varphi_{1}^{\prime}, \varphi_{2}^{\prime}, \ldots, \varphi_{n}^{\prime}\right) \in \Gamma_{n k}\left(D\left(U^{\prime}\right)\right) .
$$

So we have

$$
\varphi_{\imath}^{\prime}=x_{\imath}+\phi_{\imath}^{\prime} \quad \text { with } \phi_{i}^{\prime}=\phi_{i}^{\prime}(x) \in U^{\prime}\left[\left[x_{1}, x_{2}, \ldots, x_{n}\right]\right]
$$

for $1 \leq i \leq n$. Let

$$
\phi_{i}^{\prime}(x)=\sum_{\left(l_{1}, l_{2}, \ldots, l_{n}\right) \in \mathbf{N}^{n}} a_{\imath}^{\left(l_{1}, l_{2}, \ldots, l_{n}\right)} x_{1}^{l_{1}} x_{2}^{l_{2}} \cdots x_{n}^{l_{n}}
$$

with

$$
a_{i}^{\prime\left(l_{1}, l_{2}, \ldots, l_{n}\right)} \in U^{\prime}
$$

Then the following conditions are equivalent:

(1) $\Phi^{\prime} \in L_{G}\left(U^{\prime}\right)=G\left(D\left(U^{\prime}\right)\right.$;

(2) $F\left(\Phi^{\prime}\right)=0$ for every $F \in I$;

(3) $H_{\alpha}\left(\ldots, a_{i}^{\prime\left(l_{1}, l_{2}, \ldots, l_{n}\right)}, \ldots\right)=0$ for every $\alpha \in J$.

Let now $V$ and $W$ be $k$-vector spaces and

$$
\Phi=\left(\varphi_{1}, \varphi_{2}, \ldots, \varphi_{n}\right) \in \Gamma_{n k}(D(V \oplus W))
$$

so that we have

$$
\varphi_{i}=x_{i}+\phi_{i} \quad \text { with } \phi_{i}=\phi_{i}(x) \in(V \oplus W)\left[\left[x_{1}, x_{2}, \ldots, x_{n}\right]\right]
$$

for $1 \leq i \leq n$. Let

$$
\phi_{i}(x)=\sum_{\left(l_{1}, l_{2}, \ldots, l_{n}\right) \in \mathbf{N}^{n}}\left(a_{i}^{\left(l_{1}, l_{2}, \ldots, l_{n}\right)}+b_{i}^{\left(l_{1}, l_{2}, \ldots, l_{n}\right)}\right) x_{1}^{l_{1}} x_{2}^{l_{2}} \cdots x_{n}^{l_{n}}
$$

with

$$
a_{i}^{\left(l_{1}, l_{2}, \ldots, l_{n}\right)} \in V, \quad b_{i}^{\left(l_{1}, l_{2}, \ldots, l_{n}\right)} \in W .
$$

Then it follows from the equivalence of conditions (1), (2), (3) the following conditions are equivalent: 
(1) $\Phi \in L_{G}(V \oplus W)=G(D(V \oplus W))$;

(2) $H_{\alpha}\left(\ldots, a_{i}^{\left(l_{1}, l_{2}, \ldots, l_{n}\right)}, \ldots\right)=0$ and $H_{\alpha}\left(\ldots, b_{i}^{\left(l_{1}, l_{2}, \ldots, l_{n}\right)}, \ldots\right)=0$ for every $\alpha \in J$;

(3) If we set

$$
\begin{aligned}
& \theta_{i}=x_{i}+\sum_{\left(l_{1}, l_{2}, \ldots, l_{n}\right) \in \mathbf{N}^{n}} a_{i}^{\left(l_{1}, l_{2}, \ldots, l_{n}\right)} x_{1}^{l_{1}} x_{2}^{l_{2}} \cdots x_{n}^{l_{n}} \\
& \pi_{i}=x_{i}+\sum_{\left(l_{1}, l_{2}, \ldots, l_{n}\right) \in \mathbf{N}^{n}} b_{i}^{\left(l_{1}, l_{2}, \ldots, l_{n}\right)} x_{1}^{l_{1}} x_{2}^{l_{2}} \cdots x_{n}^{l_{n}}
\end{aligned}
$$

for $1 \leq i \leq n$ and

$$
\Theta=\left(\theta, \theta_{2}, \ldots, \theta_{n}\right), \quad \Pi=\left(\pi_{1}, \pi_{2}, \ldots, \pi_{n}\right),
$$

then $\Theta \in G(D(V))$ and $\Pi \in G(D(W))$.

Hence the functor

$$
L_{G}:(V e c / k) \rightarrow(S e t)
$$

transforms a product to a product so that we can apply Lemma (2.4) to $G$.

Remark (2.5.1). Let $G$ be a Lie-Ritt functor. It follows from the Proof of Corollary (2.5) that the $k$-vector space $\operatorname{Lie}_{k} G$ is isomorphic to the $k$-subspace of $\Theta_{n k}$ defined by the linear equations

$$
H_{\alpha}\left(\ldots, a_{i}^{\prime\left(l_{1}, l_{2}, \ldots, l_{n}\right)}, \ldots\right)=0 .
$$

The $k$-vector space Lie $G$ is isomorphic to the $k$-subspace

$$
\begin{aligned}
& \left\{\phi_{1} \partial / \partial x_{1}+\phi_{2} \partial / \partial x_{2}+\cdots+\partial_{n} \partial / \partial x_{n} \in \Theta_{n k} \mid\right. \\
& \left.\quad \phi_{i}(x)=\sum_{\left(l_{1}, l_{2}, \ldots, l_{n}\right) \in \mathbf{N}^{n}} a_{i}^{\left(l_{1}, l_{2}, \ldots, l_{n}\right)} x_{1}^{l_{1}} x_{2}^{l_{2}} \ldots x_{n}^{l_{n}}, H_{\alpha}\left(\ldots, a_{i}^{\left(l_{1}, l_{2}, \ldots, l_{n}\right)}, \ldots\right)=0\right\}
\end{aligned}
$$

Now we analyze a familiar group functor.

EXAmple (2.6). Let $W$ be a $k$-vector space. For a $k$-algebra $R$, we denote by $\mathrm{GL}_{R}\left(R \otimes_{k} W\right)$ the automorphism group of the $R$-module $R \otimes_{k} W$. So we get a group functor

$$
\mathbf{G L}(W):(A l g / k) \rightarrow(G r p), \quad R \mapsto \mathrm{GL}_{R}\left(R \otimes_{k} W\right) .
$$

Since the functor $\mathbf{G L}(W)$ satisfies the condition of Lemma (2.4), Lie $\mathbf{G L}(W)$ is a $k$-vector space. 
Lemma (2.6.1). We have a canonical isomorphism

$$
\text { Lie } \mathbf{G} \mathbf{L}(W) \simeq \text { End } W
$$

of $k$-vector spaces. Here we denote by End $W$ the $k$-algebra of all endomorphisms of the $k$-vector space $W$.

Proof. Let $k[\varepsilon]$ be the ring of dual numbers and $q: k[\varepsilon] \rightarrow k$ the $k$-algebra homomorphism defined by $q(\varepsilon)=0$. So we have

$$
\begin{aligned}
\text { Lie } \mathbf{G L}(W)=\operatorname{Ker} q_{*} & =\left\{\varphi \in \mathrm{GL}_{k[\varepsilon]}\left(k[\varepsilon] \otimes_{n} W\right) \mid q_{*} \varphi=0\right\} \\
& =\left\{\varphi \in \mathrm{GL}_{k[\varepsilon]}\left(k[\varepsilon] \bigotimes_{n} W\right) \mid \varphi=I+\varepsilon \phi \text { with } \phi \in \text { End } W\right\} .
\end{aligned}
$$

Therefore we get a bijection

$$
\text { Lie } \mathbf{G L}(W) \rightarrow \text { End } W, \quad \varphi \mapsto \phi .
$$

As in the proof of Lemma (2.3), it follows from the definition of the $k$-vector space structure on Lie $\mathbf{G L}(W)$ that this bijection is an isomorphism of the $k$-vector spaces.

LEMMA (2.7). If we have a morphism of group functors

$$
f: G_{1} \rightarrow G_{2}
$$

on the category $(A l g / k)$ of $k$-algebras, then $f$ induces a map

$$
f_{*}: \text { Lie } G_{1} \rightarrow \text { Lie } G_{2} .
$$

If the functors $G_{1}, G_{2}$ satisfies Condition (2.4) so that Lie $G_{1}$, Lie $G_{2}$ are k-vector spaces, then

$$
f_{*}: \text { Lie } G_{1} \rightarrow \text { Lie } G_{2}
$$

is $k$-linear.

Proof. The lemma follows from the definition.

Let $G$ be a group functor on $(A l g / k)$ satisfying Condition (2.4) so that Lie $G$ is a $k$-vector space. Let $k[\varepsilon]$ be the ring of dual numbers. The structure morphism

$$
k \rightarrow k[\varepsilon]
$$

induces a homomorphism 


$$
i: G(k) \rightarrow G(k[\varepsilon])
$$

of groups. So for $g \in G(k)$, we can define

$$
i_{g}: G(k[\varepsilon]) \rightarrow G(k[\varepsilon])
$$

by

$$
h \mapsto i(g)^{-1} h i(g)
$$

Since $i_{g}$ leaves Lie $G=\operatorname{Ker} p_{*}$ invariant, we get a homomorphism

$$
G(k) \rightarrow \mathrm{GL}_{k}(\text { Lie } G)
$$

of groups.

So far we worked over a field $k$. We may however the field $k$ by a ring $R$ and a $k$-vector space by a free $R$-module. So the dimention of a $k$-vector space is replaced by the rank of a free $R$-module. Therefore for example Lie $G$ is defined for a group functor

$$
G:(A \lg / R) \rightarrow(G r p)
$$

as follows. Namely let $V_{1}$ be a free $R$-module of rank 1 . Then

$$
D\left(V_{1}\right)=R \oplus V_{1}
$$

is an $R$-algebra if we define the product of elements of $V_{1}$ to be 0 . Let

$$
p: D\left(V_{1}\right) \rightarrow R
$$

be the projection which is an $R$-algebra homomorphism. We set

$$
\text { Lie } G=\operatorname{Ker} p_{*}
$$

where

$$
p_{*}: G\left(D\left(V_{1}\right)\right) \rightarrow G(R)
$$

is an induced homomorphism of groups by the projection $p$. As in the case of the field $k$, Lie $G$ is defined using the ring $R[\varepsilon]$ of dual numbers over $R$. Let us define a homomorphism of $R$-algebra

$$
q: R[\varepsilon] \rightarrow R
$$

by $q(\varepsilon)=0$ so that we have a homomorphism of groups

$$
q_{*}: G(R[\varepsilon]) \rightarrow G(R)
$$


induced by $q$. Then Lie $G=\operatorname{Ker} q_{*}$. To define an $R$-module structure on Lie $G$, we have to introduce the functor $L_{G}$. For a free $R$-module $V, D(V)=R \oplus V$ is an $R$-algebra if we define the product of any two elements of $V$ to be 0 . We denote by $p$ the projection $D(V) \rightarrow R$ which is an $R$-algebra homomorphism. So $p$ induces a homomorphism of groups

$$
p_{*}: G(D(V)) \rightarrow G(R) .
$$

If we set

$$
L_{G}(V)=\operatorname{Ker} p_{*}
$$

then we get a functor

$$
L_{G}:(\mathrm{Fmod} / \mathrm{R}) \rightarrow(\mathrm{Set}),
$$

where $(F \bmod / R)$ is the category of free $R$-modules. The Proof of Lemma (2.4) allows us to prove that if the functor $L_{G}$ transforms a product to a product, then Lie $G$ is an $R$-module. We can prove the following Lemmas as over the field $k$.

Lemma (2.9). Let $\Theta_{n R}$ be the free $R$-module

$$
\begin{gathered}
R\left[\left[x_{1}, x_{2}, \ldots, x_{n}\right]\right]^{n} \partial / \partial x_{1}+R\left[\left[x_{1}, x_{2}, \ldots, x_{n}\right]\right]^{n} \partial / \partial x_{2} \\
+\cdots+R\left[\left[x_{1}, x_{2}, \ldots, x_{n}\right]\right]^{n} \partial / \partial x_{n} .
\end{gathered}
$$

of all formal vector fields. Then we have a canonical isomorphism

$$
D: \text { Lie } \Gamma_{n R} \rightarrow \Theta_{n R}
$$

of $R$-modules.

Lemma (2.10). For a free $R$-module $W$, we have a canonical isomorphism

$$
\text { Lie } \mathbf{G L}(W) \simeq \operatorname{End}_{R} W
$$

of $R$-modules. Here we denote by $\operatorname{End}_{R} W$ the $R$-algebra of all endomorphisms of the $R$-module $W$.

Lemma (2.11). Let $G$ be a Lie-Ritt functor defined over a field $k$. Then for a $k$-algebra $R$, Lie $G_{R}$ is a free $R$-module. We have a functorial isomorphism

$$
\text { Lie } G_{R}=R \otimes_{k} \text { Lie } G \text {. }
$$

Proof. We use the notation of the Proof of Corollary (2.5) so that $G$ is a 
group subfunctor of $\boldsymbol{\Gamma}_{n k}$ defined by a differential ideal $I$ of

$$
k\left[\left[x_{1}, x_{2}, \ldots, x_{n}\right]\right]\left\{\left\{y_{1}, y_{2}, \ldots, y_{n}\right\}\right\} .
$$

So the argument of the Proof of Corollary (2.5) allows us to prove the following. Let $U$ be a free $R$-module and

$$
\Phi=\left(\varphi_{1}, \varphi_{2}, \ldots, \varphi_{n}\right) \in L_{\Gamma_{n k}}(U) .
$$

So that we have

$$
\varphi_{i}=x_{i}+\phi_{i} \quad \text { with } \phi_{i}=\phi_{i}(x) \in U\left[\left[x_{1}, x_{2}, \ldots, x_{n}\right]\right]
$$

for $1 \leq i \leq n$. Let

$$
\phi_{\imath}(x)=\sum_{\left(l_{1}, l_{2}, \ldots, l_{n}\right) \in \mathbf{N}^{n}} a_{\imath}^{\left(l_{1}, l_{2}, \ldots, l_{n}\right)} x_{1}^{l_{1}} x_{2}^{l_{2}} \cdots x_{n}^{l_{n}}
$$

with

$$
a_{i}^{\left(l_{1}, l_{2}, \cdots, l_{n}\right)} x^{l_{1}} x_{2}^{l_{2}} \cdots x_{n}^{l_{n}} \in U .
$$

So by the argument of the Proof the following conditions are equivalent:

(1) $\Phi \in L_{G}(U)=G(D(U)$;

(2) $F(\Phi)=0$ for every $F \in I$;

(3) $H_{\alpha}\left(\ldots, a_{\imath}^{\left(l_{1}, l_{2}, \ldots, l_{n}\right)}, \ldots\right)=0$ for every $\alpha \in J$

So as in the Proof of Corollary (2.5), the functor

$$
L_{G_{R}}:(F \bmod / R) \rightarrow(S e t)
$$

transforms a product to a product. Hence the Proof of Lemma (2.4) shows that Lie $G_{R}$ is an $R$-submodule of $\Theta_{n R}$ defined by $H_{\alpha}=0$. Since the form $H_{\alpha}$ is $k$-linear, we have an isomorphism

$$
\text { Lie } G_{R} \simeq R \otimes_{k} \text { Lie } G
$$

as $R$-modules (cf. Remark (2.5.1)).

The above argument allows us to prove the following result (cf. [S.G.A.D], Exposé II , Proposition 3.4).

Lemma (2.12). Let

$$
G:(A l g / k) \rightarrow(G r p)
$$


be a group functor. If the functor

$$
L_{G_{R}}:(F \bmod / R) \rightarrow(S e t)
$$

transforms a product to a product for every $k$-algebra $R$, then Lie $G_{R}$ is an $R$-module and we have an isomorphism

$$
\text { Lie } G_{R} \simeq R \otimes_{k} \text { Lie } G
$$

of $R$-modules.

Let now $G$ be a Lie-Ritt functor defined over a filed $k$. It follows from (2.8) interpreted over $k$-algebras and from Lemma (2.11) that we get a morphism of group functors

$$
G \rightarrow \mathbf{G L}(\text { Lie } G)
$$

So we get by (2.7)

$$
f: \text { Lie } G \rightarrow \mathbf{G L}(G)=\operatorname{End}(\text { Lie } G) .
$$

The morphism defines a product on Lie $G$. We denote by $f_{x}$ the image of $x \in$ Lie $G$ by the map $f$ in (2.13) so that

$$
f_{x} \in \operatorname{End}(\text { Lie } G) .
$$

Hence we get a $k$-linear map

$$
f_{x}: \text { Lie } G \rightarrow \text { Lie } G \text {. }
$$

We set

$$
[x, y]=f_{x}(y) \text { for } x, y \in \text { Lie } G \text {. }
$$

Proposition (2.14). Let

$$
\Phi, \Psi \in \operatorname{Lie} \boldsymbol{\Gamma}_{n k}
$$

and

$$
D: \text { Lie } \Gamma_{n k} \rightarrow \Theta_{n k}
$$

be the isomorphism of Lemma (2.3). Then we have

$$
D([\Phi, \Psi])=[D(\Phi), D(\Psi)]
$$

the latter being equal to 


$$
D(\Phi) D(\Psi)-D(\Psi) D(\Phi)
$$

by definition. Namely the $k$-vector space Lie $\boldsymbol{\Gamma}_{n k}$ forms a Lie algebra with respect to the product [,] which is isomorphic to the Lie algebra $\Theta_{n k}$ of all formal vector fields.

Proof. We may assume for the simplicity $n=1$. Let $k[\varepsilon]$ be the ring of all dual numbers. We prepare a copy $k\left[\varepsilon^{\prime}\right]$ of $k[\varepsilon]$. So let

$$
\Phi=x+\varepsilon \varphi(x), \Psi=x+\varepsilon^{\prime} \psi(x) \quad \text { with } \varphi(x), \phi(x) \in k[[x]] .
$$

Hence we have

$$
D(\Phi)=\varphi(x) d / d x, \quad D(\Psi)=\phi(x) d / d x .
$$

Let us calculate in $k\left[\varepsilon, \varepsilon^{\prime}\right][[x]]$

$$
U=(x+\varepsilon \varphi(x))^{-1} \circ\left(x+\varepsilon^{\prime} \phi(x)\right) \circ(x+\varepsilon \varphi(x))-\left(x+\varepsilon^{\prime} \phi(x)\right) .
$$

We need

Lemma (2.15). Let $f(x), g(x) \in R[[x]]$ for a ring $R$. Then we have in

$$
f(x+\varepsilon g(x))=f(x)+\varepsilon g(x) f^{\prime}(x) \text { in } R[\varepsilon][[x]]\left(\varepsilon^{2}=0\right) .
$$

Proof of lemma. In fact since $\varepsilon^{2}=0$, if $f(x)=x^{n}$, then we have

$$
(x+\varepsilon g(x))^{n}=x^{n}+\varepsilon g(x) n x^{n-1}
$$

by the binomial expansion theorem. The lemma follows from this observation.

Repeated applications of Lemma (2.15) give us

$$
\begin{aligned}
& U=(x-\varepsilon \varphi(x)) \circ\left(x+\varepsilon^{\prime} \phi(x)\right) \circ(x+\varepsilon \varphi(x))-\left(x+\varepsilon^{\prime} \phi(x)\right) \\
& =(x-\varepsilon \varphi(x)) \circ\left(x+\varepsilon \varphi(x)+\varepsilon^{\prime} \phi(x+\varepsilon \varphi(x))-\left(x+\varepsilon^{\prime} \phi(x)\right)\right. \\
& =(x-\varepsilon \varphi(x)) \circ\left(x+\varepsilon \varphi(x)+\varepsilon^{\prime}\left(\phi(x)+\varepsilon \phi^{\prime}(x) \varphi(x)\right)-\left(x+\varepsilon^{\prime} \phi(x)\right)\right. \\
& =x+\varepsilon \varphi(x)+\varepsilon^{\prime}\left(\phi(x)+\varepsilon \psi^{\prime}(x) \varphi(x)\right)-\varepsilon \varphi\left(x+\varepsilon \varphi(x)+\varepsilon^{\prime}(\phi(x)\right. \\
& \left.\left.+\varepsilon \phi^{\prime}(x) \varphi(x)\right)\right)-\left(x+\varepsilon^{\prime} \phi(x)\right) \\
& =x+\varepsilon \varphi(x)+\varepsilon^{\prime}\left(\phi(x)+\varepsilon \psi^{\prime}(x) \varphi(x)\right)-\varepsilon \varphi\left(x+\varepsilon^{\prime} \phi(x)+\varepsilon(\varphi(x)\right. \\
& \left.+\varepsilon^{\prime} \phi^{\prime}(x) \varphi(x)\right)-\left(x+\varepsilon^{\prime} \phi(x)\right) \\
& =x+\varepsilon \varphi(x)+\varepsilon^{\prime}\left(\phi(x)+\varepsilon \psi^{\prime}(x) \varphi(x)\right)-\varepsilon \varphi\left(x+\varepsilon^{\prime} \phi(x)\right)-\left(x+\varepsilon^{\prime} \phi(x)\right) \\
& =x+\varepsilon \varphi(x)+\varepsilon^{\prime} \phi(x)+\varepsilon \varepsilon^{\prime} \phi^{\prime}(x) \varphi(x)-\varepsilon \varphi(x)-\varepsilon \varepsilon^{\prime} \phi(x) \varphi^{\prime}(x) \\
& -\left(x+\varepsilon^{\prime} \phi(x)\right) \\
& =\varepsilon \varepsilon^{\prime}\left(\phi^{\prime}(x) \varphi(x)-\phi(x) \varphi^{\prime}(x)\right) \text {. }
\end{aligned}
$$


Let $F$ be a formal group over a ring $R$. Then

$$
F(x, y)=x+y+B(x, y)+\text { terms of degree } \geq 3
$$

(cf. [S] LG, Chap. 4, §7). Then usually the Lie $F$ of the formal group $F$ is defined as the free $R$-module $R^{n}$ equipped with the product

$$
[x, y]_{F}=B(x, y)-B(y, x)
$$

for $x, y \in R^{n}$ (cf. [S] LG, Chap. 5, §1). If

$$
f: F \rightarrow G
$$

is a morphism of formal groups over $R$, then

$$
f_{1}: \text { Lie } F \rightarrow \text { Lie } G
$$

induces a homomorphism of $R$-Lie algebras. Here $f_{1}$ is the linear part of $f$ (cf. [S] loc. cit.). We can also consider the Lie algebra of the associated Lie-Ritt functor $\mathbf{F}$.

Proposition (2.16). Let $F$ be formal group over a commutative ring $R$. Then we have a canonical isomorphism

\section{Lie $F \simeq$ Lie $\mathbf{F}$}

of $R$-Lie algebras.

Proof. This follows from the Proof of Proposition (2.14) and from the formula

$$
x y x^{-1}=y+[x, y]_{F}+\text { terms of degree } \geq 3
$$

where we denote $F(x, y), \Theta(x)$ respectively by $x y, x^{-1}$ (cf. [S] LG, Chap. 4, §7, Formula 3).

\section{§3. Construction of $\mathscr{K}$ and $\mathscr{L}$}

Let $L / K$ be an ordinary differential field extension with derivation $\delta$. We assume that the field extension $L / K$ satisfies the following condition.

(F.C) The field $L$ is finitely generated over $K$ as an abstract field.

We have the universal Taylor morphism 


$$
i: L \rightarrow L^{\natural}[[t]]: i(a)=\sum_{n=0}^{\infty}\left(\delta^{n} a / n !\right) t^{n} \quad \text { for } a \in L
$$

(cf. [U3], §1). Here as in [U3], $L^{\natural}$ denotes the abstract field structure of the differential field $L$ and $t$ is an independent variable over $L$. We add $\sharp$ when we want to emphasize that we consider the abstract field structure of a differential ring.

So the power series ring $L^{\natural}[[t]]$ is a differential ring with derivation $d / d t$ and the universal Taylor morphism $i$ is a morphism of differential rings, i.e. we have

$$
i(\delta a)=\frac{d}{d t} i(a) \quad \text { for every } a \in L
$$

Let $\left\{u_{1}, u_{2}, \ldots, u_{n}\right\}\left(u_{i} \in L\right.$ for $\left.1 \leq i \leq n\right)$ be a transcendence basis of $L$ over $K$ so that $K\left[u_{1}, u_{2}, \ldots, u_{n}\right]$ is isomorphic to a polynomial ring (here rigorously speaking, we have to write $L^{\natural}, K^{\natural}$ for $L$ and $K$, which would make the notation complicated). Hence we have the $K$-derivations

$$
\partial / \partial u_{i}: K\left(u_{1}, u_{2}, \ldots, u_{n}\right) \rightarrow K\left(u_{1}, u_{2}, \ldots, u_{n}\right) \text { for } 1 \leq i \leq n .
$$

Since $L$ is algebraic over $K\left(u_{1}, u_{2}, \ldots, u_{n}\right)$, each $K$-derivation

$$
\partial / \partial u_{i}: K\left(u_{1}, u_{2}, \ldots, u_{n}\right) \rightarrow K\left(u_{1}, u_{2}, \ldots, u_{n}\right) \text { for } 1 \leq i \leq n
$$

can be extended to the unique derivation $L^{\natural} \rightarrow L^{\natural}$ which we denote by the same symbol $\partial / \partial u_{\imath}$. The field $L^{\natural}$ endowed with the derivations $\partial / \partial u_{\imath}$ will be denoted by $L^{\#}$. Now we have in $L^{\natural}[[t]]\left[t^{-1}\right]$ two kinds of derivations: (i) the differentiation $d / d t$; (ii) the derivations $\partial / \partial u_{i}$ applied on the coefficients of Laurent series. Namely we set

$$
\frac{\partial}{\partial u_{i}}\left(\sum_{-\infty \ll n}^{\infty} a_{n} t^{n}\right)=\sum_{-\infty \ll n}^{\infty} \frac{\partial a_{n}}{\partial u_{\imath}} t^{n} \quad \text { for } \sum_{-\infty \ll n}^{\infty} a_{n} t^{n} \in L^{n}[[t]]\left[t^{-1}\right] .
$$

So we get a differential field

$$
\left(L^{\natural}[[t]]\left[t^{-1}\right],\left\{d / d t, \partial / \partial u_{1}, \partial / \partial u_{2}, \ldots, \partial / \partial u_{n}\right\}\right) .
$$

Its constant field is the algebraic closure of $K^{\natural}$ in $L^{\natural}$ :

$$
\begin{gathered}
K^{\natural}\left(\subset L^{\natural} \subset L^{\natural}[[t]]\left[t^{-1}\right]\right), \\
K^{\natural}=\left\{\sum_{-\infty \ll n}^{\infty} a_{n} t^{n} \in L^{\natural}[[t]]\left[t^{-1}\right] \mid a_{0} \in K^{\natural} \text { and } a_{i}=0 \text { for } i \neq 0\right\} .
\end{gathered}
$$

The differential field 


$$
\left(L^{\natural}[[t]]\left[t^{-1}\right],\left\{d / d t, \partial / \partial u_{1}, \partial / \partial u_{2}, \ldots, \partial / \partial u_{n}\right\}\right)
$$

will be denoted by $L^{\#}[[t]]\left[t^{-1}\right]$.

Let $\left\{v_{1}, v_{2}, \ldots, v_{n}\right\}\left(v_{i} \in L^{\natural}\right.$ for $\left.1 \leq i \leq n\right)$ be another transcendence basis of the field extension $L^{\natural} / K^{\natural}$. So we can introduce the derivation $\partial / \partial v_{\imath}: L^{\natural} \rightarrow$ $L^{\natural}$ for $1 \leq i \leq n$ as above.

Lemma (3.1). The jacobians $\left|\partial v_{\imath} / \partial u_{j}\right|,\left|\partial u_{i} / \partial v_{j}\right| \in L^{\natural}$ are not equal to 0 .

Proof. We know that the dimension of the $L^{\natural}$-vector space $\operatorname{Der}_{K^{4}}\left(L^{\natural}\right)$ is equal to the transcendence degree tr. $\mathrm{d}[L: K]=n$ and that

$$
\left\{\partial / \partial u_{1}, \partial / \partial u_{2}, \ldots, \partial / \partial u_{n}\right\}
$$

and

$$
\left\{\partial / \partial v_{1}, \partial / \partial v_{2}, \ldots, \partial / \partial v_{n}\right\}
$$

form respectively basis of the $L^{\natural}$-vector space $\operatorname{Der}_{K^{4}}\left(L^{\natural}\right)$. So we can find $a_{i j} \in$ $L$ such that

$$
\frac{\partial}{\partial u_{i}}=\sum_{j=1}^{n} a_{i j} \frac{\partial}{\partial u_{j}}
$$

for $1 \leq i \leq n$ with $\left|a_{i j}\right| \neq 0$. It follows from (3.2)

$$
\frac{\partial v_{j}}{\partial u_{i}}=a_{i j}
$$

so that $\left|\partial v_{j} / \partial u_{i}\right| \neq 0$. For the same reason we conclude $\left|\partial u_{j} / \partial v_{i}\right| \neq 0$.

Let us denote by

$$
\operatorname{Hom}_{K \text {-vect }}(L, L)
$$

the $L$-algebra of all $K$-linear endomorphisms of the $K$-vector space $L$. Lemma (3.1) shows that if we consider an $L^{\natural}$-subalgebra of

$$
\operatorname{Hom}_{K \text {-vect }}(L, L)
$$

generated by the $\partial / \partial u_{i}$, then the $L^{\natural}$-subalgebra is uniquely determined. So the definition of any differential $L^{\#}$-subalgebra of $L^{\#}[[t]]\left[t^{-1}\right]$ is independent of the choice of a transcendence basis of $L / K$. Since there is no reason to distinguish $d / d t$ from the other partial derivations, we may denote $d / d t$ by $\partial / \partial t$. 
Definition 3.3. We denote by $\mathscr{K}_{L / K}$ a subfield of $L^{\#}[[t]]\left[t^{-1}\right]$ generated by $L^{\#}$ and $i(K)$, which is a differential subfield of

$$
\left(L^{\#}[[t]]\left[t^{-1}\right],\left\{\partial / \partial u_{1}, \partial / \partial u_{2}, \ldots, \partial / \partial u_{n}, \partial / t\right\}\right) .
$$

$\mathscr{L}_{L / K}$ is a differential subfield of $L^{\#}[[t]]\left[t^{-1}\right]$ generated by $i(L)$ and $\mathscr{K}_{L / K}$. We de note $\mathscr{K}_{L / K}, \mathscr{L}_{L / K}$ respectively by $\mathscr{K}, \mathscr{L}$ when there is no danger of misunderstand ing the starting differential field extension $L / K$.

Example (3.4). Let us analyse Example (2.1) of [U2]. In this example $K=$ $\mathbf{C}(x)$ and $L=K(y)$ with $y=\exp x$, the derivation $\delta$ of $L$ being $d / d x$. So we have $y^{(n)}=y$ for any integer $n \in \mathbf{N}$. Thus since we have $i(x)=x+t$, we have

$$
\mathscr{K}=i(K), L^{\#}=i\left(\mathbf{C}(i(x)) L^{\#}=L^{\#}(t) \subset L^{\#}[[t]]\left[t^{-1}\right] .\right.
$$

It follows from the equality

$$
i(y)=y \exp t
$$

that we have in $L^{\natural}[[t]]\left[t^{-1}\right]$

$$
i(L) L^{\natural}=i(K(y)) L^{\natural}=i(K)(y \exp t) L^{\natural}=i(K) \cdot L^{\natural}(\exp t)=\mathscr{K}(\exp t) .
$$

Hence this subfield is closed under the derivations $\partial / \partial y$ and $\partial / \partial t$ and therefore $\mathscr{L}=\mathscr{K}(\exp t)$. See Examples (3.8), (3.9) and Proposition (3.13).

Now we have the universal Taylor morphism

$$
i_{L}: L^{\#} \rightarrow L^{\natural}\left[\left[w_{1}, w_{2}, \ldots, w_{n}\right]\right],
$$

where $w_{1}, w_{2}, \ldots, \omega_{n}$ are independent variables. So we get a morphism

$$
L^{\#}[[t]]\left[t^{-1}\right] \rightarrow L^{\natural}\left[\left[w_{1}, w_{2}, \ldots, w_{n}, t\right]\right]\left[t^{-1}\right]
$$

of the differential algebras with the $(n+1)$ derivations sending

$$
\sum_{-\infty \ll n}^{\infty} a_{n} t^{n} \text { to } \sum_{-\infty \ll n}^{\infty} i_{L}\left(a_{n}\right) t^{n}
$$

Restricting this morphism to the subfield $\mathscr{L}$, we get a morphism

$$
\left.\iota: \mathscr{L} \rightarrow L^{\natural}\left[w_{1}, w_{2}, \ldots, w_{n}, t\right]\right]\left[t^{-1}\right] .
$$

Let us see what happens in our Example (3.4). We take $u_{1}=y$. Let us calculate the image of $i(y)$ under the morphism $c$. Since 


$$
c(i(y)) \in L^{\natural}\left[\left[w_{1}, t\right]\right]\left[t^{-1}\right]
$$

let us set $Y\left(w_{1}, t\right)=\iota(i(y))$. It follows now from (3.4.1)

$$
Y\left(w_{1}, t\right)=\left(y+w_{1}\right) \exp t \in L^{\natural}[[w, t]]\left[t^{-1}\right] .
$$

This shows that $Y\left(w_{1}, t\right)$ is nothing but the general solution of the differential equation $d y / d x=y$, if we notice that the differential equation $y^{\prime}=y$ is translated into $\frac{\partial Y}{\partial t}=Y$ in the differential algebra $L^{\natural}\left[\left[w_{1}, t\right]\right]\left[t^{-1}\right]$.

Let us study a more general situation. Let $K$ be an ordinary differential field and $L=K\langle y\rangle$ such that

$$
y^{(n)}=F\left(y, y^{\prime}, \ldots, y^{(n-1)}\right) \quad \text { with } F\left(y, y^{\prime}, \ldots, y^{(n-1)}\right) \in K\left[y, y^{\prime}, \ldots, y^{(n-1)}\right]
$$

More precisely we consider the differential equation (3.5) and we define $L$ as a quotient field of

$$
K\{y\} /\left(\text { differential ideal generated by } y^{(n)}-F\left(y, y^{\prime}, \ldots, y^{(n-1)}\right)\right) .
$$

We take

$$
y=u_{1}, y^{(1)}=u_{2}, \ldots, y^{(n-1)}=u_{n}
$$

If we set

$$
c(i(y))=Y(w, t)=Y\left(w_{1}, w_{2}, \ldots, w_{n}, t\right) \in L^{\natural}\left[\left[w_{1}, w_{2}, \ldots, w_{n}, t\right]\right]\left[t^{-1}\right],
$$

then

$$
\frac{\partial^{n} Y}{\partial t^{n}}=F\left(Y, \frac{\partial Y}{\partial t}, \ldots, \frac{\partial^{n-1} Y}{\partial t^{n-1}}\right)
$$

and since $i\left(y^{(i)}\right)(0)=y^{(i)}$,

$$
\frac{\partial^{i} Y(u, 0)}{\partial t^{i}}=y^{(\imath)}+w_{\imath} \in L^{\natural}\left[\left[w_{1}, w_{2}, \ldots, w_{n}\right]\right] .
$$

Namely $Y(u, t)$ is the generic solution of the differential equation (3.5) or is the solution depending on the $n$ parameters $w_{1}, w_{2}, \ldots, w_{n}$. The derivation $\partial / \partial w_{i}$ is really a differentiation of the solution $Y(w, t)$ with respect to the initial value $w_{i}$.

Remark (3.7). In general the field $\mathscr{L}^{\natural}$ is not of finite type over the field $\mathscr{K}^{\natural}$ We can construct such an example by considering a simple differential equation $y^{\prime}=F(y)$ 
Example (3.8). The Weierstraß $\gamma$-function. Let $\mathscr{K}$ be a differential field and $y$ an element of a differential overfield of $\mathscr{K}$ satisfying a differential equation

$$
y^{\prime 2}=4 y^{3}-g_{2} y^{2}-g_{3}
$$

with $g_{2}, g_{3} \in C_{K}$ such that

$$
g_{2}^{3}-27 g_{3}^{2} \neq 0
$$

The equation

$$
Y^{3} Z=4 X^{3}-g_{2} X Z^{2}-g_{3} Z^{3}
$$

defines an elliptic curve $E$ in $\mathbf{P}_{C_{K}}^{3}$ and $\left(1, y, y^{\prime}\right)$ gives us an $L$-valued point

$$
g: \operatorname{Spec} L \rightarrow E
$$

if we set $L=K\left(y, y^{\prime}\right)$. We notice that the differential equation (3.8.1) contains as

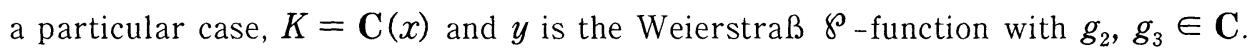
We denote $Y / X, Z / X$ by $u, v$ so that we have $v^{2}=4 u^{3}-g_{2} u^{2}-g_{3}$ and the function field of the elliptic curve $E$ is

$$
C_{K}(u, v) .
$$

Let us take

$$
(X, Y, Z)=(0,0,1)
$$

as the origin of $E$. The $L$-valued point $g$ of $E$ defines a tangent vector $\delta g$ at $g$ and it follows from the definition that

$$
(\delta g)(u)=\delta\left(g^{*}(u)\right)=\delta y=y^{\prime},(\delta g)(v)=\delta\left(g^{*}(v)\right)=\delta\left(y^{\prime}\right)=y^{\prime \prime}
$$

(cf. Example (2.15), [U2]). So we have

$$
l \delta g=v \partial /\left.\partial u\right|_{(u, v)=g} .
$$

If we take $u=u_{1}=y$, then

$$
i(y)=Y(t) \in L^{\natural}[[t]] .
$$

The power series $Y(t)$ satisfies

$$
\left(\frac{\partial Y}{\partial t}\right)^{2}=4 Y^{3}-g_{2} Y-g_{3}
$$

where we identify $C_{K}$ with its image 


$$
i\left(C_{K}\right) \subset L^{\natural} \subset L^{\natural}[[t]]\left[t^{-1}\right] .
$$

The points

$$
(u, v)=\left(y, y^{\prime}\right),(Y, \partial Y / \partial t)
$$

are $L^{\natural}[[t]]$-valued point of $E$. We writing the addition on the elliptic curve $E$ by + , the sum

$$
(\tilde{X}, \tilde{Y}, 1)=(Y, \partial Y / \partial t)-\left(y, y^{\prime}\right)
$$

of the two points on $E$ is an $L^{\natural}[[t]]$-valued point of the elliptic curve $E$. Since the vector field $v \partial / \partial u$ on $E$ is translation invariant, it follows from (3.8.2) and from Lemma 16 of [U1] that the $L^{\natural}[[t]]\left[t^{-1}\right]$-valued point $(\tilde{X}, \tilde{Y}, 1)$ of $E$ satisfies (3.8.2) too. The equation (3.8.2) is written in terms of $(\tilde{X}, \tilde{Y})$ as a system

$$
\left\{\begin{array}{l}
\frac{\partial \tilde{X}}{\partial t}=-6 \tilde{Y}^{2}+g_{2} \tilde{X} \tilde{Y} \\
\frac{\partial \tilde{Y}}{\partial t}=-\frac{g_{2}}{4} \tilde{X} \tilde{Y}-g_{3} \tilde{X}^{2}+g_{2} \tilde{Y}^{2}-\frac{1}{2} .
\end{array}\right.
$$

Since the initial condition

$$
(\tilde{X}(0), \tilde{Y}(0), 1)=\left(y, y^{\prime}\right)-\left(y, y^{\prime}\right)
$$

at $t=0$ is the origin $(0,0,1)$, it follows from the equation (3.8.3) that all the coefficients of the power series $\tilde{X}, \tilde{Y}$ are in $K^{\natural}$. So

$$
\mathscr{K}(Y, \partial Y / \partial t)=\mathscr{K}\left((Y, \partial Y / \partial t)-\left(y, y^{\prime}\right)\right)=\mathscr{K}(\tilde{X}, \tilde{Y}) .
$$

Since the coefficients of $\tilde{X}, \tilde{Y}$ are in $\mathscr{K}$,

$$
\partial \tilde{X} / \partial y=\partial \tilde{Y} / \partial y=0
$$

and hence $\mathscr{K}(\tilde{X}, \tilde{Y})$ is closed under the derivation $\partial / \partial y$. Thus

$$
\mathscr{K}(Y, \partial Y / \partial t)=\mathscr{K}(\tilde{X}, \tilde{Y})=\mathscr{L} .
$$

See Examples (3.4), (3.9) and Proposition (3.13).

Example (3.9). Let $M / K$ be an ordinary differential extension and

$$
A=\left(a_{t, 1}\right) \in M_{m}(K)
$$

a square matrix of degree $m$. If a matrix 


$$
Y=\left(y_{i j}\right) \in M_{m}(M)
$$

satisfies a linear differential equation

$$
Y^{\prime}=A Y,
$$

where we denote by $Y^{\prime}$ a matrix $\left(y_{i, j}^{\prime}\right)_{1 \leq \imath, j \leq m}$. Then a subfield $L=K\left(y_{i j}\right)_{1 \leq i, j \leq m}$ of the differential field $M$ is colsed under the derivation. If we denote the matrix

$$
\left(i\left(y_{i j}\right)\right) \in M_{m}\left(L^{4}[[t]]\left[t^{-1}\right]\right)
$$

by $i(Y)$. Then if we set

$$
\tilde{Y}(t)=i(Y) Y^{-1},
$$

then the matrix $\tilde{Y}(t)$ satisfies a differential equation

$$
\frac{d \tilde{Y}}{d t}=i(A) \tilde{Y}
$$

where $i(A)=\left(i\left(a_{i j}\right)\right) \in M_{m}\left(L^{\natural}[[t]]\left[t^{-1}\right]\right)$. Equation (3.11) is an identity between the matrices in $M_{m}\left(L^{\natural}[[t]]\left[t^{-1}\right]\right)$. Since $i(A) \in M_{m}\left(K^{\natural}[[t]]\right)$ and $\tilde{Y}(0)=$ $I_{m}$, we can show by induction on $l$, using (3.11) that the coefficients of $\tilde{Y}$ in $t^{l}$ is in $K^{\natural}$ for $l=0,1,2, \ldots$ Namely

$$
\tilde{Y}(t) \in M_{n}\left(K^{\natural}[[t]]\right) .
$$

Now in the field $L^{\natural}[[t]]\left[t^{-1}\right]$, we have

$$
\begin{gathered}
L^{\natural} . i(L)=L^{\natural} i(K(Y))=L^{\natural} . i(K)(i(Y)) \\
\quad=L^{\natural} . i(K)\left(i(Y) Y^{-1 \natural}\right)=\mathscr{K}(\tilde{Y}(t)) .
\end{gathered}
$$

Here we denote for example by $L^{\natural} . i(L)$ a subfield of $L^{\natural}[[t]]\left[t^{-1}\right]$ generated by $L$ and $i(L)$. So if we take a transcendence basis $u_{1}, u_{2}, \ldots, u_{n}$ of $L / K$ and introduce the derivations $\partial / \partial u_{j}$ to obtain $L^{\#}$, then by $(3.12) L^{\#}, i(Y)=\mathscr{K}(\tilde{Y}(t))$ is closed under the derivations $\partial / \partial u_{j}, \partial / \partial t$ and hence coincides with $\mathscr{L}$ (cf. Examples (3.4), (3.8) and Proposition (3.13)).

In Examples (3.4), (3.8) and (3.9), the extensions $L / K$ are $G$-primitive and we have $\mathscr{L}=i(L) . L^{\#}$. In general if we denote by $K_{1}$ the algebraic closure of $K$ in $L$, then the field $C_{\mathscr{K}}$ of all constants of $\mathscr{K}$ is $K_{1}^{\natural}$. We have the following general result.

Proposition (3.13). If $L / K$ is a $G$-primitive extension, then $\mathscr{L}=i(L) \cdot L^{\#}$ and $\mathscr{L} / \mathscr{K}$ is a $G_{C_{\mathscr{K}}}$-primitive extension. 
Proof. It follows from the definition of a $G$-primitive extension that $G$ is an algebraic group scheme defined over the field $C_{K}$ of all constants and that there exists an $L$-valued point $g: \operatorname{Spec} L \rightarrow G$ of the algebraic group $G$ satisfying the following two conditions: (1) There exists a right invariant vector field $\alpha \in$ Lie $G_{K}$ such that

$$
l \delta g=\alpha
$$

(2) We have $K(g)=L$. (See [U1], p. 784 for the definition of the logarithmic differential $l \delta g$.) Let $\operatorname{Spec} A \subset G$ be an affine neighbourhood of the image of the $L$-valued point $g$ and

$$
A=C_{K}\left[z_{1}, z_{2}, \ldots, z_{m}\right]
$$

So we have a $C_{K}$-morphism

$$
g^{*}: A \rightarrow L, \quad\left(z, \mapsto g_{j} \quad \text { for } 1 \leq j \leq m\right)
$$

of $C_{K}$-algebras. We have by definition $\delta g_{j}=(l \delta g) z_{j}$, which is equal to $\alpha(g) z_{j}=$ $\left(a z_{j}\right)(g)$ by (3.13.1) for $1 \leq j \leq m$. Since $\alpha z_{j} \in A \otimes_{C_{K}} K$,

$$
\alpha z_{j}(g)=f_{j}\left(z_{1}, z_{2}, \ldots, z_{m}\right),
$$

where $f_{j}$ is a polynomial with coefficients in $K$. So we have

$$
\delta g_{j}=f_{j}\left(g_{1}, g_{2}, \ldots, g_{m}\right) \text { for } 1 \leq j \leq m .
$$

If we pass from $L$ to $L^{\natural}[[t]]$ through the universal Taylor morphism $i: L \rightarrow$ $L^{\natural}[[t]]$,

$$
\left(i\left(g_{1}\right), i\left(g_{2}\right), \ldots, i\left(g_{m}\right)\right)
$$

gives us an $i(L)$-valued (hence an $L[[t]]$-valued) point of $\operatorname{Spec} A \subset G$ which satisfies

$$
\frac{\partial\left(i\left(g_{j}\right)\right)}{\partial t}=F_{j}\left(i\left(g_{1}\right), i\left(g_{2}\right), \ldots, i\left(g_{m}\right)\right) \text { for } 1 \leq j \leq m
$$

where the polynomials

$$
F_{j} \in K^{\natural}[[t]]\left[Z_{1}, Z_{2}, \ldots, Z_{m}\right] \quad(1 \leq j \leq m)
$$

are the image of $f_{j} \in K\left[Z_{1}, Z_{2}, \ldots, Z_{m}\right]$ by the universal Taylor morphism $K \rightarrow$ $K^{\natural}[[t]]$. We denote this $L[[t]]$-valued point of $G$ by $i(g)$. On the other hand

$$
\left(z_{1}^{\natural}, z_{2}^{\natural}, \ldots, z_{m}^{\natural}\right)
$$


gives us an $L^{\natural}$-valued (hence an $L^{\natural}[[t]]$-valued) point of Spec $A \subset G$, which we denote by $g^{\natural}$. So $i(g) g^{\natural-1}$ is an $L^{\natural}[[t]]$-valued point of $G$. It follows from the construction that $i(g) g^{\natural-1}$ is in fact an $i(L) . L^{\natural}$-valued point. Since

$$
(l d / d t)\left(i(g) g^{\natural-1}\right)=\alpha \in \operatorname{Lie} G_{K} \subset \text { Lie } G_{\mathscr{K}}
$$

by Lemma 16 of [U1], the point $i(g) g^{\natural-1}$ satisfies the differential equation (3.13.3) too. Namely

$$
i(g) g^{\natural-1}=\left(\tilde{g}_{1}, \widetilde{g}_{2}, \ldots, \tilde{g}_{m}\right)
$$

is a power series solution of (3.13.3) and its initial condition at $t=0$ is $1 \in G$. Hence the initial conditions

$$
\widetilde{g}_{j}(0) \in C_{K} \subset K^{\natural} \quad \text { for } 1 \leq j \leq m .
$$

Since the coefficients of the $F_{j}$ are power series with coefficients in $K^{\natural}$, the $\tilde{g}_{j}(t)$ are in $K^{\natural}[[t]]$. So for any $D \in \operatorname{Der}_{K}, L^{\natural}$ we have $D \tilde{g}_{j}(t)=0$ so that

$$
l D\left(i(g) g^{\natural-1}\right)=0
$$

hence in particular

$$
l D\left(i(g) g^{\natural-1}\right) \in \operatorname{Lie} G_{\mathscr{K}} .
$$

By (3.13.5) the subalgebra

$$
i(L) \cdot L^{\natural}=i(K)\left(i(g) \cdot L^{\natural}\right)=i(K)\left(i(g) g^{\natural-1} \cdot L^{\natural}\right)=\mathscr{K}\left(i(g) g^{\natural-1}\right)
$$

of $L[[t]]\left[t^{-1}\right]$ is invariant under the derivations of $L^{\natural} / K^{\natural}$. Therefore $\mathscr{L}=$ $\mathscr{K}\left(i(g) \cdot L^{\#}\right)=i(L) \cdot L^{\#}$. Now the proposition follows from (3.13.4) and (3.13.6).

A $G$-primitive extension is a particular case of a strongly normal extension (cf. Kolchin [K], Chap. VI).

THEOREM (3.14). If $L / K$ is a strongly normal extension with Galois group $G$, then $\mathscr{L} / \mathscr{K}$ is a strongly normal extension with Galois group $G_{K_{1}}$, Here $K_{1}$ is the algebraic closure of the field $K$ in $L$.

Proof. It follows from Theorems (2.13), (3.10) of [U3] that $G$ is an algebraic group scheme over the field $C=C_{K}=C_{L}$ of all constants and there exists a $K$-scheme $X$ with derivation that is a model of a differential field extension $L / K$ such that the algebraic groups $G_{K}$ operates on $X$ and such that we have a $K$-isomorphism 


$$
G_{K} \times_{K} X=G \times{ }_{C} X \rightarrow X \times{ }_{K} X, \quad(g, x) \mapsto(g x, x)
$$

of schemes with derivation. We show $\mathscr{L}=i(L) \cdot L^{*}$ in $L^{\#}[[t]]\left[t^{-1}\right]$. Let $Y=\operatorname{Spec}$ $A$ be a $K$-affine open set of $X$ with $A=K\left[y_{1}, y_{2}, \ldots, y_{m}\right]$. So the subring $A$ of the differential field $L$ is closed under the derivation and $L=K\left(y_{1}, y_{2}, \ldots, y_{m}\right) . K_{1}$ being the algebraic closure of $K$ in $L$, by the Hilbert Nullstellensatz we can find a finite algebraic extension $K_{2}$ of $K_{1}$ such that the $K$-scheme $Y$ has a $K_{2}$-valued point. We consider $L^{\prime}=L \bigotimes_{K_{1}} K_{2}$, which is a finite algebraic extension of $L^{\prime}$ and is a differential field. In the Laurent series ring $L^{\prime \prime}[[t]]\left[t^{-1}\right]$, which is a differential overring of $L^{\natural}[[t]]\left[t^{-1}\right]$, we have by Lemma (1.1), [U3]

$$
\begin{aligned}
& i(K) L^{\prime \natural}=i(K) \cdot L^{\natural} \cdot L^{\prime \natural} \simeq i(K) \cdot L^{\natural} \bigotimes_{L^{\prime}} L^{\prime \natural}=\mathscr{K} \bigotimes_{L^{4}} L^{\prime \natural}, \\
& i(L) L^{\prime k}=i(L) \cdot L^{\natural} \cdot L^{\prime \natural} \simeq i(L) \cdot L^{\natural} \bigotimes_{L^{\prime}} L^{\prime \natural}=\mathscr{L} \bigotimes_{L^{\prime}} L^{\prime \natural} \text {, } \\
& \mathscr{L} \cdot L^{\prime \prime} \simeq \mathscr{L} \otimes_{L^{\prime}} L^{\prime \prime} .
\end{aligned}
$$

To introduce the partial differential field $L^{\#}$ we take a transcendence basis

$$
\left\{u_{1}, u_{2}, \ldots, u_{n}\right\}
$$

of the extension $L^{\natural} / K^{\natural}$. So

$$
\left\{u_{1}, u_{2}, \ldots, u_{n}\right\}
$$

is also a transcendence basis of $L^{\prime \prime} / K_{2}^{\natural}$ and thus the partial differential field structure $L^{\prime \prime \prime}$ is defined on $L^{\prime \prime}$. So since $L$ is a field, to prove that the inclusion $i(L) L^{\#} \subset \mathscr{L}$ is in fact an equality, it is sufficient to show that the natural morphism

$$
L^{\natural} \otimes_{L^{\prime}} L^{\prime \prime} \simeq i(L) \cdot L^{\prime \prime} \rightarrow \mathscr{L} \cdot L^{\prime \prime} \simeq \mathscr{L} \otimes_{L^{\prime}} L^{\prime \prime}
$$

is an isomorphism

Since we have a $K_{2}$-valued point of $Y^{\natural}=\operatorname{Spec} A^{\natural}$, we have a morphism $A^{\natural}$ $\rightarrow K_{2}^{\natural}$ of abstract $K_{2}^{\natural}$-algebras, the isomorphism

$$
\text { Diff-hom }{ }_{K}\left(A, K_{2}^{\natural}[[t]]\right) \simeq \operatorname{Hom}_{K^{\prime}}\left(A^{\natural}, K_{2}^{\natural}\right)
$$

of Proposition (1.4), [U3] gives us a $\left(K_{2}^{\natural}[[t]], d / d t\right)$-valued point

$$
f_{1}: \operatorname{Spec} K_{2}^{\natural}[[t]] \rightarrow Y
$$

of the scheme $Y$ with derivation and hence a $\left(K_{2}^{\natural}[[t]], d / d t\right)$-valued point

$$
f_{2}: \operatorname{Spec} K_{2}^{\natural}[[t]] \rightarrow X
$$

of the scheme $X$ with derivation. Consequently we get a $\left(L^{\prime \prime}[[t]], d / d t\right)$-valued 
point

$$
f_{3}: \operatorname{Spec} L^{\prime \natural}[[t]] \rightarrow X
$$

of the scheme $X$ with derivation by composing the morphism $f_{2}$ with the inclusion $K_{2}^{\natural}[[t]] \hookrightarrow L^{\prime q}[[t]]$. Since the universal Taylor morphism $i: L \rightarrow L^{\natural}[[t]]$ composed with the inclusion $L^{\natural}[[t]] \hookrightarrow L^{\prime \natural}[[t]]$ defines another $\left(L^{\prime \natural}[[t]]\right.$, $d / d t)$-valued point

$$
f_{0}: \operatorname{Spec} L^{\prime \natural}[[t]] \rightarrow X
$$

of the scheme $X$ with derivation. It follows from (3.15) that there exists a point

$$
g \in G\left(C_{L^{\prime \prime}[[t]]}\right)=G\left(L^{\prime k}\right)
$$

such that

$$
g \cdot f_{0}=f_{3}, \quad f_{0}=g^{-1} \cdot f_{3} .
$$

In fact by $(3.15)$ the $K$-differential morphism

$$
\left(f_{0}, f_{3}\right): \operatorname{Spec} L^{\prime \natural}[[t]] \rightarrow X \times_{K} X
$$

gives a $K$-morphism

$$
\operatorname{Spec} L^{\prime q}[[t]] \rightarrow G \times_{C} X
$$

and hence by composing with the projection to the first factor, a differential morphism

$$
g_{0}: \operatorname{Spec} L^{\prime \natural}[[t]] \rightarrow G
$$

Since $G$ is a scheme with trivial derivation, the differential morphism $g_{0}$ factors through a Spec $C_{L^{\prime \prime}[[t]]}$-valued point yielding a morphism

$$
g_{0}: \operatorname{Spec} C_{L^{\prime \prime}[[t]]}=\operatorname{Spec} L^{\prime^{\natural}} \rightarrow G
$$

of schemes. We translate (3.16) into the language of rings. To this end let $Y_{j}(t)$ be the image of $y_{j}$ by the universal Taylor morphism $i: L \rightarrow L^{\natural}[[t]]\left(\subset L^{\prime q}[[t]]\right)$ and $Z_{j}(t) \in K_{2}^{\natural}[[t]] \subset L^{\prime \natural}[[t]]$ be the image of $y_{j}$ by $f_{3}$. Then (3.16) shows

$$
L^{\prime \prime}\left(i(K), Y_{1}, Y_{2}, \ldots, Y_{m}\right)=L^{\prime \prime}\left(i(K), Z_{1}, Z_{2}, \ldots, Z_{m}\right)
$$

in $L^{\prime \natural}[[t]]$. Since the $Z_{j}(1 \leq j \leq m)$ are in $K_{2}[[t]]$,

$$
\partial Z_{j} / \partial u_{l}=0 \text { for } 1 \leq l \leq n
$$

and 


$$
i(K)\left(Z_{1}, Z_{2}, \ldots, Z_{m}\right)
$$

is closed under the derivation $d / d t$, the field

$$
L^{\prime \prime}\left(i(K), Z_{1}, Z_{2}, \ldots, Z_{m}\right)
$$

is closed under the derivation $d / d t$. Thus the right side of (3.17) is closed under

$$
\left\{d / d t, \partial / \partial u_{1}, \partial / \partial u_{2}, \ldots, \partial / \partial u_{n}\right\}
$$

and hence so is the left side. The left side of $(3.17)$ is $L^{\prime *} . i(L)$. So $L^{\prime \prime} . i(L)$ is closed under

$$
\left\{d / d t, \partial / \partial u_{1}, \partial / \partial u_{2}, \ldots, \partial / \partial u_{n}\right\}
$$

Therefore we have

$$
\mathscr{L} \cdot L^{\prime \prime}=L^{\prime \#} \cdot i(L)
$$

in $L^{\prime \prime}[[t]]\left[t^{-1}\right]$ by definition of $\mathscr{L}$, which is the

$$
\left\{d / d t, \partial / \partial u_{1}, \partial / \partial u_{2}, \ldots, \partial / \partial u_{n}\right\} \text {-invariant }
$$

subfield of $L^{\#}[[t]]\left[t^{-1}\right]$ generated by $L^{\#}$ and $i(L)$. Since

$$
\mathscr{K}=i(K) \cdot L^{\#} \simeq \mathbf{Q}\left(i(K) \bigotimes_{C} L^{\#}\right),
$$

we have thus proved

$$
\mathscr{L}=i(L) \cdot L^{\#} \simeq \mathbf{Q}\left(i(L) \otimes_{C} L^{\#}\right)
$$

so that we have

$$
\mathscr{L} \simeq L \bigotimes_{K} \mathscr{K}
$$

Hence $X \otimes_{K} \mathscr{K}$ is a model of the differential field extension $\mathscr{L} / \mathscr{K}$, which is a principal homogeneous space of $G_{K} \otimes \mathscr{K}$. Namely we have an isomorphism

$$
G_{\mathscr{K}} \times_{\mathscr{K}} X_{\mathscr{K}} \stackrel{\widetilde{\rightarrow}}{\rightarrow} X_{\mathscr{K}} \times_{\mathscr{K}} X_{\mathscr{K}} \quad(g, x) \mapsto(g x, x)
$$

of schemes with a set of derivations

$$
\left\{d / d t, \partial / \partial u_{1}, \partial / \partial u_{2}, \ldots, \partial / \partial u_{n}\right\}_{\text {-invariant. }}
$$

Since $C_{\mathscr{L}}=C_{\mathscr{K}}=K_{1}^{\natural}, \mathscr{L} / \mathscr{K}$ is a strongly normal extension with Galois group $G_{K_{1}}$ by Theorem (3.10) of [U3] (only ordinary case is treated there but the result is easily extended to partial case). 


\section{\$4. Functors $\mathscr{F}_{\mathscr{L} / \mathcal{K}}$ and $\mathfrak{F}_{\mathscr{L} / \mathcal{K}}$}

Let $L / K$ be an ordinary differential field extension satisfying the condition (F.C) as in $\S 3$. We have introduced the differential field $\mathscr{K}$ and the differential field extension $\mathscr{L} / \mathcal{K}$ in $\S 3$. They are differential subfield of $L^{\natural}[[w, t]]\left[t^{-1}\right]$. For an $L^{\natural}$-algebra $A$ we denote by

$$
\operatorname{Diff}-\operatorname{hom}_{K}\left(\mathscr{L}, A[[w, t]]\left[t^{-1}\right]\right)
$$

the set of all differential morphisms

$$
f: \mathscr{L} \rightarrow A[[w, t]]\left[t^{-1}\right]
$$

that coincide with the morphism

$$
\iota: \mathscr{L} \rightarrow L^{\natural}[[w, t]]\left[t^{-1}\right]
$$

in (3.4.2) when restricted to the differential subfield $\mathscr{K}$. Namely the diagram

$$
\begin{array}{ccc}
\mathscr{L} & \stackrel{f}{\rightarrow} & A[[w, t]]\left[t^{-1}\right] \\
\uparrow & \uparrow \\
\mathscr{K} & \rightarrow & L^{\natural}[[w, t]]\left[t^{-1}\right]
\end{array}
$$

is commutative, where the lower horizontal arrow is the canonical morphism $\left.\iota\right|_{\mathscr{K}}$ and the left vertical arrow is the inclusion, and the right vertical arrow is induced from the structure morphism $L^{\natural} \rightarrow A$ of the $L^{\natural}$-algebra $A$. We say that

$$
f \in \operatorname{Diff}-\operatorname{hom}_{K}\left(\mathscr{L}, A[[w, t]]\left[t^{-1}\right]\right)
$$

is an infinitesimal deformation of the canonical morphism

$$
\mathscr{L} \rightarrow L^{\natural}[[w, t]]\left[t^{-1}\right]
$$

if the reduction of $f$ modulo the nilpotent radical $N(A)$ of $A$ coincides with the morphism $c$ in (3.4.2), i.e. if the diagram

$$
\begin{array}{ccc}
\mathscr{L} & \rightarrow & L^{\natural}[[w, t]]\left[t^{-1}\right] \\
\downarrow & & \downarrow \\
A[[w, t]]\left[t^{-1}\right] & \rightarrow & A / N(A)[[w, t]]\left[t^{-1}\right]
\end{array}
$$

is commutative. Here the upper horizontal arrow is the morphism (3.4.2), the lower horizontal arrow is the reduction modulo $N(A)$ of coefficients and the right vertical arrow is induced from the composite morphism

$$
L^{\natural} \rightarrow A \rightarrow A / N(A)
$$


of the structure morphism $L^{\natural} \rightarrow A$ and the reduction $A \rightarrow A / N(A)$. As in our preceding paper [U3], we introduce a functor

$$
\mathscr{F}_{\mathscr{L} / \mathscr{K}}:\left(\operatorname{Alg} / L^{\natural}\right) \rightarrow(\text { Set })
$$

by

$$
\mathscr{F}_{\mathscr{L} / \mathcal{K}}(A)=\left\{f \in \operatorname{Diff}_{-\operatorname{hom}_{K}}\left(\mathscr{L}, A[[w, t]]\left[t^{-1}\right]\right) \mid f\right. \text { is an infinitesimal }
$$

deformation of the canonical morphism $\iota: \mathscr{L} \rightarrow L^{\natural}[[w, t]]\left[t^{-1}\right]$ in (3.4.2)\}.

The definition of the functor $\mathscr{F}_{\mathscr{L} / \mathcal{K}}$ looks dependent on the choice of the transcendence basis

$$
\left\{u_{1}, u_{2}, \ldots, u_{n}\right\}
$$

of $L^{\natural} / K^{\natural}$.

PROPOSITION (4.1). The functor $\mathscr{F}_{\mathscr{L} / \mathscr{K}}$ is independent of the choice of a transcendence basis of $L^{\natural} / K^{\natural}$.

Proof. Let

$$
\left\{u_{1}, u_{2}, \ldots, u_{n}\right\}, \quad\left\{v_{1}, v_{2}, \ldots, v_{n}\right\}
$$

be transcendence basis of $L^{\natural} / K^{k}$. The infinitesimal deformation functor $\mathscr{F}_{\mathscr{L} / \mathscr{K}}$ defined by using

$$
\left\{u_{1}, u_{2}, \ldots, u_{n}\right\}
$$

and

$$
\left\{v_{1}, v_{2}, \ldots, v_{n}\right\}
$$

are respectively denoted by $\mathscr{F}_{u}$ and $\mathscr{F}_{v}$. We show that the functors $\mathscr{F}_{u}, \mathscr{F}_{v}$ are mutually isomorphic. Using the notation of the Proof of Lemma (3.1), we have

$$
\partial / \partial u_{i}=\sum_{j=1}^{n} a_{i j} \partial / \partial v_{j}
$$

for $1 \leq i \leq n$ with $\left(a_{i j}\right) \in \mathrm{GL}_{n}(L)$. We denote the universal Taylor morphisms

$$
\begin{gathered}
\left(L^{\#},\left\{\partial / \partial u_{1}, \partial / \partial u_{2}, \ldots, \partial / \partial u_{n}\right\}\right) \rightarrow L^{\natural}\left[\left[w_{1}, w_{2}, \ldots, w_{n}\right]\right] \\
\left(L^{\#},\left\{\partial / \partial v_{1}, \partial / \partial v_{2}, \ldots, \partial / \partial v_{n}\right\}\right) \rightarrow L^{\natural}\left[\left[z_{1}, z_{2}, \ldots, z_{n}\right]\right]
\end{gathered}
$$

respectively by $i_{u}$ and $i_{v}$. We define an $L^{\natural}$-morphism 


$$
\varphi: L^{\natural}\left[\left[z_{1}, z_{2}, \ldots, z_{n}\right]\right] \rightarrow L^{\natural}\left[\left[w_{1}, w_{2}, \ldots, w_{n}\right]\right]
$$

by

$$
\varphi\left(z_{i}\right)=i_{u}\left(v_{i}\right)-v_{i}
$$

for $1 \leq i \leq n$ so that the diagram

$$
\begin{array}{ccc}
L \stackrel{{ }^{{ }_{v}}}{\longrightarrow} & L^{\natural}\left[\left[z_{1}, z_{2}, \ldots, z_{n}\right]\right] \\
\| & \downarrow \varphi \\
L & \stackrel{{ }^{i_{u}}}{\longrightarrow} & L^{\natural}\left[\left[w_{1}, w_{2}, \ldots, w_{n}\right]\right]
\end{array}
$$

is commutative. If we define an $L^{\natural}$-morphism

$$
\psi: L^{\natural}[[w]] \rightarrow L^{\natural}[[z]]
$$

by

$$
\phi\left(w_{i}\right)=i_{v}\left(u_{i}\right)-u_{i}
$$

then

$$
\varphi^{\circ} \phi=\mathrm{Id}, \phi^{\circ} \varphi=\mathrm{Id}
$$

by the commutative diagram (4.2). Hence the morphism $\varphi$ is an isomorphism. Moreover if we identify

$$
\partial / \partial u_{i} \quad \text { with } \sum_{j=1}^{n} a_{i j} \partial / \partial v_{j}(1 \leq i \leq n)
$$

and

$$
\partial / \partial w_{\imath} \quad \text { with } \sum_{j=1}^{n} i_{v}\left(a_{i j}\right) \partial / \partial z_{j}(1 \leq i \leq n)
$$

then the morphism in (4.2) commute with the derivations. For an $L^{\natural}$-algebra $A$, the morphism $\varphi$ induces an $A$-isomorphism

$$
\varphi_{A}: A[[z, t]]\left[t^{-1}\right] \rightarrow A[[w, t]]\left[t^{-1}\right]
$$

by

$$
\varphi_{A}(t)=t, \quad \varphi_{A}\left(z_{i}\right)=\varphi\left(z_{\imath}\right) \quad(1 \leq i \leq n)
$$

Now if

$$
f:\left(\mathscr{L},\left\{\partial / \partial t, \partial / \partial u_{1}, \partial / \partial u_{2}, \ldots, \partial / \partial u_{n}\right\}\right) \rightarrow A[[w, t]]\left[t^{-1}\right]
$$


is an infinitesimal deformation of the canonical morphism

$$
\left(\mathscr{L},\left\{\partial / \partial t, \partial / \partial u_{1}, \partial / \partial u_{2}, \ldots, \partial / \partial u_{n}\right\}\right) \rightarrow L^{\natural}[[w, t]]\left[t^{-1}\right],
$$

then

$$
\varphi \circ f:\left(\mathscr{L},\left\{\partial / \partial t, \partial / \partial v_{1}, \partial / \partial v_{2}, \ldots, \partial / \partial v_{n}\right\}\right) \rightarrow A[[z, t]]\left[t^{-1}\right]
$$

is an infinitesimal deformation of the canonical morphism

$$
\left(\mathscr{L},\left\{\partial / \partial t, \partial / \partial v_{1}, \partial / \partial v_{2}, \ldots, \partial / \partial v_{n}\right\}\right) \rightarrow L^{\natural}[[z, t]]\left[t^{-1}\right]
$$

and a mapping

$$
\mathscr{F}_{v}(A) \rightarrow \mathscr{F}_{u}(A), \quad f \mapsto \varphi^{\circ} f
$$

gives an isomorphism of the functors $\mathscr{F}_{v}$ and $\mathscr{F}_{u}$. This proves the proposition.

We can find a differential $K$-subalgebra $R$ of $L$ that is of finite type over $K$ as an abstract $K$-algebra such that $L$ is the quotient field of $R$ (cf. Lemma (1.5), [U3]). Let

$$
y_{1}, y_{2}, \ldots, y_{m} \in L
$$

be a generator of the $K^{\natural}$-algebra $R^{\natural}$ so that we have

$$
\left.K\left[y_{1}, y_{2}, \ldots, y_{m}\right]=R \quad K\left(y_{1}\right) y_{2}, \ldots, y_{m}\right)=L .
$$

Since $R$ is closed under the derivation, we have a system of differential equation

$$
y_{i}^{\prime}=f_{i}\left(y_{1}, y_{2}, \ldots, y_{m}\right) \quad 1 \leq i \leq m,
$$

$f_{i}\left(Z_{1}, Z_{2}, \ldots, Z_{m}\right)$ being polynomials with coefficients in $K$. As in $\S 3$, we may assume that $\left\{y_{1}, y_{2}, \ldots, y_{n}\right\} \quad(n \leq m)$ is a transcendence basis of $L / K$ and we use $\left\{y_{1}, y_{2}, \ldots, y_{n}\right\}$ to construct $\mathscr{K}$ and $\mathscr{L}$. Let

$$
i: L \rightarrow \mathscr{L} \rightarrow L^{\natural}[[w, t]]\left[t^{-1}\right]
$$

be the composite of the canonical morphisms. We denote $i\left(y_{i}\right)$ by $Y_{j}(w, t)$ for $1 \leq j \leq m$ and identify $L, \mathscr{L}$ with their images in $L^{\natural}[[w, t]]\left[t^{-1}\right]$. In particular $y$, and $Y_{j}(w, t)$ are identified. It follows from the construction of $\mathscr{L}$ that $Y_{j}(w, t)$ is in fact in $L^{\natural}[[w, t]]$ for $1 \leq j \leq m$. Now the system (4.3) of differential equations is translated into the system

$$
\partial Y_{j}(w, t) / \partial t=F_{j}\left(Y_{1}, Y_{2}, \ldots, Y_{m}\right) \quad 1 \leq j \leq m .
$$

Here the 


$$
F_{j}\left(V_{1}, Y_{2}, \ldots, V_{m}\right) \quad 1 \leq j \leq m
$$

are polynomials in the $V_{l}(1 \leq l \leq m)$ with coefficients in $i(K)$ which is a subalgebra of $L^{\natural}[[t]]$.

Lemma (4.5). Let

$$
f \in \mathscr{F}_{\mathscr{L} / \mathscr{K}}(A)
$$

be an infinitesimal deformation of the canonical morphism

$$
\mathscr{L} \rightarrow L^{\natural}[[w, t]]\left[t^{-1}\right]
$$

in (3.4.2). Then $f\left(Y_{j}\right)$ is in $A[[w, t]]$ for $1 \leq j \leq m$ and there exists

$$
\Phi=\left(\varphi_{1}(w), \varphi_{2}(w), \ldots, \varphi_{n}(w)\right) \in \Gamma_{n L}(A)
$$

such that we have

$$
f\left(Y_{j}\right)=Y_{j}(\Phi(w), t) \quad \text { for } 1 \leq j \leq m .
$$

Proof. It follows from the definition of an infinitesimal deformation

$$
f\left(Y_{\imath}\right)=Y_{\imath}+Z_{i} \quad \text { with } Z_{\imath} \in N(A)[[w, t]]\left[t^{-1}\right]
$$

for $1 \leq i \leq m$. We show

$$
Z_{i} \in N(A)[[w, t]]
$$

To this end we denote by $Z_{i-}$ the polar part of $Z_{\imath}$ (with respect to $t$ ) so that

$$
Z_{i+}=Z_{i}-Z_{i-}
$$

is the regular part of $Z_{i}$. Therefore we have

$$
Z_{i}=Z_{i-}+Z_{\imath+}, \quad Z_{\imath-} \in t^{-1} N(A)[[w]]\left[t^{-1}\right], \quad Z_{\imath+} \in N(A)[[w, t]]
$$

for $1 \leq i \leq m$. Since $f$ is a $K$-morphism of differential algebras,

$$
\left(f\left(Y_{1}\right), f\left(Y_{2}\right), \ldots, f\left(Y_{m}\right)\right)
$$

satisfies the system (4.4) of differential equations:

$$
\partial f\left(Y_{i}\right)(w, t) / \partial t=F_{i}\left(f\left(Y_{1}\right), f\left(Y_{2}\right), \ldots, f\left(Y_{m}\right)\right) \quad 1 \leq i \leq m .
$$

The coefficients of $F_{i}$ are in $i(K)$, hence in $K[[t]]$ and in particular regular. We have to show that $Z_{i-}=0$. Assume that $Z_{i-} \neq 0$. Then among the $Z_{i-}(1 \leq i$ $\leq m$ ), let $Z_{\jmath_{-}}$take a pole of the highest order $d \geq 1$. It follows from (4.6) that we 
have

$$
\begin{array}{r}
\partial\left(Y_{j}+Z_{j-}+Z_{j+}\right) / \partial t=F_{j}\left(Y_{1}+Z_{1-}+Z_{1+}, Y_{2}+Z_{2-}+Z_{2+}, \ldots\right. \\
\left.\ldots, Y_{m}+Z_{m-}+Z_{m+}\right) .
\end{array}
$$

We denote the ideal generated by the coefficients of $Z_{i-}(1 \leq i \leq m)$ by $I$. So $I \subset$ $N(A)$. Let us first assume $I^{2}=0$. Since $I^{2}=0$ and since the $F_{j}$ are polynomials with regular coefficients, the left side of (4.7) has a pole of order $d+1$. Whereas the order of the pole of the right side is at most $d$. This is a contradiction. So we have proved $Z_{i-}=0$ if $I^{2}=0$. If $I^{2} \neq 0$, then we work over $A / I^{2}$ instead of $A$ itself to conclude that the coefficients of $Z_{i-}(1 \leq i \leq m)$ are in $I^{2}$. So $I=I^{2}$ and consequently $I=I^{l}$ for every positive integer $l$. If the ideal $I$ is generated by a finite number of nilpotent elements, $I^{l}=0$ for a sufficiently large integer $l$. Therefore $I=0$ and hence $Z_{i-}=0$ for $1 \leq i \leq m$. If the ideal $I$ is not finitely generated, then we replace $A[[w, t]]\left[t^{-1}\right]$ by $A[[w, t]]\left[t^{-1}\right] /\left(w^{l}\right)$. The above argument shows that $Z_{i-} \equiv 0 \bmod w^{l}$ for every $l \in \mathbf{N}$ so that $Z_{t_{-}}=0$. So we have proved

$$
f\left(Y_{\imath}\right) \in A[[w, t]] \quad \text { for } 1 \leq i \leq m .
$$

Let us set in $L^{\natural}[[w, t]]\left[t^{-1}\right]$

$$
f\left(Y_{i}\right)(w, 0)-y_{i}^{\natural}=\varphi_{i}(w) \text { for } 1 \leq i \leq m
$$

which are in $L^{\natural}[[w]]$ and

$$
\Phi=\left(\varphi_{1}, \varphi_{2}, \ldots, \varphi_{n}\right) .
$$

Here we denote by $y_{i}{ }^{\natural}$ the image of $y_{i} \in L$ by the natural morphism

$$
L^{\natural} \rightarrow L^{\natural}[[w, t]]\left[t^{-1}\right] .
$$

Since $f$ is an infinitesimal deformation of the canonical morphism

$$
\mathscr{L} \rightarrow L^{\natural}[[w, t]]\left[t^{-1}\right]
$$

in (3.4.2), we have

$$
Y_{i}(w, t) \equiv f\left(Y_{i}(w, t)\right) \bmod N(A)[[w, t]] \text { for } 1 \leq i \leq m
$$

and hence

$$
Y_{i}(w, 0) \equiv f\left(Y_{i}(w, 0)\right) \quad \bmod N(A)[[w]] \text { for } 1 \leq i \leq m .
$$

Since we take $u_{i}=y_{i}$ for $1 \leq i \leq n$, we have

$$
Y_{i}(w, 0)=y_{i}^{\natural}+w_{i}
$$


by definition and consequently

$$
w_{i} \equiv \varphi_{i}(w) \bmod N(A)[[w]] \text { for } 1 \leq i \leq n
$$

by the congruence above. So

$$
\Phi=\left(\varphi_{1}(w), \varphi_{2}(w), \ldots, \varphi_{n}(w)\right)
$$

is an infinitesimal coordinate transformation, i.e. we have

$$
\Phi \in \Gamma_{n L}{ }^{\prime}(A) .
$$

Since

$$
L^{\natural}[[w, t]] \rightarrow A[[w, t]] \quad(w \mapsto \Phi(w), t \mapsto t)
$$

is a $\partial / \partial t$-differential $L^{\natural}$-morphism and since the coefficients of $F_{i}(1 \leq i$ $\leq m)$ are in $L^{\natural}[[t]]\left(\subset L^{\natural}[[w, t]]\left[t^{-1}\right]\right)$,

$$
\left(Y_{1}(\Phi(w), t), Y_{2}(\Phi(w), t), \ldots, Y_{m}(\Phi(w), t)\right)
$$

is also a solution of (4.4). Since the coefficients of the system (4.6) of differential equations are in $A[[w, t]]$, the solution

$$
\left(f\left(Y_{1}\right), f\left(Y_{2}\right), \ldots, f\left(Y_{m}\right)\right)
$$

is determined by the initial condition

$$
\left(f\left(Y_{1}\right)(w, 0), f\left(Y_{2}\right)(w, 0), \ldots, f\left(Y_{m}\right)(w, 0)\right) .
$$

To prove

$$
f\left(Y_{i}(w, t)\right)=Y_{i}(\Phi(w), t) \quad \text { for } 1 \leq i \leq m,
$$

we have to show that their initial conditions coincide:

$$
f\left(Y_{\imath}\right)(w, 0)=Y_{i}(\Phi(w), 0) \text { for } 1 \leq i \leq m .
$$

In fact we have

$$
f\left(Y_{i}\right)(w, 0)=Y_{i}(\Phi(w), 0) \quad \text { for } 1 \leq i \leq n
$$

by definition. To see

$$
f\left(Y_{i}\right)(w, 0)=Y_{i}(\Phi(w), 0) \text { for } n+1 \leq i \leq m,
$$

we set

$$
L^{\prime}=i(L)=i(K)\left(Y_{1}, Y_{2}, \ldots, Y_{m}\right)
$$


which is a subfield of the ring $L^{\natural}[[w, t]]$. Identifying the field

$$
i(K)\left(\subset L^{\prime} \subset L^{\natural}[[w, t]]\right)
$$

with $K^{\natural} \subset A[[w]]$, we define two $K$-algebra morphisms

$$
h_{1}, h_{2}: L^{\prime} \rightarrow A[[w]]
$$

respectively by

$$
h_{1}\left(Y_{i}(w, t)\right)=f\left(Y_{i}\right)(w, 0) \quad \text { and } \quad h_{2}\left(Y_{\imath}(w, t)\right)=Y_{i}(\Phi(w), 0)
$$

for $1 \leq i \leq m$. Since the morphism

$$
h_{1}: L^{\prime} \rightarrow A[[w]]
$$

is the conposite of $K$-algebra morphism

$$
\left.f\right|_{L^{\prime}}: L^{\prime} \rightarrow A[[w, t]]
$$

and the $A[[w]]$-algebra morphism

$$
A[[w, t]] \rightarrow A[[w]]
$$

of substituting $w=0$, the morphism

$$
h_{1}: L^{\prime} \rightarrow A[[w]]
$$

is a well-defined $K$-algebra morphism. Similarly

$$
h_{2}: L^{\prime} \rightarrow A[[w]]
$$

is the composite morphism of the $L^{\natural}$-algebra morphism

$$
L^{\prime} \subset L^{\natural}[[w, t]] \rightarrow L^{\natural}[[w]]
$$

of substituting $t=0$ and the $L^{\natural}$-algebra morphism

$$
L^{\natural}[[w]] \rightarrow L[[w]]
$$

of substituting $w=\Phi(w)$. We have to show

Lemma (4.9). The morphisms

$$
h_{1}, h_{2}: L^{\prime} \rightarrow A[[w]]
$$

coincide.

Proof of Lemma. Let us set 


$$
L_{0}=i\left(K\left(y_{1}, y_{2}, \ldots, y_{n}\right)\right)=i(K)\left(Y_{1}, Y_{2}, \ldots, Y_{n}\right)
$$

which is a subfield of $L^{\prime}$. Then $h_{1}$ and $h_{2}$ coincide on $L_{0}$ by (4.8). We show $h_{1}(s)=h_{2}(s)$ for every $s \in L^{\prime}$. Let

$$
s^{l}+a_{1} s^{l-1}+\cdots+a_{l}=0 \quad\left(a_{1}, a_{2}, \ldots, a_{l} \in L_{0}\right)
$$

be the minimal polynomial of $s$ over $L_{0}$. It follows from the definition of infinitesimal deformation that every coefficient of the power series $h_{1}(s)-h_{2}(s) \in$ $A[[w]]$ is nilpotent. We first prove that the constant term of the power series $h_{1}(s)-h_{2}(s)$ is equal to 0 . Composing the morphisms

$$
h_{1}, h_{2}: L^{\prime} \rightarrow A[[w]]
$$

wiht the morphism

$$
A[[w]] \rightarrow A
$$

of evaluation at $w=0$, we get two $K$-algebra morphisms

$$
\bar{h}_{1}, \bar{h}_{2}: L^{\prime} \rightarrow A
$$

such that $\bar{h}_{1}$ and $\bar{h}_{2}$ coincide on $L_{0}$. The constant term of $h_{1}(s)-h_{2}(s)$ is $\bar{h}_{1}(s)$, $\bar{h}_{2}(s)$ which we denote by $\alpha$. By (4.10) we have

$$
\bar{h}_{1}(s)^{l}+\bar{h}_{1}\left(a_{1}\right) \bar{h}_{1}(s)^{l-1}+\cdots+\bar{h}_{1}\left(a_{l}\right)=0 .
$$

Substituting $\bar{h}_{1}(s)=\bar{h}_{2}(s)+\alpha$ in (4.11), we get

$$
\left(\bar{h}_{2}(s)+\alpha\right)^{l}+\bar{h}_{1}\left(a_{1}\right)\left(\bar{h}_{2}(s)+\alpha\right)^{l-1}+\cdots+\bar{h}_{1}\left(a_{1}\right)=0 .
$$

Since $\bar{h}_{1}$ coincides with $\bar{h}_{2}$ on $L_{0}$, it follows from (4.12)

$$
\left(\bar{h}_{2}(s)+\alpha\right)^{l}+\bar{h}_{2}\left(a_{1}\right)\left(\bar{h}_{2}(s)+\alpha\right)^{l-1}+\cdots+\bar{h}_{2}\left(a_{1}\right)=0 .
$$

Applying $\bar{h}_{2}$ to $(4.10)$, we have

$$
\bar{h}_{2}(s)^{l}+\bar{h}_{2}\left(a_{1}\right) \bar{h}_{2}(s)^{l-1}+\cdots+\bar{h}_{2}\left(a_{l}\right)=0 .
$$

If we assume here that $\alpha^{2}=0$, then it follows from (4.13) and (4.14) that

$$
G^{\prime}\left(\bar{h}_{2}(s)\right) \alpha=0,
$$

where we denote the polynomial

$$
x^{l}+\bar{h}_{2}\left(a_{1}\right) x^{l-1}+\cdots+\bar{h}_{2}\left(a_{l}\right)
$$

by $G(x)$ and $d G / d x$ by $G^{\prime}(x)$. Since we are in characteristic $0, \bar{h}_{2}(s)$ is a simple root of the characteristic polynomial, $G^{\prime}\left(\bar{h}_{2}(s)\right)$ is a non-zero element of a field 
$\bar{h}_{2}(L)$ and hence is a unit in $A$. Therefore $\alpha=0$. If $\alpha^{2} \neq 0$, then the argument of proving $Z_{i-}=0$ in the proof of Lemma (4.5) shows that $\alpha=0$. By the same argument we can show that for the reductions

$$
\tilde{h}_{1}, \tilde{h}_{2}: L^{\prime} \rightarrow A[[w]] \rightarrow A[[w]] /(w)^{k}
$$

modulo

$$
(w)^{k}=\left(w_{1}, w_{2}, \ldots, w_{n}\right)^{k}
$$

of the morphisms $h_{1}, h_{2}$, we have $\tilde{h}_{1}(s)=\tilde{h}_{2}(s)$ for every non-negative integer $k$. So $h_{1}(s)=h_{2}(s)$. This is what we had so show.

Remark. In the argument of proving $Z_{i-}=0$, the ideal $I$ should be finitely generated. Since $h_{1}(s)-h_{2}(s)$ has infinitely many coefficients, we have to consider the reductions modulo $(w)^{l}$ of the morphisms $h_{1}, h_{2}$.

$\mathscr{L}$ and $\mathscr{K}$ are differential algebras with a set

$$
\left\{\partial / \partial u_{1}, \partial / \partial u_{2}, \ldots, \partial / \partial u_{n}, \partial / \partial t\right\}
$$

of derivations. So we can consider the universal Taylor morphisms

$$
\mathscr{L} \rightarrow\left(\mathscr{L}^{\natural}[[w, T]],\left\{\partial / \partial w_{1}, \partial / \partial w_{2}, \ldots, \partial / \partial w_{n}, \partial / \partial T\right\}\right)
$$

and

$$
\mathscr{K} \rightarrow\left(\mathscr{K}^{\natural}[[w, T]],\left\{\partial / \partial w_{1}, \partial / \partial w_{2}, \ldots, \partial / \partial w_{n}, \partial / \partial T\right\}\right) .
$$

For example the universal Taylor morphism sends an element $a \in \mathscr{L}$ to

$$
\sum_{\alpha=\left(\alpha_{1}, \alpha_{2}, \ldots, \alpha_{n+1} \in \mathbf{N}^{n+1}\right.} \frac{1}{\alpha !} \frac{\partial^{|\alpha|}}{\partial^{\alpha_{1}} u_{1} \partial^{\alpha_{2}} u_{2} \ldots \partial^{\alpha_{n}} u_{n} \partial^{\alpha_{n+1}} t} w_{1}^{\alpha_{1}} w_{2}^{\alpha_{2}} \cdots w_{n}^{\alpha_{n}} T^{\alpha_{n+1}},
$$

which is an element of the power series ring $\mathscr{L}^{\natural}[[w, T]]$. For an $\mathscr{L}^{\natural}$-algebra $A$ we denote by

$$
\operatorname{Diff}_{-\operatorname{hom}_{\mathscr{K}}}(\mathscr{L}, A[[w, t]])
$$

the set of all differential morphisms

$$
f: \mathscr{L} \rightarrow A[[w, t]]
$$

such that the restriction to $\mathscr{K}$ of $f$ coincides with the universal Taylor morphism

$$
\mathscr{K} \rightarrow \mathscr{K}^{\natural}[[w, t]] \rightarrow \mathscr{L}^{\natural}[[w, t]] .
$$

Namely the diagram 


$$
\begin{array}{ccc}
\mathscr{L} & \stackrel{f}{\rightarrow} & A[[w, t]] \\
\uparrow & & \uparrow \\
\mathscr{K} & \rightarrow & \mathscr{L}^{\natural}[[w, t]]
\end{array}
$$

is commutative, where the lower horizontal arrow is the universal Taylor morph ism, the left vertical arrow is the inclusion map, and the right vertical arrow is induced from the structure morphism $\mathscr{L}^{\natural} \rightarrow A$ of the $\mathscr{L}^{\natural}$-algebra $A$. We say that

$$
f \in \operatorname{Diff}-\operatorname{hom}_{K}(\mathscr{L}, A[[w, t]])
$$

is an infinitesimal deformation of the universal Taylor morphism

$$
\mathscr{L} \rightarrow \mathscr{L}^{\natural}[[w, t]]
$$

if the reduction of $f$ modulo the nilpotent radical $N(A)$ of $A$ coincides with the in dentity, i.e. if the diagram

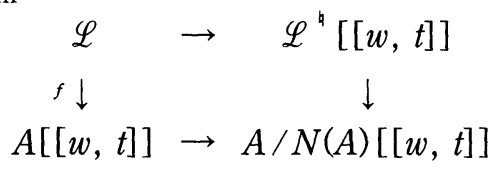

is commutative. Here the upper horizontal arrow is the universal Taylor morphism, the lower horizontal arrow is the reduction modulo $N(A)$ of coefficients and the right vertical arrow is induced from the composite morphism

$$
\mathscr{L}^{\natural} \rightarrow A \rightarrow A / N(A)
$$

of the structure morphism $\mathscr{L}^{\natural} \rightarrow A$ and the reduction $A \rightarrow A / N(A)$. Similarly as we defined the functor $\mathscr{F}_{\mathscr{L} / \mathscr{K}}$, we introduce a functor

$$
\mathfrak{F}_{\mathscr{L} / \mathscr{K}}:\left(\operatorname{Alg} / \mathscr{L}^{\natural}\right) \rightarrow(\text { Set })
$$

by

$$
\begin{gathered}
\mathfrak{F}_{\mathscr{L} / \mathcal{K}}(A)=\left\{f \in \operatorname{Diff}^{-\operatorname{hom}_{K}}(\mathscr{L}, A[[w, t]]) \mid f\right. \text { is an infinitesimal deformation of } \\
\text { the universal Taylor morphism } \left.\mathscr{L} \rightarrow \mathscr{L}^{\natural}[[w, t]]\right\} .
\end{gathered}
$$

Then the argument of the proof of Proposition (4.1) shows that the definition of the functor $\mathfrak{F}_{\mathscr{L} / \mathscr{K}}$ is independent of the choice of the transcendence basis

$$
\left\{u_{1}, u_{2}, \ldots, u_{n}\right\}
$$

of $L^{\natural} / K^{\natural}$. The functor $\mathfrak{F}_{\mathscr{L} / \mathscr{H}}$ is more natural than $\mathscr{F}_{\mathscr{L} / \mathscr{K}}$. It has however a disadvantage that the reference field $\mathscr{L}^{\natural}$ is in general very big. We denote the image of $y_{i}$ under the universal Taylor morphism by $Y_{i}(w, T)$ for $1 \leq i \leq m$. The argument of the proof of Lemma (4.5) allows us to prove 
Lemma (4.15). Let $f \in \mathfrak{F}_{\mathscr{L} / \mathcal{K}}(A)$ for an $\mathscr{L}^{\natural}$-algebra $A$. Then there exists

$$
\Phi=\left(\varphi_{1}(w), \varphi_{2}(w), \ldots, \varphi_{n}(w)\right) \in \Gamma_{n \mathscr{L}^{*}}(A)
$$

such that we have

$$
f\left(Y_{i}\right)=Y_{i}(\Phi(w), T) \quad \text { for } 1 \leq i \leq m
$$

In fact since we are working in the power series ring instead of the Laurent series ring, it is easier. In the proof of Lemma (4.5), using the notation there we had first to show that the infinitesimal deformations $f\left(Y_{\imath}\right)$ are in $A[[w, t]]$. But this condition is satisfied in the case of Lemma (4.15) by definition.

Let $L / K$ be an ordinary differential field extension and $K \subset M \subset L$ a differential intermediate field. We take a transcendence basis

$$
\left\{u_{1}, u_{2}, \ldots, u_{n^{\prime}}\right\}
$$

of $M^{\natural} / K^{\natural}$ and extend it to a transcendence basis

$$
\left\{u_{1}, u_{2}, \ldots, u_{n^{\prime}}, u_{n^{\prime}+1}, \ldots, u_{n}\right\}
$$

of $L^{\natural} / K^{\natural}$. So

$$
\left(M^{\natural},\left\{\partial / \partial u_{1}, \partial / \partial u_{2}, \ldots, \partial / \partial u_{n^{\prime}}\right\}\right)
$$

is a differential subalgebra of

$$
\left(L^{\natural},\left\{\partial / \partial u_{1}, \partial / \partial u_{2}, \ldots, \partial / \partial u_{n}\right\}\right) .
$$

By construction in $\S 3 \mathcal{M}=\mathscr{L}_{M / K}$ is a differential subfield of $M^{\#}[[t]]\left[t^{-1}\right]$ and $\mathscr{L}$ a differential subfield of $L^{\#}[[t]]\left[t^{-1}\right]$. So both $\mathscr{L}$ and $\mathcal{M}$ are differential subfields of $L^{\#}[[t]]\left[t^{-1}\right]$ and $\mathcal{M}$ is a differential subfield of $\mathscr{L}$. Let $A$ be an $\mathscr{L}^{\natural}$-algebra and

$$
f: \mathscr{L} \rightarrow A[[w, T]]
$$

be an infinitesimal deformation of the universal Taylor morphism

$$
\mathscr{L} \rightarrow \mathscr{L}^{\natural}[[w, T]] .
$$

By restriction $f$ induces an infinitesimal deformation

$$
\left.f\right|_{\mathcal{M}}: M \rightarrow A\left[\left[w_{1}, w_{2}, \ldots, w_{n}, T\right]\right]
$$

Since $\left.f\right|_{\mathcal{M}}$ is a differential morphism, it factors through the differential subalgebra

$$
A\left[\left[w_{1}, w_{2}, \ldots, w_{n^{\prime}}, T\right]\right]
$$


of

$$
A\left[\left[w_{1}, w_{2}, \ldots, w_{n}, T\right]\right] .
$$

Hence $\left.f\right|_{\mathcal{M}}$ is an infinitesimal deformation of the universal Taylor morphism

$$
\mu \rightarrow \mathcal{M}^{\natural}\left[\left[w_{1}, w_{2}, \ldots, w_{n^{\prime}}, T\right]\right] \rightarrow \mathscr{L}^{\natural}\left[\left[w_{1}, w_{2}, \ldots, w_{n^{\prime}}, T\right]\right] .
$$

If we denote the restriction of the functor

$$
\mathfrak{F}_{\mathcal{M} / \mathcal{K}}:\left(\operatorname{Alg} / M^{\natural}\right) \rightarrow(\text { Set })
$$

to the subcategory $\left(A \lg / \mathscr{L}^{\natural}\right)$ of $\left(A \lg / \mathcal{M}^{\natural}\right)$ by

$$
\mathfrak{F}_{\mathcal{M} \mathscr{K}} \otimes_{\mathcal{M}^{\prime}} \mathscr{L}^{\natural},
$$

we have a morphism

$$
\mathfrak{F}_{\mathscr{L} / \mathscr{K}} \rightarrow \mathfrak{F}_{\mathcal{M} \mathscr{H}} \otimes_{\mathcal{M}} \mathscr{L}^{\natural}
$$

of functors. Namely we define

$$
\mathfrak{F}_{\mathscr{L} / \mathcal{H}}(A) \rightarrow \mathfrak{F}_{\mathcal{M} / \mathcal{K}} \otimes_{\mathcal{M}} \cdot \mathscr{L}^{\natural}(A)=\mathfrak{F}_{\mathcal{M} / \mathcal{H}}(A)
$$

by sending

$$
f \in \mathfrak{F}_{\mathscr{L} / \mathscr{K}}(A) \text { to }\left.f\right|_{\mathcal{M}} \in \mathfrak{F}_{\mathcal{M} / \mathcal{K}}(A)
$$

for an $\mathscr{L}^{\natural}$-algebra $A$.

Proposition (4.16). For every $\mathscr{L}^{\natural}$-algebra $A$, the functorial morphism

$$
\mathfrak{F}_{\mathscr{L} / \mathcal{K}}(A) \rightarrow \mathfrak{F}_{\mathcal{M} / \mathcal{K}} \otimes_{\mathcal{M}^{*}} \mathscr{L}^{\natural}(A)=\mathfrak{F}_{\mathcal{M} / \mathcal{K}}(A)
$$

is surjective.

Proof. In Proposition (1.4) of [U3] we have proved that for an ordinary differential ring the universal Taylor morphism is universal among the Taylor morphisms. The same argument allows us to prove it for general case. Let $f \in$ $\mathfrak{F}_{\mathcal{M} / \mathcal{K}}(A)$ and $g \in \operatorname{Hom}_{K}\left(\mathcal{M}^{\natural}, A\right)$ be the morphism corresponding to $f$ by the isomorphism

$$
\operatorname{Diff}_{-\operatorname{hom}_{\mathscr{K}}}(\mathcal{M}, A[[w, T]]) \simeq \operatorname{Hom}_{\mathscr{K}^{*}}\left(\mathcal{M}^{\natural}, A\right),
$$

which is the universality of the universal Taylor morphism. Then $g: \mathcal{M}^{\natural} \rightarrow A$ is a $\mathscr{K}^{\natural}$-infinitesimal deformation of the inclusion $\mathcal{M}^{\natural} \rightarrow \mathscr{L}$. Namely the reduction

$$
M^{\natural} \rightarrow A \rightarrow A / N(A)
$$


of $g \bmod N(A)$ is the inclusion map

$$
M^{\natural} \rightarrow \mathscr{L}^{\natural}(\rightarrow A \rightarrow A / N(A)) .
$$

It is sufficient to show that we can extend the corresponding morphism $g: \mathcal{M}^{\natural} \rightarrow$ $A$, which is an infinitesimal deformation of the inclusion morphism $\mathcal{M}^{\natural} \hookrightarrow \mathscr{L}^{\natural}$, to an infinitesimal deformation of the identity morphism Id: $\mathscr{L}^{\natural} \rightarrow \mathscr{L}^{\natural}$. To this end it is sufficient to prove by Zorn's lemma the following

Lemma (4.17). Let $\mathcal{M}^{\prime}$ be an abstract intermediate field between $\mathcal{M}^{\natural}$ and $\mathscr{L}^{\natural}$. If the infinitesimal deformation $g: M^{\natural} \rightarrow A$ is extended to an infinitesimal deformation $g^{\prime}: \mathscr{M}^{\prime} \rightarrow A$ of the inclusion morphism $\mathscr{M}^{\prime} \hookrightarrow \mathscr{L}^{\natural}$, then for any element $u \in \mathscr{L}^{\natural}$, we can extend $g^{\prime}: M^{\prime} \rightarrow A$ to an infinitesimal deformation of the inclusion morphism $\mathcal{M}^{\prime}(u) \hookrightarrow \mathscr{L}^{\natural}$.

Proof of Lemma. Maybe this is well-known: A field extension in characteristic 0 is smooth. We briefly indicate here a proof for convenience of nonspecialists. If $u$ is transcendental over $\mathcal{M}^{\prime}$, then we take any element $v \in A$ (e.g. $v=u$ ) such that

$$
u \equiv v \bmod N(A) .
$$

So we get an infinitesimal deformation $h: \mathscr{M}^{\prime}[u] \rightarrow A$ of the inclusion morphism $\mathscr{M}^{\prime}[u] \hookrightarrow \mathscr{L}^{\natural}$. Then we can extend $h: \mathcal{M}^{\prime}[u] \rightarrow A$ to the quotient field $\mathcal{M}^{\prime}(u)$ so that we have an infinitesimal deformation $k: \mathcal{M}^{\prime}(u) \rightarrow A$. In fact for $0 \neq a \in$ $\mathscr{M}^{\prime}[u]$,

$$
h(a)=a+w=a\left(1+a^{-1} w\right)
$$

with $w \in N(A)$ and hence $a^{-1} w \in N(A)$, is invertible in $A$ :

$$
h(a)^{-1}=a^{-1}\left(1-a^{-1} w-\left(a^{-1} w\right)^{2}-\cdots\right) .
$$

If $u$ is algebraic over $\mathcal{M}^{\prime}$, then let

$$
F(u)=u^{m}+a_{1} u^{m-1}+a_{2} u^{m-2}+\cdots+a_{m}=0,
$$

$F(x)$ being the minimal polynomial of $u$ over $\mathcal{M}^{\prime}$. We have to show that there exists an element $v \in A$ such that $u \equiv v \bmod N(A)$ and such that

$$
v^{m}+g^{\prime}\left(a_{1}\right) v^{m-1}+g^{\prime}\left(a_{2}\right) v^{m-2}+\cdots+g^{\prime}\left(a_{m}\right)=0 .
$$

To solve (4.18) we may replace 


$$
\mathcal{M}^{\prime} \text { by } \mathcal{M}^{\natural}\left(a_{1}, a_{2}, \ldots, a_{m}\right)
$$

and

$$
A \text { by } \mathscr{L}^{\natural}\left[g^{\prime}\left(a_{1}\right), g^{\prime}\left(a_{2}\right), \ldots, g^{\prime}\left(a_{n}\right)\right] \text {, }
$$

which is a subring of $A$, so that the ring $A$ is of finite type over $\mathscr{L}^{k}$ and hence $N(A)$ is nilpotent. Say $N(A)^{s}=0$ for a positive integer $s$. We may assume that $N(A)^{2}=0$. For once we can solve (4.18) under the assumption that $N(A)^{2}=0$, then we can lift the canonical morphism

$$
\mathcal{M}^{\prime}[u] \rightarrow A / N(A),
$$

to an infinitesimal deformation

$$
h_{2}: \mathcal{M}^{\prime}[u] \rightarrow A / N(A)^{2}
$$

that extends the reduction

$$
\bar{g}_{2}^{\prime}: \mathscr{M}^{\prime} \rightarrow A \rightarrow A / N(A)^{2}
$$

of $g^{\prime} \bmod N(A)$, then to an infinitesimal deformation

$$
h_{3}: \mathcal{M}^{\prime}[u] \rightarrow A / N(A)^{3}
$$

that extends the reduction

$$
\bar{g}_{3}^{\prime}: \mathscr{M}^{\prime} \rightarrow A \rightarrow A / N(A)^{3}
$$

of $g^{\prime} \bmod N(A)^{3}$ and so on by succesive approximation. If we take $l \geq s$, then the reduction $\bar{g}_{l}^{\prime}$ of $g^{\prime} \bmod N(A)^{l}=0$ is $g^{\prime}$ itself and

$$
h_{l}: \mathcal{M}^{\prime}[u] \rightarrow A / N(A)^{s}=A
$$

is a desird extension of $g^{\prime}$. Now if $N(A)^{2}=0$, then setting

$$
g\left(a_{i}\right)=a_{i}+w_{i} \in A \quad \text { with } w_{i}^{2}=0 \quad \text { for } 1 \leq i \leq m,
$$

we look for $v=u+z$ with $z^{2}=0$ satisfying (4.18):

$$
(u+z)^{m}+\left(a_{1}+w_{1}\right)(u+z)^{m-1}+\cdots+\left(a_{m}+w_{m}\right)=0 .
$$

(4.19) is equivalent to

$$
F^{\prime}(u) z+w_{1} u^{m-1}+w_{2} u^{m-2}+\cdots+w_{m}=0 .
$$

Since we are in characteristic 0 and since $F$ is the minimal polynomial of $u$, we have $F^{\prime}(u) \neq 0$ and so $F^{\prime}(u)$ is invertible in $\mathscr{L}^{\natural}$ hence in $A$. Thus we can solve 


$$
z=-\left(w_{1} u^{m-1}+w_{2} u^{m-2}+\cdots+w_{m}\right) F^{\prime}(u)^{-1} .
$$

For the functor $\mathscr{F}$, similarly we have a morphism

$$
\mathscr{F}_{\mathscr{L} / \mathcal{K}} \rightarrow \mathscr{F}_{\mathcal{M} / \mathcal{K}} \otimes_{M} \text {, } L^{\natural}
$$

of functors on $\left(A \lg / L^{\natural}\right)$. We show in $\S 5$, Corollary $(5.12)$ that the morphism (4.21) is surjective. More precisely the functorial morphism

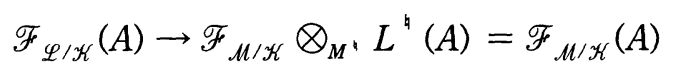

is surjective for all $\mathscr{L}^{\natural}$-algebra $A$. We do not know a direct proof of the surjectivity of (4.22).

\section{\$5. Infinitesimal Galois group}

We use the notations of $\S 4$. Let

$$
f: \mathscr{L} \rightarrow A[[w, t]]\left[t^{-1}\right]
$$

be an infinitesimal deformation of the canonical morphism

$$
\mathscr{L} \rightarrow L^{\natural}[[w, t]]\left[t^{-1}\right] .
$$

Lemma (5.1). The subalgebras $f(\mathscr{L})$ and $A[[w]]$ of $A[[w, t]]\left[t^{-1}\right]$ are linearly disjoint over $L^{\#}$.

Proof. Since the field of the constants of the differential field $(\mathscr{L}, \partial / \partial t)$, which is a differential subfield of $\left(L^{\#}[[t]]\left[t^{-1}\right], \partial / \partial t\right)$, is $L^{\#}$ and since $f$ is a $\mathscr{K}$-morphism, the field of the constants of $(f(\mathscr{L}), \partial / \partial t)$ is $L^{\#}$. Hence if we consider $A[[w, t]]\left[t^{-1}\right]$ as a differential algebra with derivation $\partial / \partial t$, then the lemma follows from Lemma (1.1) of [U3].

Corollary (5.2). The subalgebras $\mathscr{L}$ and $A[[w]]$ of $A[[w, t]]\left[t^{-1}\right]$ are linearly disjoint over $L^{\#}$.

Proof. This is a particular case of Lemma (5.1) if we take as $f$ the canonical morphism

$$
\mathscr{L} \rightarrow A[[w, t]]\left[t^{-1}\right]
$$


Lemma (5.3). The following conditions on an element $z$ of $\mathbf{Q}[\mathscr{L}, A[[w]]]$ are equivalent:

(1) $z$ is divisible by $w$ in $\mathbf{Q}[\mathscr{L}, A[[w]]]$;

(2) $z$ is divisible by $w$ in $A[[w, t]]\left[t^{-1}\right]$, which is an over-ring of $\mathbf{Q}[\mathscr{L}, A[[w]]]$.

Proof. Since the condition (1) evidently implies (2), we assume the condition (2) and prove (1). Let $z=z(w, t) \in \mathbf{Q}[\mathscr{L}, A[[w]]]$. It follows from Corollary (5.2)

$$
\mathbf{Q}[\mathscr{L}, A[[w]]] \simeq \mathscr{L} \bigotimes_{L^{\#}} A[[w]]
$$

So we can write

$$
z(w, t)=\sum_{i=1}^{\nu} a_{i}(w) z_{i}(w, t)
$$

with

$$
a_{\imath}(w) \in A[[w]]
$$

and

$$
z_{i}(w, t) \in \mathscr{L}
$$

for $1 \leq i \leq \nu$ such that the $z_{i}(w, t) \in \mathscr{L}$ are linearly independent over $L^{\#}$. It follows from (2) that there exists $z^{\prime} \in A[[w, t]]\left[t^{-1}\right]$ such that

$$
z(w, t)=\sum_{i=1}^{\nu} a_{\imath}(w) z_{\imath}(w, t)=w z^{\prime}(w, t)
$$

Differentiating successively by $t$, we get

$$
\sum_{i=1}^{\nu} a_{i}(w) \partial^{s} z_{\imath}(w, t) / \partial t^{s} \in w A[[w, t]]\left[t^{-1}\right]
$$

for $0 \leq s \leq \nu-1$. Since the $z_{i}(w, t)$ are linearly independent over $L^{\#}$, which is the field of the constants of the differential field $(\mathscr{L}, \partial / \partial t)$, the Wronskian

$$
\left|\delta^{s} z_{i}(w, t)\right|_{\substack{0 \leq s \leq \nu-1 \\ 1 \leq i \leq \nu}} \in \mathscr{L}
$$

is not equal to 0 . So the Wronskian is a unit in field $\mathscr{L}$ hence in $A[[w, t]]\left[t^{-1}\right]$. So considering the $a_{i}$ as unknowns, we can solve the linear equation (5.3.3) by Cramer's rule to conclude

$$
a_{\imath}(w) \in w A[[w, t]]\left[t^{-1}\right] \text { for } 1 \leq i \leq \nu .
$$


Hence $a_{i}(w) \in w A[[w]]$ for $1 \leq i \leq \nu$ and consequently

$$
z(w, t)=\sum_{i=1}^{\nu} a_{i}(w) z_{i}(w, t)
$$

is divisible by $w$ in $\mathbf{Q}[\mathscr{L}, A[[w]]]$. So the condition (1) is satisfied.

COROLlaRY (5.4) TO THE PROOF. Let $f$ be an infinitesimal deformation of the canonical morphism

$$
\mathscr{L} \rightarrow L^{\natural}[[w, t]]\left[t^{-1}\right]
$$

The following conditions on an element $\boldsymbol{z}$ of $\mathbf{Q}[f(\mathscr{L}), A[[w]]]$ are equivalent:

(1) $f(z)$ is divisible by $w$ in $\mathbf{Q}[f(\mathscr{L}), A[[w]]]$;

(2) $f(z)$ is divisible by $A[[w, t]]\left[t^{-1}\right]$, which is an over-ring of $\mathbf{Q}[f(\mathscr{L})$, $A[[w]]]$.

Let

$$
f: \mathscr{L} \rightarrow A[[w, t]]\left[t^{-1}\right]
$$

be an infinitesimal deformation of the canonical morphism

$$
f: \mathscr{L} \rightarrow \mathscr{L}[[w, t]]\left[t^{-1}\right] .
$$

Then if follows from Lemma (4.5) that there exists

$$
\Phi=\left(\varphi_{1}(w), \varphi_{2}(w), \ldots, \varphi_{n}(w)\right) \in \Gamma_{n L^{\prime}}(A)
$$

such that

$$
f\left(Y_{i}\right)=Y_{i}(\Phi(w), t) \quad \text { for } \quad 1 \leq i \leq m
$$

Let us set

$$
\Phi=\left(w_{1}+\varphi_{1}^{\prime}(w), w_{2}+\varphi_{2}^{\prime}(w), \ldots, w_{n}+\varphi_{n}^{\prime}(w)\right)
$$

and

$$
\Phi^{\prime}=\left(\varphi_{1}^{\prime}(w), \varphi_{2}^{\prime}(w), \ldots, \varphi_{n}^{\prime}(w)\right)
$$

so that

$$
\Phi=\mathrm{Id}+\Phi^{\prime}
$$

We denote 


$$
\Phi^{\prime l}=\varphi_{1}^{\prime l_{1}} \varphi_{2}^{\prime l_{2}} \cdots \varphi_{n}^{\prime l_{n}}
$$

for $l=\left(l_{1}, l_{2}, \ldots, l_{n}\right) \in \mathbf{N}^{n}$.

Lemma (5.5). For any positive number $\nu$, there exists a number $N$ such that if $l_{i}>N$ for every $1 \leq i \leq n$, then

$$
\Phi^{\prime l} \in(w)^{\nu}
$$

Proof. Lemma follows from the fact that the constant terms of the $\varphi_{\imath}(w)$ are nilpotent.

Lemma (5.6). We have a $\mathscr{K} \bigotimes_{L^{\#}} A[[w]]$-isomorphism

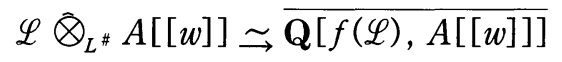

sending $z \in \mathscr{L}$ to $f(z)$. Here

$$
\mathscr{L} \bar{\bigotimes}_{L^{\#}} A[[w]]
$$

is the completion of

$$
\mathscr{L} \bigotimes_{L^{\#}} A[[w]]
$$

with respect to the $(1 \otimes w)$-adic topology and

$$
\overline{\mathbf{Q}[f(\mathscr{L}), A[[w]]]}
$$

is the closure of

$$
\mathbf{Q}[f(\mathscr{L}), A[[w]]]
$$

in $\mathbf{Q}[[w, t]]\left[t^{-1}\right]$ wiht respect to the $(w)$-adic topology.

Proof. By Lemma (5.1) we have a $\mathscr{K} \bigotimes_{L^{\sharp}} A[[w]]$-isomorphism

$$
\mathscr{L} \otimes_{L^{\#}} A[[w]] \underset{\rightarrow}{\sim} \mathbf{Q}[f(\mathscr{L}), A[[w]]] .
$$

Now the lemma follows Lemma (5.4)

LEMma (5.7). We have

$$
\overline{\mathbf{Q}[\mathscr{L}, A[[w]]]}=\overline{\mathbf{Q}[f(\mathscr{L}), A[[w]]]}
$$

in $A[[w, t]]\left[t^{-1}\right]$ 
Proof. We have

$$
f\left(Y_{i}\right)=Y_{i}(\Phi(w), t) \quad \text { for } \quad 1 \leq i \leq m .
$$

Since the $Y_{i}(w, t)$ are in

$$
L^{\natural}[[w, t]]\left[t^{-1}\right]
$$

and since

$$
\Phi=\mathrm{Id}+\Phi^{\prime} \quad \text { with } \Phi^{\prime}=\left(\varphi_{1}^{\prime}(w), \varphi_{2}^{\prime}(w), \ldots, \varphi_{n}^{\prime}(w)\right) \in(N(A)[[w]])^{n},
$$

it follows from the Taylor formula

$$
f\left(Y_{i}\right)=Y_{\imath}(\Phi(w), t)=Y_{i}\left(\operatorname{Id}+\Phi^{\prime}, t\right)=\sum_{l \in \mathbf{N}^{n}} \frac{1}{l !} \frac{\partial^{l} Y_{i}}{\partial w^{l}} \Phi^{\prime l}
$$

so that $f\left(Y_{i}\right) \in \overline{\mathbf{Q}[\mathscr{L}, A[[w]]]}$ by Lemma (5.5). So if we set $\mathscr{R}=L^{\#}\left\{Y_{1}, Y_{2}, \ldots\right.$, $\left.Y_{m}\right\}$, then

$$
f(R) \subset \overline{\mathbf{Q}[\mathscr{L}, A[[w]]]} .
$$

Let $0 \neq z \in R$. Then it follows from (5.7.1) and the proof of Lemma (5.5) that we can write $f(z)=z+v$, where $v$ is an element of the ideal of $A[[w, t]]\left[t^{-1}\right]$ generated by $N(A)[[w]]$ and

$$
f(z)^{-1}=(z+v)^{-1}=z^{-1}\left(1+z^{-1} v\right)^{-1} \in \overline{\mathbf{Q}[\mathscr{L}, A[[w]]]} .
$$

Therefore since $\mathscr{L}$ is the quotient field of $\mathscr{R}$,

$$
f(\mathscr{L}) \subset \overline{\mathbf{Q}[\mathscr{L}, A[[w]]]},
$$

hence

$$
\mathbf{Q}[f(\mathscr{L}), A[[w]]] \subset \overline{\mathbf{Q}[\mathscr{L}, A[[w]]]}
$$

and

$$
\overline{\mathbf{Q}[f(\mathscr{L}), A[[w]]]} \subset \overline{\mathbf{Q}[\mathscr{L}, A[[w]]]} .
$$

For the same reason, we get the opposite inclusion

$$
\overline{\mathbf{Q}[f(\mathscr{L}), A[[w]]]} \supset \overline{\mathbf{Q}[\mathscr{L}, A[[w]]]} .
$$

By Lemmas (5.6) and (5.7) we have $\mathscr{K} \hat{\bigotimes}_{L^{\sharp}} A[[w]]$-isomorphism

$$
\mathscr{L} \hat{\otimes}_{L^{\#}} A[[w]] \stackrel{\rightarrow}{\rightarrow} \overline{\mathbf{Q}[f(\mathscr{L}), A[[w]]]}=\overline{\mathbf{Q}[\mathscr{L}, A[[w]]]} \stackrel{\sim}{\rightarrow} \hat{\otimes}_{L^{\#}} A[[w]] .
$$

The above determined $\mathscr{K} \hat{\bigotimes}_{L^{*}} A[[w]]$-automorphism 


$$
\mathscr{L} \widehat{\otimes}_{L^{\sharp}} A[[w]] \stackrel{\rightarrow}{\mathscr{L}} \hat{\bigotimes}_{L^{\sharp}} A[[w]]
$$

of $\{\partial / \partial w, \partial / \partial t\}$-differential algebra $\mathscr{L} \hat{\otimes}_{L^{\sharp}} A[[w]]$ will be denoted by $f_{*}$. It follows from the construction that the composite morphism

$$
\mathscr{L} \rightarrow \mathscr{L} \hat{\otimes}_{L^{\sharp}} A[[w]] \rightarrow \mathscr{L} \hat{\otimes}_{L^{\sharp}} A[[w]] \simeq \mathbf{Q}[\mathscr{L}, A[[w]]]
$$

is $f$, where the first morphism is the canonical morphism $\mathscr{L} \rightarrow \mathscr{L} \otimes 1$, the second is $f_{*}$ and the last is the morphism in Lemma (5.6), $f$ in the lemma being the canonical morphism

Definition (5.8). We define a group functor

$$
\text { Inf-diff-bir }{ }_{K} L:\left(A l g / L^{\natural}\right) \rightarrow(G r p)
$$

by

Inf-diff-bir ${ }_{K} L(A)=\left\{f \mid f\right.$ is a differential $\mathscr{K} \tilde{\otimes}_{L^{\sharp}} A[[w]]$-automorphism of $\mathscr{L} \tilde{\otimes}_{L^{\sharp}} A[[w]]$ that is continuous with respect to the $(1 \otimes w)$-adic topology and congruent to the identity modulo $\left.\mathscr{L} \hat{\otimes}_{L^{\sharp}} N(A)[[w]]\right\}$

for an $L^{\natural}$-algebra $A$.

Lemma (5.9). The group functor

$$
\text { Inf-diff- } \mathbf{b i r}_{K} L:\left(A \lg / L^{\natural}\right) \rightarrow(G r p)
$$

is a Lie-Ritt functor.

Proof. We show that there exists an isomorphism

$$
\text { Inf-diff- } \text { bir }_{K} L \simeq \mathscr{F}_{\mathscr{L} / \mathcal{K}}
$$

of functors. In fact let $A$ be an $L^{\natural}$-algebra. By Lemma (5.6), $\mathscr{L} \bar{\otimes}_{L^{\sharp}} A[[w]]$ is isomorphic to $\overline{\mathbf{Q}[\mathscr{L}, A[[w]]]}$ which is a subalgebra of $A[[w, t]]\left[t^{-1}\right]$. So $f \in$ Inf-diff-bir $(A)$ defines an infinitesimal deformation

$$
\mathscr{L} \rightarrow \mathscr{L} \bar{\otimes}_{L^{\sharp}} A[[w]] \stackrel{f}{\rightarrow} \mathscr{L} \hat{\otimes}_{L^{\sharp}} A[[w]] \simeq \overline{\mathbf{Q}[\mathscr{L}, A[[w]]]} \subset A[[w, t]]\left[t^{-1}\right],
$$

where the first morphism

$$
\mathscr{L} \rightarrow \mathscr{L} \bar{\bigotimes}_{L^{\sharp}} A[[w]]
$$

is the canonical morphism of identifying $\mathscr{L}$ with $\mathscr{L} \otimes 1$. We denote this deformation by $f^{\prime}$. So we get a functorial map 


\section{Inf-diff-bir $(A) \gtrsim \mathscr{F}_{\mathscr{L} / \mathcal{K}}(A)$}

which sends an automorphism $f$ to $f^{\prime}$. We have defined above the functorial mapping

$$
\mathscr{F}_{\mathscr{L} / \mathscr{K}}(A) \rightarrow \operatorname{Inf}-\operatorname{diff}-\operatorname{bir}(A), \quad h \mapsto h_{*} \text {. }
$$

We have $\left(f_{*}\right)^{\prime}=f$ for every $f \in \mathscr{F}_{\mathscr{L} / \mathcal{K}}(A)$ by definition. Since $\mathscr{L}$ topologically generates the algebra $\mathscr{L} \hat{\otimes}_{L^{\#}} A[[w]]$ over $\mathscr{K} \hat{\bigotimes}_{L^{*}} A[[w]]$ and since the two $\mathscr{K} \bar{\bigotimes}_{L^{\sharp}} A[[w]]$-automorphisms $f$ and $\left(f^{\prime}\right)_{*}$ coincide on $\mathscr{L}$, we have $\left(f^{\prime}\right)_{*}=f$ for every

$$
f \in \mathbf{I n f}-\operatorname{diff}-\operatorname{bir}(A) .
$$

We know that every element $f \in \mathscr{F}_{\mathscr{L} / \mathscr{K}}(A)$ is given by some $\Phi \in \Gamma_{n L^{*}}(A)$ :

$$
f\left(Y_{i}(w, t)\right)=Y_{\imath}(\Phi(w), t) \quad \text { for } \quad 1 \leq i \leq m
$$

Now if we take an element

$$
\Phi=\left(\varphi_{1}(w), \varphi_{2}(w), \ldots, \varphi_{n}(w)\right) \in \Gamma_{n L}{ }^{*}(A),
$$

then there exists an infinitesimal deformation that sends $Y_{i}(w, t)$ to $Y_{i}(\Phi(w), t)$ if and only if the following condition is satisfied:

$$
F\left(Y_{1}(\Phi, t), Y_{2}(\Phi, t), \ldots, Y_{m}(\Phi, t)\right)=0
$$

for every differential polynomial

$$
F\left(Z_{1}, Z_{2}, \ldots, Z_{m}\right)
$$

with coefficients in $\mathscr{K}$ with respect to the derivations $\{\partial / \partial w, \partial / \partial t\}$ such that

$$
F\left(Y_{1}, Y_{2}, \ldots, Y_{m}\right)=0 .
$$

We notice here that not only the $Z_{i}$ but also the partial derivatives $\partial^{\alpha+\beta} Z_{i}$ / $\partial w^{\alpha} \partial t^{\beta}$ are involved in the differential polynomial

$$
F\left(Z_{1}, Z_{2}, \ldots, Z_{m}\right) \text {. }
$$

Considering $\Phi$ as a set of unknown functions in $w$, we expand the right side of (5.9.1) as a power series in $t$ :

$$
F\left(Y_{1}(\Phi, t), Y_{2}(\Phi, t), \ldots, Y_{m}(\Phi, t)\right)=\sum_{k>>-\infty} F_{k}(w, \Phi) t^{k},
$$

where $F_{k}(w, \Phi) \in L^{\natural}[[w]]\{\{\Phi\}\}$ if we use the notation of $\S 1$. So we have proved that 


$$
\Phi=\left(\varphi_{1}(w), \varphi_{2}(w), \ldots, \varphi_{n}(w)\right) \in \Gamma_{n L^{\prime}}(A)
$$

is in

\section{Inf-diff-bir ${ }_{K} L(A)$}

if and only if $F_{k}(w, \Phi)=0$ for every integer $k$ and every differential polynomial $F$ with coefficients in $\mathscr{K}$ such that $F\left(Y_{1}, Y_{2}, \ldots, Y_{m}\right)=0$. To complete the proof of the lemma, we have to show

$$
f_{(\Psi \circ \Phi) *}=f_{\Psi *} f_{\Phi *}
$$

for any two such transformations $\Phi, \Psi \in \Gamma_{n \Gamma^{\prime}}(A)$. In fact writing $\Phi=\mathrm{Id}+\Phi^{\prime}$, we have

$$
Y_{i}(\Phi(w), t)=Y_{i}\left(\mathrm{Id}+\Phi^{\prime}, t\right)=\sum_{l \in \mathbf{N}^{n}} \frac{1}{l !} \frac{\partial^{l} Y_{i}}{\partial w^{l}} \Phi^{\prime l}
$$

and so

$$
f_{\Phi *}\left(Y_{i}\right)=\sum_{l \in \mathbf{N}^{n}} \frac{1}{l !} \frac{\partial^{l} Y_{l}}{\partial w^{l}} \otimes \Phi^{\prime l}
$$

by definition. Hence

$$
f_{\Psi^{*}}\left(f_{\Phi^{*}}\left(Y_{\imath}\right)\right)=f_{\Psi *}\left(\sum_{l \in \mathbf{N}^{n}} \frac{1}{l !} \frac{\partial^{l} Y_{\imath}}{\partial w^{l}} \otimes \Phi^{\prime l}\right)
$$

since $f_{\Psi^{*}}$ is continuous

$$
=\sum_{l \in \mathbf{N}^{n}} \frac{1}{l !} f_{\Psi *}\left(\frac{\partial^{l} Y_{l}}{\partial w^{l}}\right) \otimes \Phi^{\prime l}
$$

since $f_{\Psi *}$ is a differential morphism, we have $f_{\Psi *}\left(\partial^{l} Y_{i} / \partial w^{l}\right)=\partial^{l} Y_{i}(\Psi(w), t) / \partial w^{l}$

$$
=\sum_{l \in \mathbf{N}^{n}} \frac{1}{l !} \frac{\partial^{l} Y_{i}(\Psi(w), t)}{\partial w^{l}} \otimes \Phi^{\prime l}
$$

by the Taylor formula

$$
\begin{aligned}
& =\sum_{l \in \mathbf{N}^{n}} \frac{1}{l !} \frac{\partial^{l} Y_{i}(\Psi(\Phi(w)), t)}{\partial w^{l}} \\
& =f_{(\Psi \circ \Phi) *}\left(Y_{i}\right)
\end{aligned}
$$

as wanted. So the lemma is proved.

Let $A$ be an $L^{\natural}$-algebra, $f \in \mathscr{F}_{\mathscr{L} / \mathscr{K}}$ and 


$$
h \in \mathbf{I n f}-\operatorname{diff}-\mathbf{b i r}_{K} L(A) .
$$

So

$$
f: \mathscr{L} \rightarrow A[[w, t]]\left[t^{-1}\right]
$$

is a differential $\mathscr{K}$-algebra morphism and $h$ is a $\mathscr{K} \widehat{\bigotimes}_{L^{*}} A[[w]]$-automorphism of the differential algebra

$$
\mathscr{L} \bar{\bigotimes}_{L^{\#}} A[[w]]
$$

We denote by $h f$ the composite morphism

$$
\mathscr{L} \rightarrow \mathscr{L} \bar{\otimes}_{L^{\sharp}} A[[w]] \stackrel{h}{\rightarrow} \mathscr{L} \bar{\otimes}_{L^{\sharp}} A[[w]] \stackrel{\sim}{\rightarrow} \overline{\mathbf{Q}[f(\mathscr{L}), A[[w]]]} \subset A[[w, t]]\left[t^{-1}\right],
$$

where the first morphism is the canonical morphism and the third is the isomorphism of Lemma (5.6). Then $h f \in \mathscr{F}_{\mathscr{L} / \mathscr{K}}(A)$. Hence

$$
\text { Inf-diff- } \text { bir }_{K} L(A) \times \mathscr{F}_{\mathscr{L} / \mathcal{K}}(A) \rightarrow \mathscr{F}_{\mathscr{L} / \mathscr{K}}(A) \quad(h, f) \mapsto h f
$$

is an operation of the group functor Inf-diff-bir ${ }_{K} L$ on the functor $\mathscr{F}_{\mathscr{L} / \mathcal{K}}$.

THEOREм (5.10). The Lie-Ritt functor Inf-diff-bir ${ }_{K} L$ operates on the functor $\mathscr{F}_{\mathscr{L} / \mathscr{K}}$ and (Inf-diff-bir $\left.{ }_{K} L, \mathscr{F}_{\mathscr{L} / \mathscr{K}}\right)$ is a principal homogeneous space.

Proof. We have seen above the first assertion. So it remains to prove the second. Let $i: \mathscr{L} \rightarrow A[[w, t]]\left[t^{-1}\right]$ be the canonical morphism. If we consider the morphism

$$
k: \operatorname{Inf}-\operatorname{diff}-\operatorname{bir}_{K} L(A) \rightarrow \mathscr{F}_{\mathscr{L} / \mathcal{K}}(A) f \mapsto f i,
$$

then $k$ is injective by Lemma (5.6) and since $h_{*} i=h$ for any $h \in \mathscr{F}_{\mathscr{L} / \mathcal{K}}(A)$, surjective.

We have seen in $\S 4$ that every result for the infinitesimal deformation functor $\mathscr{F}_{\mathscr{L} / \mathscr{K}}$ of the canonical morphism $\mathscr{L} \rightarrow L^{\natural}[[w, t]]\left[t^{-1}\right]$ holds for the infinitesimal deformation functor $\mathfrak{F}_{\mathscr{L} / \mathcal{K}}$ of the universal Taylor morphism $\mathscr{L} \rightarrow \mathscr{L}^{\natural}[[w, T]]$ except for the surjectivity of the morphism (4.22).

THEOREM (5.11). The restriction

$$
\left(\text { Inf-diff-bir }{ }_{K} L\right) \bigotimes_{L}, \mathscr{L}^{\natural}
$$

of the Lie-Ritt functor Inf-diff-bir ${ }_{K} L$ on the category $\left(A \lg / \mathscr{L}^{\natural}\right)$ operates on the functor $\mathfrak{F}_{\mathscr{L} / \mathcal{K}}$ and $\left(\mathbf{I n f}\right.$-diff-bir $\left.{ }_{K} L, \mathfrak{F}_{\mathscr{L} / \mathscr{K}}\right)$ is a principal homogeneous space. 
Proof. We notice that if we replace the canonical morphism $\mathscr{L} \rightarrow L^{\natural}[[w$, $t]]\left[t^{-1}\right]$ by the universal Taylor morphism $\mathscr{L} \rightarrow \mathscr{L}^{\natural}[[w, T]]$, then Lemmas $(5.1)$ (5.3), (5.5), (5.6), (5.7) and Corollaries (5.2), (5.3) hold. Now the theorem follows from the proof of Theorem (5.10).

COROLlary (5.12). The functorial morphism (4.22) is surjective for all $\mathscr{L}^{\natural}$ algebra $A$.

Proof. The Corollary follows from Theorems (5.10), (5.11) and Proposition (4.16).

We had better introduce a new notation to indicate the infinitesimal Galois group.

Definition (5.13). We call the Lie-Ritt functor Inf-diff-bir ${ }_{K} L$ the infinitesimal Galois group of the ordinary differential field extension $L / K$ and we denote it by $\operatorname{Inf}-\mathbf{g a l}(L / K)$.

THEOREM (5.14). Let $L / K$ be an ordinary differential field extension satisfying the condition (F.C) and $L \supset M \supset K$ be a differential intermediate field. Then there exists canonical morphism

$$
\operatorname{Inf}-\operatorname{gal}(L / K) \rightarrow \operatorname{Inf}-\operatorname{gal}(M / K) \bigotimes_{M}, L^{\natural}
$$

such that

$$
\operatorname{Inf}-\operatorname{gal}(L / K)(A) \rightarrow \text { Inf-gal }(M / K) \bigotimes_{M}, L^{\natural}(A)=\mathbf{I n f}-\operatorname{gal}(L / K)(A)
$$

is surjective for every $\mathscr{L}^{\natural}$-algebra $A$.

Proof. The theorem follows from Proposition (4.16).

If $L / K$ is a strongly normal extension with Galois group $G$, what is the infinitesimal Galois group $\operatorname{Inf}-\operatorname{gal}(L / K)$ ? The answer is that $\operatorname{Inf}-\operatorname{gal}(L / K)$ is almost $G$.

THEOREM (5.15). If $L / K$ is a strongly normal extension with Galois group $G$, then we have

$$
\operatorname{Inf}-\operatorname{gal}(L / K) \simeq \hat{G}_{L^{4}} .
$$

Here $\hat{G}$ denotes the formal group associated with algebraic group scheme $G$. 
Proof. We denote the field $C_{\mathscr{K}}$ of constants of the differential field $\mathscr{K}$ by $\mathscr{C}$. It follows from Theorem (3.14) that the extension $\mathscr{L} / \mathscr{K}$ is a strongly normal extension with Galois group $G_{\mathscr{C}}$. So by Theorems (2.13), (3.10) in [U3] generalized to partial case, there exists a model $\mathscr{X}$ of the differential field extension $\mathscr{L} / \mathscr{K}$ such that $G_{\mathscr{K}}$ operates on $\mathscr{X}$ in such a way that

$$
G_{\mathscr{C}} \times_{\mathscr{C}} \mathscr{X}=G_{\mathscr{K}} \times_{\mathscr{K}} \mathscr{X} \rightarrow \mathscr{X} \times_{\mathscr{K}} \mathscr{X} \quad(g, x) \mapsto(g x, x)
$$

is an isomorphism of schemes with derivations. In fact in the proof of Theorem (3.14), we constructed $\mathscr{X}$ as

$$
X \otimes_{K} Q\left(K \otimes_{C_{K}} L^{\#}\right)
$$

Let $A$ be an $L^{\natural}$-algebra and

$$
f \in \mathscr{F}_{\mathscr{L} / \mathscr{K}}(A)
$$

be an infinitesimal deformation of the canonical morphism

$$
\mathscr{L} \rightarrow L^{\natural}[[w, t]]\left[t^{-1}\right]
$$

so that $f: \mathscr{L} \rightarrow A[[w, t]]\left[t^{-1}\right]$ is a morphism of differential algebras. The morphism $f$ defines a differential morphism

$$
\tilde{f}: \mathscr{L} \otimes_{L^{\#}} A[[w]] \rightarrow A[[w, t]]\left[t^{-1}\right] \quad(a \otimes b \mapsto f(a) b)
$$

and consequently a morphism

$$
\tilde{f}^{a}: \operatorname{Spec} A[[w, t]]\left[t^{-1}\right] \rightarrow \mathscr{X} \bigotimes_{L^{\#}} A[[w]]=\mathscr{X} \bigotimes_{\mathscr{K}}\left(\mathscr{K} \otimes_{L^{\#}} A[[w]]\right)
$$

of schemes with derivations. In particular if we take the trivial deformation

$$
\iota: \mathscr{L} \rightarrow L^{\natural}[[w, t]]\left[t^{-1}\right] \hookrightarrow A[[w, t]]\left[t^{-1}\right]
$$

as $f$, then we get

$$
\tau: \mathscr{L} \otimes_{L^{\sharp}} A[[w]] \rightarrow A[[w, t]]\left[t^{-1}\right]
$$

and

$$
\tilde{\iota}^{a}: \operatorname{Spec} A[[w, t]]\left[t^{-1}\right] \rightarrow \mathscr{X} \otimes_{L^{\#}} A[[w]] .
$$

Now we denoting $\mathscr{K} \bigotimes_{L^{\#}} A[[w]]$ by $\mathscr{B}$ so that

$$
\mathscr{X} \otimes_{L^{\sharp}} A[[w]]=\mathscr{X} \otimes_{\mathscr{K}}\left(\mathscr{K} \bigotimes_{L^{\#}} A[[w]]\right)=\mathscr{X}_{\mathscr{B}},
$$

the morphism 


$$
\begin{aligned}
\left(\tilde{f}^{a}, \tilde{c}^{a}\right): \operatorname{Spec} A[[w, t]]\left[t^{-1}\right] & \rightarrow X_{\mathscr{B}} \times_{\mathscr{B}} \mathscr{X}_{\mathscr{B}} \\
& \simeq G_{\mathscr{B}} \times_{\mathscr{B}} X_{\mathscr{B}} \\
& =G_{\mathscr{C}} \times_{\mathscr{C}} \mathscr{X}_{\mathscr{B}}
\end{aligned}
$$

composed with the projection

$$
G_{\mathscr{C}} \times_{\mathscr{C}} \mathscr{X}_{\mathscr{B}} \rightarrow G_{\mathscr{C}}
$$

gives an $A[[w, t]]\left[t^{-1}\right]$-valued point

$$
g_{0}: \operatorname{Spec} A[[w, t]]\left[t^{-1}\right] \rightarrow G_{\mathscr{C}},
$$

which is a morphism of schemes with derivations. So since $G_{\mathscr{C}}$ is a scheme with the trivial derivation, the $A[[w, t]]\left[t^{-1}\right]$-valued point $g_{0}$ factors through an $A$-valued point

$$
g: \operatorname{Spec} A \rightarrow G_{\mathscr{C}} .
$$

Since $f$ is an infinitesimal deformation of the canonical morphism, the morphism $g_{0}$ is, modulo $N(A)[[w, t]]\left[t^{-1}\right]$, congruent to the constant morphism

$$
\operatorname{Spec} A[[w, t]]\left[t^{-1}\right] \rightarrow G_{\mathscr{C}}
$$

that maps $\operatorname{Spec} A[[w, t]]\left[t^{-1}\right]$ to 1 of $G_{\mathscr{C}}$. Namely the composite morphism

$\operatorname{Spec} A[[w, t]]\left[t^{-1}\right] / N(A)[[w, t]]\left[t^{-1}\right] \rightarrow \operatorname{Spec} A[[w, t]]\left[t^{-1}\right] \rightarrow G_{\mathscr{C}}$

of the natural morphism and $g_{0}$ is the morphism that maps

$$
\text { Spec } A[[w, t]]\left[t^{-1}\right] / N(A)[[w, t]]\left[t^{-1}\right]
$$

to 1 . So $g$ is also, modulo $N(A)$, congruent to the constant morphism $\operatorname{Spec} A \rightarrow$ $G_{\mathscr{C}}$ that maps Spec $A$ to 1 of $G_{\mathscr{C}}$. We notice here the ring extensions

$$
\mathscr{K} \otimes_{L^{*}} A[[w]] \supset \mathscr{K} \bigotimes_{\mathscr{C}} A \supset A .
$$

Since $G$ operates on $\mathscr{X}$, the $A$-valued point $g$ defines a $\mathscr{K} \bigotimes_{\mathscr{C}} A$-automorphism $\varphi_{g}$ of the scheme

$$
\mathscr{X}_{\mathscr{K}} \otimes_{\mathscr{K}}\left(\mathscr{K} \otimes_{\mathscr{C}} A\right)=\mathscr{X}_{\mathscr{K} \otimes_{\mathscr{G}} A}
$$

of scheme with derivations:

$$
\varphi_{g}: \mathscr{X}_{\mathscr{K} \otimes_{\mathscr{G}} A} \rightarrow \mathscr{X}_{\mathscr{K} \otimes_{\mathscr{G} A}} .
$$

By base change, we get an automorphism

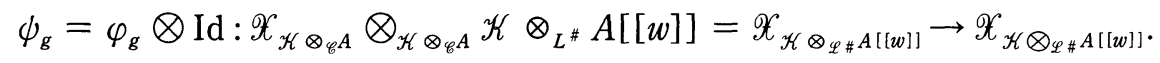


It follows from the construction of $\phi_{g}$ that we have

$$
\phi_{g}^{\circ} \tilde{\iota}^{a}=\tilde{f}^{a} .
$$

Since the reduction $\varphi_{g} \otimes_{A} A / N(A)$ of $\varphi_{g}$ is the identity automorphism of $\mathscr{X}_{\mathscr{K} \otimes_{\mathscr{q} A} A}$, the reduction $\phi_{g} \otimes_{A} A / N(A)$ is the identity automorphism of $\mathscr{X}_{\mathscr{K}^{*} \otimes_{\left.L^{*} A[w]\right]}} \bigotimes_{A}$ $A / N(A)$. Let $\operatorname{Spec} \mathscr{R}$ be an affine open set of the $\mathscr{K}$-scheme $\mathscr{X}$. So $\mathscr{R}$ is a subring of $\mathscr{L}$ such that the quotient field $Q(\mathscr{R})$ is $\mathscr{L}$ and closed under the set of derivations. Since

$$
\phi_{g} \otimes_{A} A / N(A)
$$

is the identity and since

$$
\mathscr{X}_{\mathscr{K} \otimes_{L \# A[\{w]]}} \otimes_{A} A / N(A)
$$

is homeomorphic to

$$
\mathscr{X}_{\mathcal{K} \otimes_{L} \# A[[w]]} \otimes_{A} A / N(A),
$$

the automorphism $\phi_{g}$ leaves the open set

$$
\operatorname{Spec} \mathscr{R} \otimes_{\mathscr{K}}\left(\mathscr{K} \bigotimes_{L^{\sharp}} A[[w]]\right)=\operatorname{Spec} \mathscr{R} \bigotimes_{L^{\sharp}} A[[w]]
$$

of $\mathscr{X}_{\mathscr{B}}$ invariant. For $v \in \mathscr{R}$, we have

$$
\phi_{g}^{*}(v)=v+\bar{v} \in \mathscr{R} \otimes_{L^{\#}} A[[w]]
$$

with nilpotent $\bar{v}$. In particular if $0 \neq v \in \mathscr{R}$, then

$$
\phi_{g}^{*}(v)=v+\bar{v}=v\left(1+v^{-1} \bar{v}\right)
$$

is invertible and is an element of $\mathscr{L} \bigotimes_{L^{*}} A[[w]]$. Thus we get a $\mathscr{B}$-isomorphism

$$
\phi_{g}^{*}: \mathscr{L} \bigotimes_{L^{\#}} A[[w]] \rightarrow \mathscr{L} \bigotimes_{L^{\#}} A[[w]]
$$

such that $\tilde{\iota}=\phi_{g}^{*} \circ \tilde{f}$. We have thus proved that $\mathscr{F}_{\mathscr{L} / \mathcal{K}}(A)$ is a principal homogeneous space of

$$
\hat{G}_{\mathscr{C}}(A)=\left\{f \in G_{\mathscr{C}}(A) \mid f \equiv 1 \bmod N(A)\right\} .
$$

So the functor $\mathscr{F}_{\mathscr{L} / \mathscr{H}}$ is a principal homogeneous space of $\hat{G}_{\mathscr{C}}$ restricted on the category $\left(A \lg / L^{\natural}\right)$, which is nothing but $\hat{G}_{L^{*}}$. The theorem now follows if we notice that the $\mathscr{K} \otimes_{L^{\sharp}} A[[w]]$-automorphism

$$
\phi_{g}^{*}: \mathscr{L} \bigotimes_{L^{\#}} A[[w]] \rightarrow \mathscr{L} \bigotimes_{L^{\#}} A[[w]]
$$

can be extended uniquely to a continuous $\mathcal{K} \otimes_{L^{*}} A[[w]]$-automorphism of the 
completion $\mathscr{L} \hat{\bigotimes}_{L^{*}} A[[w]]$.

The invariant Inf-gal $(L / K)$ ignores algebraic extensions and extensions generated by constants.

THEOREM (5.16). Let $L / K$ be an ordinary differential field extension satisfying the condition (F.C) in $\S 3$ and $L \supset M \supset K$ be a differential intermediate field.

(1) If the field $L$ is algebraic over $M$, then $\mathbf{I n f}-\mathbf{g a l}(L / K)$ is canonical isomorphic to Inf-gal $(L / K) \bigotimes_{M}, L^{\natural}$.

(2) If the field $L$ is generated by constants over $M$, then Inf-gal $(L / K)$ is canonically isomorphic to $\mathbf{I n f - g a l}(L / K) \bigotimes_{M}, L^{\natural}$.

Proof. The canonical morphism

$$
\operatorname{Inf}-\operatorname{gal}(L / K) \rightarrow \operatorname{Inf}-\operatorname{gal}(L / K) \bigotimes_{M^{*}} L^{\natural}
$$

is surjective by Theorem (5.14). It follows from the construction of $\mathscr{L}$ and $\mathscr{K}$ that if the field $L$ is algebraic over $K$, then the field $\mathscr{L}_{L / K}$ is algebraic over $\mathscr{L}_{M / K}$. So by the proof of Lemma (4.5), the morphism (5.17) is injective. This proves the first assertion of the theorem. Let us now assume that the field $L$ is generated by constants over $M$. The field of constants of the differential subfield $\mathscr{L}_{M / K}$ of the ordinary differential field $\left(M^{\natural}[[t]]\left[t^{-1}\right], d / d t\right)$ is $M^{\natural}$. Similarly the field of constants of the differential subfield $\mathscr{L}_{L / K}$ of the ordinary differential field $\left(L^{\natural}[[t]]\left[t^{-1}\right]\right.$, $d / d t)$ is $L^{\natural}$. We consider $\left(M^{\natural}[[t]]\left[t^{-1}\right], d / d t\right)$ as a differential subfield of ( $\left.L^{\natural}[[t]]\left[t^{-1}\right], d / d t\right)$ and we apply Lemma $(1.1)$ to $\mathscr{L}_{L / K}$ and $\mathscr{L}_{M / K}$ so that $\mathscr{L}_{M / K}$ and $L^{\natural}$ are linearly disjoint over $M^{\natural}$. Hence

$$
\mathscr{L}_{M} / K \cdot L^{\natural} \simeq \mathbf{Q}\left(\mathscr{L}_{M / K} \otimes_{M} L^{\natural}\right) .
$$

Since the field $L$ is generated by constants over $M$, the subfield $\mathscr{L}_{M / K} \cdot L^{\#}$ of the differential field $L^{\#}[[t]]\left[t^{-1}\right]$ contains $i(L)$. As the subfield $\mathscr{L}_{M / K} \cdot L^{\#}$ of the differential field $L^{\#}[[t]]\left[t^{-1}\right]$ is closed under the set of derivations and contains $i(L)$, we have

$$
\begin{gathered}
\mathscr{L}_{M / K} \cdot L^{\#}=\mathscr{L}_{L / K} . \\
\mathscr{L}_{L / K} \simeq \mathbf{Q}\left(\mathscr{L}_{M / K} \otimes_{M^{\#}} L^{\#}\right) .
\end{gathered}
$$

Similarly we have

$$
\mathscr{K}_{L / K} \simeq \mathbf{Q}\left(\mathscr{K}_{M / K} \otimes_{M^{\#}} L^{\#}\right) .
$$

It follows in particular from (5.20) and (5.21) that the field $\mathscr{L}_{L / K}$ is generated over 
$\mathscr{K}_{L / K}$ by the canonical image of $\mathscr{L}_{M / K}$. So the canonical morphism (5.18) is injective and the second assertion of the theorem is proved.

COROLlary (5.22). Let $L / K$ be an ordinary differential field extension satisfying the condition (F.C) in $\S 3$.

(1) If the field $L$ is algebraic over $K$, then we have Inf-gal $(L / K)=0$.

(2) If the field $L$ is generated by constants over $K$ by constants, then we have $\operatorname{Inf}-\operatorname{gal}(L / K)=0$.

Proof. The corollary is a particular case of the theorem where the intermediate field $M$ coincides with $K$.

\section{REFERENCES}

[D] Drach, J., Essai sur une théorie générale de l'intégation et sur la classification des transcendentes, Annales sci. de l'École normale Sup., (3) 15 (1898), $243-384$.

[K] Kolchin, E., Differential algebra and algebraic groups, Accademic Press, New York and London, 1973.

[L] Lang, S., Algebra, Addison-Wesley, Reading, Massachusetts. Menlo Park, California $\cdot$ London $\cdot$ Sydney $\cdot$ Manila, 1971.

[N] Nichols, W. and Weisfeiler, B., Differential formal group of J. F. Ritt, Amer. J. Math., 104 (1982), 943-1003.

[P] Pommaret, J. F., Differential Galois theory, Gordon and Breach, New York, London, Paris, 1983.

[R] Ritt, J. F., Associative differential operations, Ann. Math., 51 (1950), 756 765.

[S] Serre, J.-P., Lie algebras and Lie groups, Benjamin, New York, 1965.

[S.G.A.D] Demazure, M. et Grothendieck, A., Schemas en groupes I, Lecture Notes in Math., vol. 151, Springer-Verlag, Berlin-Heidelberg-New York, 1970.

[U1] Umemura, H., On the irreducibility of the first differential equation of Painlevé, Algebraic geometry and commutative algebra in honor of Masayoshi NAGATA, Kinokuniya, Tokyo, 1987, pp. 771-789.

[U2] - On a class of numbers generated by differential equations related with algebraic groups, Nagoya Math. J., 133 (1994), 1-55.

[U3] - Galois Theory of algebraic and differential equations, Nagoya Math. J., 144 (1996), 1-58.

[V1] Vessiot, E., Sur la théorie de Galois et ses diverses généralisations, Annales sci. de l'École normale Sup., (3) 21 (1904), 9-85.

[V2] - Sur une généralisation de la réductibilité des équations et systémes d'équations finies ou différentielles, Annales sci. de l'École normale Sup., (3) 63 (1946), 1-22.

[W] Weisfeiler, On Lie algebras of differential formal groups of Ritt, Bull. Amer. Math. Soc., 84 (1978), 127-130. 
Graduate School of Polymathematics

Nagoya University

Chikusa-ku, Nagoya 464-01

Japan

email: umemura@math.nagoya-u.ac.jp 